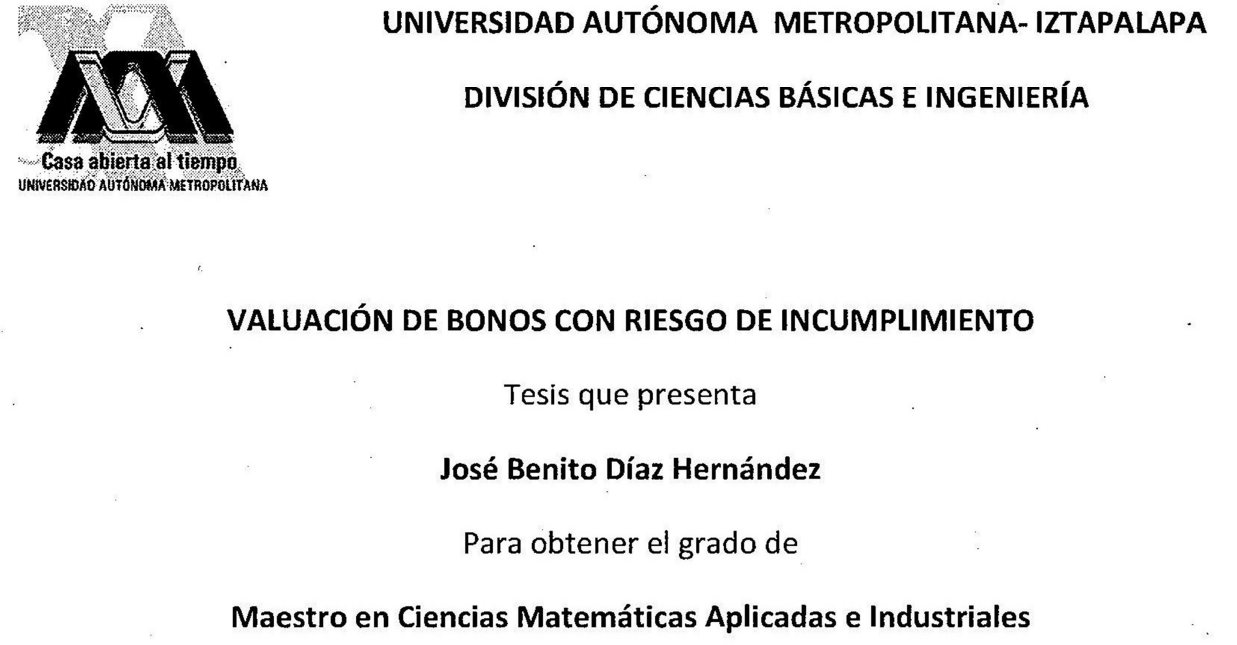

Asesora: Dra. Patricia Saavedra Barrera.

Jurado Calificador:

Presidente: Dr. Juan Ruiz de Chávez Somoza UAM-I

Secretario: Dra. Patricia Saavedra Barrera

UAM-I Patricia Saantediay

Vocal: Dr. Netzahualcóyotl Castañeda Leyva. UG 
UNIVERSIDAD AUTÓNOMA METROPOLITANA- IZTAPALAPA DIVISIÓN DE CIENCIAS BÁSICAS E INGENIERÍA

\title{
VALUACIÓN DE BONOS CON RIESGO DE INCUMPLIMIENTO
}

\author{
Tesis que presenta \\ José Benito Díaz Hernández \\ Para obtener el grado de \\ Maestro en Ciencias Matemáticas Aplicadas e Industriales
}

Asesora: Dra. Patricia Saavedra Barrera.

Jurado Calificador:

Presidente: Dr. Juan Ruiz de Chávez Somoza UAM-I

Secretario: Dra. Patricia Saavedra Barrera UAM-I

Vocal: Dr. Netzahualcóyotl Castañeda Leyva. UG

México, D.F. febrero 2013. 


\section{Agradecimientos}

Este trabajo fue posible gracias al apoyo y enseñanzas de mi asesora de tesis, la Dra. Patricia Saavedra Barrera. Gracias por su tiempo y revisión de este tesis.

A mis sinodales, el Dr. Juan Ruiz de Chávez Somoza y el Dr. Netzahualcóyotl Castañeda Leyva, por sus valiosos comentarios, sugerencias y correcciones para enriquecer este trabajo.

A mis padres por su guía, sus bendiciones y enseñanzas, por compartir conmigo sus experiencias y haberme apoyado siempre, y sobre todo por su amor incondicional.

A mi hermano y hermana por su compañía, por todas las buenas experiencias que hemos vivido a lo largo de nuestras vidas y por guiarme en muchos momentos.

A Naye por estar a mi lado durante estos últimos años y dejarme pasar momentos inolvidables a su lado. Gracias por sus comentarios y observaciones en mi trabajo. Este es uno de nuestros triunfos y sé que celebraremos más.

Así mismo, quiero agradecer a la UAM y al ICyTDF por su apoyo otorgado para la culminación de este trabajo.

Finalmente, agradezco a todas aquellas personas que compartieron momentos durante la realización de este trabajo. 


\section{Índice general}

1 Antecedentes 1

1.1 Modelo de Black \& Scholes . . . . . . . . . . . . . . . . 1

1.1.1 Modelo de Black \& Scholes . . . . . . . . . . . . . . . 3

1.2 Ecuación diferencial parcial . . . . . . . . . . . . . . 7

1.3 Opciones con barrera . . . . . . . . . . . . . . . . . 13

1.4 Modelos de tasa de interés . . . . . . . . . . . . . . . . 18

1.4.1 Modelo de Merton (1973) . . . . . . . . . . . . . 21

1.4.2 Modelo de Vasicek (1977) . . . . . . . . . . . . . . . 23

1.4.3 Modelo de Cox, Ingersoll, Ross (1985) . . . . . . . . . . . . . . . 25

1.5 Estimación de parámetros para modelos de tasa de interés . . . . . . . . 27

1.5.1 Modelo Vasicek . . . . . . . . . . . . . . . . . 27

1.5 .2 Modelo CIR . . . . . . . . . . . . . . . . . . 30

2 Valuación de bonos corporativos $\quad 33$

2.1 Modelo estructural de Merton . . . . . . . . . . . . . . . 33

2.2 Modelo de Merton con tasa de interés estocástica . . . . . . . . . . . . . . 38

2.2.1 Modelo de Merton bajo el modelo de Vasicek . . . . . . . . . . . . . 38

2.2.2 Modelo de Merton bajo el modelo de CIR . . . . . . . . . . . . . . 41

2.3 Modelo de Black y Cox . . . . . . . . . . . . . . . . . 44 
3 Modelos de Intensidad

3.1 Conjuntos de información . . . . . . . . . . . . . . 50

3.2 Ejemplos de modelos de intensidad . . . . . . . . . . . . . . 51

3.2 .1 Procesos Poisson . . . . . . . . . . . . . . . . 52

3.2.2 Procesos Poisson no homogéneos . . . . . . . . . . . . . 53

3.2 .3 Procesos Cox . . . . . . . . . . . . . . . . 55

3.3 Valuación de bonos con incumplimiento . . . . . . . . . . . . . . 59

3.3.1 Valuación bajo dependencia entre $r_{t} \mathrm{y} \lambda_{t} \ldots \ldots \ldots 61$

4 Aplicación de los modelos para valuar bonos corporativos de empresas mexicanas

4.1 Modelo estructural de Merton . . . . . . . . . . . . . . . . . 64

4.2 Modelo de Merton con tasa de interés estocástica . . . . . . . . . . . . . 67

4.3 Modelo de Black y Cox . . . . . . . . . . . . . . 71

4.4 Modelos de Intensidad . . . . . . . . . . . . . . . . . . . . 74

5 Conclusiones $\quad \mathbf{8 0}$

$\begin{array}{lll}\text { A Algunos resultados de probabilidad } & 83\end{array}$

A.1 Teorema de Girsanov . . . . . . . . . . . . . . . . . . . 83

A.2 Teoremas fundamentales de valuación de activos. . . . . . . . . . . . 87

$\begin{array}{lll}\text { B Algunos resultados de movimientos brownianos } & \mathbf{8 8}\end{array}$ 


\section{Introducción}

En los mercados financieros, en particular en el mercado de dinero, se muestra que el precio a que se negocian los bonos corporativos es superior al precio de los bonos gubernamentales. Este exceso es conocido como el diferencial de rentabilidad o spread y, se debe, generalmente al riesgo de insolvencia al que se ve expuesto quien adquiere los bonos emitidos por entidades privadas. Dentro de este contexto, definimos el riesgo de insolvencia o de crédito, como el riesgo derivado del incumplimiento de los comprominisos por parte del emisor del bono.

A diferencia del riesgo de mercado, el desarrollo de metodologías para medir el riesgo de crédito ha sido relativamente menor, ya que las dificultades para la identificación y medición de los factores que intervienen en este tipo de riesgo han sido mayores, por ejemplo, el fenómeno de la información asimétrica, el riesgo moral, la ausencia de una teoría de la quiebra empresarial y la ausencia de información adecuada, entre otros. Aún con estos obstáculos se han creado diferentes metodologías que han sido aplicadas con el propósito de estimar la probabilidad de incumplimiento. Entre estas podemos mencionar en general técnicas estadísticas, multivariadas, análisis de modelos de clasificación, árboles de decisión, modelos de elección cualitativa y el análisis de matrices de transición entre otros.

El pionero en la investigación y modelación de predicción de la insolvencia empresarial fue, Ewdard I. Altman, prestigiado académico de la escuela de negocios de Nueva York, conocido por el desarrollo del Z-score para predecir la bancarrota publicado en 1968. Mencionó que el próximo gran reto financiero sería la administración de riesgos de crédito, que consiste en medir, minimizar y prevenir las pérdidas esperadas y las pérdidas no esperadas que surgen de las actividades relacionadas con el otorgamiento de préstamos en general. 
Desde un punto de vista académico, los modelos de riesgo de crédito se clasifican principalmente en modelos estructurales, modelos de forma reducida (modelos de intensidad) y modelos actuariales. En este trabajo nos enfocamos en el estudio de los modelos estructurales y los modelos de intensidad con el objetivo de modelar la deuda de las empresas, así como estimar la probabilidad de incumplimiento en el pago de su deuda a corto plazo.

El planteamiento básico de los modelos estructurales fue desarrollado por Merton (1973), en el cual propone valuar tanto el patrimonio como la deuda de una empresa con base teoría de valuación de opciones. El evento de no pago de la deuda se daría si, a su vencimiento, el valor de la misma supera el de los activos de la empresa, por lo cual los socios no ejercerían la opción de reclamar el valor de dichos activos en favor de los acreedores.

Sin embargo, la simplicidad del planteamiento muestra su escasa capacidad para explicar la situación real de las empresas y mercados financieros actuales, entre las principales razones se encuentra que se asume una tasa de interés constante y que toda la estructura de deuda de la empresa esta representada por un único bono cupón cero, lo que implica además que la insolvencia solo puede producirse en un único momento, es decir, en la fecha de vencimiento de la deuda. Una modificación del modelo clásico de Merton fue la introducción de los modelos de un sólo factor para modelar la tasa de interés a corto plazo, entre los más utlizados se encuentra el modelo de Vasicek y el modelo CIR.

Con el fin de intentar solucionar las limitaciones del modelo clásico de Merton y el modelo de Merton con tasa de interés estocástica, Black y Cox (1975) presentan un modelo en el que el emisor puede incumplir sus obligaciones en cualquier momentos durante la vigencia del bono, introduciendo una barrera para los activos de la empresa. En el momento que el valor de los activos cruce la barrera se produce el incumplimiento. Por su parte, Brennan y Schwartz (1980) pretenden corregir el supuesto de la tasa de interés constante introduciendo tipos de interés estocásticos en el modelo de Black y Cox. Otras modificaciones fueron presentadas en Longstaff y Schwartz (1995), las cuales contemplan la posibilidad de que los acreedores puedan forzar la insolvencia de la empresa en cualquier momento anterior a la fecha de vencimiento. Finalmente, Zhou (1997) introduce un modelo con saltos con la finalidad de introducir insolvencias 
impredecibles a priori.

Sin embargo, todos los modelos antes mencionados mantienen algunas dificultades como la de obtener el valor de la empresa y la de determinar cuál es la barrera bajo la cual la empresa se considera incapaz de hacer frente a sus obligaciones. El hecho de que no sean capaces de adoptar la posibilidad de insolvencia de forma impredecible, hace que las probabilidades de incumplimiento a muy corto plazo sean prácticamente cero y con ello, el diferencial de rentabilidad también. Este hecho contrasta fuertemente con lo que se observa en el mercado.

Ante las limitaciones de los modelos estructurales, surge una nueva línea de modelos conocidos como los modelos en forma reducida o modelos de intensidad. La ventaja de estos modelos con respecto a los anteriores es que no es necesario conocer el valor de determinadas variables como el valor de los activos de la empresa o de su volatilidad. Además, se tiene en cuenta el orden de prelación de la deuda mediante la estimación de la tasa de recuperación que no es más que la cantidad que se espera recuperar en el caso de que el emisor incumpla sus compromisos.

La metodología básica consiste en aplicar los sistemas de valuación tradicionales introduciendo una corrección en la tasa de descuento aplicable mediante una prima por el riesgo adicional (tasa de incumplimiento). De este modo, la insolvencia del emisor se establece como un tiempo de paro de un proceso estocástico que recoge la probabilidad de insolvencia de la empresa condicionada a que este hecho no ha ocurrido ya anteriormente. La elección del proceso se realiza exógenamente y como consecuencia de ello, se ha criticado estos modelos porque, en ocasiones, en dicha modelación se realizan ciertos ajustes para obtener resultados deseados.

Entre ellos, cabe destacar Jarrow y Turnbull (1995), el cual presenta un modelo libre de oportunidades de arbitraje, basándose en estructuras de tipos y probabilidad de incumplimiento constantes. Lando (2004) amplía el anterior modelo permitiendo correlación entre el proceso de tipos de interés y la probabilidad de incumplimiento y Das y Tufano (1995) introducieron correlación entre la tasa de recuperación y los tipos de interés. Jarrow, Lando y Turnbull (1997) muestran cómo utilizar las probabilidades de la matriz de transición de calidad crediticia para obtener los precios de los bonos corporativos. Últimamente se han desarrollados modelos híbridos, en los cuales se in- 
tenta conjuntar los modelos estructurales y los modelos de intensidad para eliminar las limitaciones individuales de cada modelo.

\section{Objetivos del trabajo}

En el presente trabajo se tienen tres principales objetivos, el primero es el estudio de los modelos estructurales, tales como el modelo de Merton y Black-Cox para la valuación de la deuda de una empresa a corto plazo. El segundo es el estudio de los modelos de intensidad y sus diferencias con los modelos estructurales en la valuación de bonos corporativos. Finalmente, la aplicación de los modelos a la valuación de la deuda de empresas mexicanas con objeto de analizar las dificultades que se presentan en la implementación.

En el primer capítulo se presentan los antecedentes del modelo de Black \& Scholes para la valuación de opciones, así como algunos aspectos de ecuaciones diferenciales estócasticas para la modelación de la dinámica de algunos procesos tales como las tasa de interés. Del mismo modo, se presenta algunos resultados importantes en la valuación de opciones con barrera. Por último, se muestra los modelos clásico de un factor en tasas de interés tales como: el modelo de Merton, el modelo de Vasicek y el modelo CIR; y la estimación de los parámetros para cada modelo.

En el segundo y tercero capítulo se estudian de manera separada los modelos estructurales y los modelos de intensidad para la valuación de bonos corporativos. Dentro de los modelos estructurales estudiados se encuentra el modelo de Merton, el modelo de Merton con tasa de interés estocástica y el modelo de Black y Cox. Por su parte, en los modelos de intensidad se presenta una breve descripción de los principales resultados aplicados a la valuación de bonos corporativos.

Finalmente, en el capítulo cuatro se presentan los resultados obtenidos en la valuación de los bonos corporativos bajo los tres modelos estructurales y el modelo de intensidad, con objeto de modelar la deuda de las empresas (Cemex y Bimbo) a finales del 2012. Dentro de cada modelo, se pudo obtener la probabilidad de incumplimiento de la empresa, así como el cambio en el diferencial de rentabilidad o spread respecto al tiempo de maduración del bono. 


\section{Capítulo 1}

\section{Antecedentes}

El modelo de Black \& Scholes para la valuación de opciones es uno de los métodos más utilizados, ya que a pesar de sus conocidas limitaciones, es relativamente sencillo de entender y aplicar. En está sección nos limitaremos a estudiar el modelo de Merton para la valuación de bonos corporativos que es considerado el primer modelo estructural. Este modelo utiliza la teoría de valuación de opciones dada por el modelo de Black \& Scholes, por lo tanto también es conocido como modelo de Black-Scholes-Merton. A continuación se muestran los elementos necesarios para entender dicho modelo.

\subsection{Modelo de Black \& Scholes}

Un derivado es un activo cuyo valor esta determinado, de alguna manera, por el valor de otro activo o índice de referencia. Este último se denomina, generalmente, activo subyacente. Así, existe una importante relación entre el valor del instrumento que está siendo analizado y el valor del activo subyacente asociado con aquel instrumento.

Una opción es un contrato similar a un seguro, el pago de una prima da el derecho más no la obligación de ejercer el contrato. Por el contrario, la contraparte, aquél que recibe la prima, tiene la obligación de cumplir con las especificaciones del contrato cuando el tenedor decida ejercer su derecho. Una opción tiene dos participantes, el emisor de la opción y quien adquire la opción al que llamaremos tenedor. El tenedor de una opción tipo call, tiene el derecho de comprar un activo en una fecha determinada 
en un precio determinado. Mientras que el tenedor de una opción tipo put, tiene el derecho de verder un activo en una fecha determinada con un precio determinado. Debido a esta desigualdad en los compromisos de cada parte, el emisor de la opción recibirá una suma de dinero de quien compra la opción para aceptar su obligación. Este precio es conocido como prima.

Una opción se puede negociar en el mercado secundario, por lo tanto es necesario determinar su valor $V_{t}$ para cada tiempo $t \in[0, T]$ donde $T$ es el tiempo de maduración de la opción. Por otro lado si la opción está dentro de un mercado organizado, la prima de la opción está determinada por el mercado. Como caso particular se tiene que la prima que se paga al adquirir la opción es igual al valor de la opción al tiempo $t=0$.

Existen diferentes tipos de opciones, en general se puede clasificar en dos grandes grupos: opciones vainilla (básicas) y opciones exóticas. Las opciones vainilla se pueden clasificar en opciones europeas y opciones americanas. Las opciones europeas sólo se pueden ejercer en el tiempo de maduración $T$, mientras que las opciones americanas se pueden ejercer para cualquier tiempo $t \in[0, T]$.

El precio strike es el precio de ejercicio al cual se pacta un contrato. Tanto el precio del ejercicio como la fecha de expiración son especificados en el contrato. Cuando el tomador compra (vende) el activo bajo dichas condiciones, se dice que ejerce la opción. Existen cuatro tipos de particiantes en los mercados de opciones:

1. Compradores de opciones de compra.

2. Vendedores (emisores) de opciones de compra.

3. Compradores de opciones de venta.

4. Vendedores (emisores) de opciones de venta.

Se considera que los compradores tienen "posiciones largas“ y los vendedores "posiciones cortas“. La venta o ejercicio de una opción también puede llamarse “emisión de la opción“. Para los propósitos de valuación, todas las transacciones que están involucradas con la opción son simplificadas. Así, el monto de dinero asociado con la transacción es visto en términos de la ganancia (Payoff). En el caso de ejercicio de un 
call, el Payoff o función de pago del tenedor corresponde a la diferencia entre el precio de mercado del activo que se esta comprando $S_{t}$ y el valor del precio de ejercicio $K$ que se esta pagando por el activo. Es obvio que, si la diferencia es un valor negativo, el tomador no ejercerá la opción de compra, y en esa circunstancia, la ganancia será cero. Por otro lado si $S_{T}$ es mayor que $K$ entonces se ejercerá la opción y se tendrá una ganacia de $S_{T}-K$. El valor final del contrato para el caso de un call $C_{T}$ está dado por:

$$
C_{T}=M a ́ x\left(S_{T}-K, 0\right)=\left(S_{T}-K\right)_{+} \text {. }
$$

En otro caso, si el individuo adquiere una opción put, ésta será ejercida si al tiempo $T$ el precio del subyacente $S_{T}$ es menor que el precio de ejercicio $K$ y por tanto se tendrá una ganancia de $K-S_{T}$. El valor del contrato para el put está dado por:

$$
P_{T}=\operatorname{Máx}\left(K-S_{T}, 0\right)=\left(K-S_{T}\right)_{+} \text {. }
$$

A la función de pago de los casos anteriores la denotaremos por $h\left(S_{T}\right)$. El modelo de Black \& Scholes permite encontrar el precio justo de la opción al tiempo $t=0$.

\subsubsection{Modelo de Black \& Scholes}

Para obtener la ecuación de Black \& Scholes es necesario realizar algunos supuestos:

- Las ventas en corto y en descubierto están permitidas.

- Existe posibilidad de negociación continua.

- No hay costos de transacción ni impuestos. Todos los activos son divisibles.

- No hay pago de dividendos durante la vida del derivado.

- No hay oportunidad de arbitraje.

- El tipo de interés libre de riesgo $r$ es constante y el mismo para cualquier madurez del activo. 
Algunos de estos supuestos pueden relajarse. Por ejemplo, se pueden considerar una volatilidad $\sigma$ y la tasa de interés $r$ como funciones que dependen del tiempo.

El modelo propuesto por Black \& Scholes para describir el comportamiento de los precios es un modelo a tiempo continuo conformado de un activo riesgoso (cuyo precio al instante $t$ es $S_{t}$ ) y un activo libre de riesgo (cuyo precio al instante $t$ es $S_{t}^{0}$ ). La evolución de precio del activo libre de riesgo sigue la siguiente ecuación diferencial ordinaria:

$$
\frac{d S_{t}^{0}}{d t}=r S_{t}^{0}, S_{0}^{0}=1
$$

donde $r$ una constante no negativa es la tasa libre de riesgo. Por lo tanto podemos notar que para $t \geq 0$ el valor de un peso es de $S_{t}^{0}=e^{r t}$.

Por otro lado, se asume que el comportamiento del precio de subyacente $S_{t}$ en el intervalo $[0, T]$ sigue la siguiente ecuación diferencial estocástica:

$$
d S_{t}=S_{t}\left(\mu d t+\sigma d W_{t}\right), S_{0}>0
$$

donde $\mu$ es la tendencia, $\sigma>0$ es la volatilidad y $\left(W_{t}\right)$ es un movimiento Browniano estándar. La solución a esta ecuación estocástica esta dada por:

$$
S_{t}=S_{0} e^{\left(\mu-\frac{\sigma^{2}}{2}\right) t+\sigma W_{t}}
$$

que es un movimiento browniano geométrico.

En general una cartera de inversión consiste en una cantidad $\phi_{t}^{0}$ invertida en el activo libre de riesgo y una cantidad $\phi_{t}$ invertida en el activo con riesgo. A la pareja $\left(\phi_{t}^{0}, \phi_{t}\right)_{0 \leq t \leq T}$ que toma valores en $\Re^{2}$ se le conoce como estrategia de inversión la cual es adaptada a la filtración natural $\mathscr{F}_{t}$ del movimiento Browniano. Denotaremos el valor del portafolio en el instante $t$ como:

$$
\Pi_{t}=\phi_{t}^{0} S_{t}^{0}+\phi_{t} S_{t}
$$


Definición 1 (Cartera autofinanciable). Sea $\Pi_{t}$ una cartera de inversión, entonces $\Pi_{t}$ es una cartera autofinanciable si

$$
d \Pi_{t}=\phi_{t}^{0} d S_{t}^{0}+\phi_{t} d S_{t}
$$

Una cartera es autofinanciable si los cambios en el valor de la cartera dependen únicamente de los cambios en el valor del activo libre de riesgo y del activo riesgoso.

Definición 2 (Probabilidad neutra al Riesgo). Sea $(\Omega, \mathscr{A}, P)$ un espacio de probabilidad. Una medida de probabilidad $P^{*}$ es neutral al riesgo si,

- $P$ y $P^{*}$ son probabilidades equivalentes, es decir, cada una de ellas es absolutamente continua respecto a la otra.

- Bajo $P^{*}$ los precios descontados $\widetilde{S_{t}}=e^{-r t} S_{t}$ son martingala ${ }^{1}$.

De la ecuación diferencial estocástica que satisface $S_{t}$ se tiene que los precios descontados del activo libre de riesgo $\widetilde{S_{t}}$ satisface la siguiente ecuación diferencial estocástica:

$$
\begin{aligned}
d \widetilde{S}_{t} & =-r e^{-r t} S_{t} d t+e^{-r t} d S_{t} \\
& =\widetilde{S}_{t}\left((\mu-r) d t+\sigma d W_{t}\right)
\end{aligned}
$$

Por lo tanto si $\widetilde{W_{t}}=W_{t}+(\mu-r) t / \sigma$ entonces,

$$
d \widetilde{S}_{t}=\widetilde{S}_{t} \sigma d \widetilde{W}_{t}
$$

Por el teorema de Girsanov ${ }^{2}$, tomando $\theta_{t}=(\mu-r) / \sigma$, el proceso $\widetilde{W}_{t}=W_{t}+(\mu-r) t / \sigma$ es un movimiento browniano estándar bajo la probabilidad $P^{*}$. Por lo tanto, el precio descontado del activo con riesgo es una martingala y esta dado por:

$$
S_{t}=S_{0} e^{\left(r-\frac{1}{2} \sigma^{2}\right) t+\sigma \widetilde{W}_{t}}
$$

\footnotetext{
${ }^{1} \widetilde{S}_{t}$ es martingala si cumple que $E^{*}\left[\widetilde{S}_{t+s} \mid \mathscr{F}_{t}\right]=\widetilde{S}_{t}$, para $s>0$.

${ }^{2}$ Ver Apéndice A, Teorema A.1.2
} 
Definición 3 (Arbitraje). Un arbitraje es una cartera de inversión que satisface $\Pi_{0}=$ 0 y que además satisface $P\left(\Pi_{t} \geq 0\right)=1$ y $P\left(\Pi_{t}>0\right)>0$ para algún tiempo $t>0$.

Por el Primer teorema fundamental de la Valuación de Activos (Ver Apéndice A.2), aseguramos la ausencia de oportunidad de arbitraje.

Definición 4 (Estrategia de inversión admisible). Una estrategia de inversión $\left(\phi_{t}^{0}, \phi_{t}\right)$ con $t \in[0, T]$ es admisible si es autofinanciable y si el valor descontado de la cartera $\widetilde{\Pi}_{t}$ es positivo para toda $t$ y cuadrado integrable bajo $P^{* 3}$.

Definición 5 (Opción replicable). Se dice que una opción es replicable si se puede construir una cartera admisible cuyo valor en cualquier tiempo $t \in[0, T]$ coincida con el valor de la opción.

Con la deficiones anteriores podemos introducir el siguiente teorema:

Teorema 1.1.1. En el modelo de Black \& Scholes, una opción definida por la variable aleatoria $h$, positiva, $\mathscr{F}_{T}$ medible y cuadrado integrable bajo la probabilidad $P^{*}$ es replicable y el valor al instante t del portafolio correspondiente está dado por

$$
\Pi_{t}=E^{*}\left[e^{-r(T-t)} h \mid \mathscr{F}_{t}\right]
$$

Demostración. Ver Lamberton y Lampeyre (2008) pág. 68-69.

Corolario 1.1.1. Si en particular h es la función de pago de un call o de un put europeo, al tiempo $t<T$, entonces el valor del call y del put están dados por:

$$
\begin{aligned}
& C\left(t, S_{t}\right)=S_{t} \Phi\left(d_{1}(t)\right)-K e^{-r(T-t)} \Phi\left(d_{2}(t)\right), \\
& P\left(t, S_{t}\right)=K e^{-r(T-t)} \Phi\left(-d_{2}(t)\right)-S_{t} \Phi\left(-d_{1}(t)\right),
\end{aligned}
$$

\footnotetext{
${ }^{3}$ En general un proceso X es cuadrado integrable bajo $P^{*}$ si $E^{*}\left[\int_{0}^{T} X_{t}^{2} d t\right]<\infty$.
} 
donde

$$
\begin{aligned}
& \Phi(x)=\frac{1}{\sqrt{2 \pi}} \int_{-\infty}^{x} e^{-\frac{y^{2}}{2}} d y \\
& d_{1}(t)=\frac{\ln \left(S_{t} / K\right)+\left(r+\frac{\sigma^{2}}{2}\right)(T-t)}{\sigma \sqrt{T-t}}, \\
& d_{2}(t)=\frac{\ln \left(S_{t} / K\right)+\left(r-\frac{\sigma^{2}}{2}\right)(T-t)}{\sigma \sqrt{T-t}},
\end{aligned}
$$

Demostración. Ver Lamberton y Lampeyre (2008) pág. 69-70.

Note que existe una relación directa entre el valor de un call y un put, conocida como paridad put-call y esta dada por:

$$
C_{t}-P_{t}=K e^{-r(T-t)}-S_{t}
$$

donde el miembro derecho de la ecuación anterior es contrato forward.

\subsection{Ecuación diferencial parcial}

Considérese la ecuación diferencial estocástica (ede) n-dimensional,

$$
d X_{t}=\mu\left(t, X_{t}\right) d t+\sigma\left(t, X_{t}\right) d W_{t}
$$

Por la fórmula de Itô, el proceso anterior esta muy relacionado con un operador diferencial parcial $\mathscr{A}$ llamado operador infinitesimal.

Definición 6. Dada la ede (1.3), el operador diferencial parcial $\mathscr{A}$, llamado operador infinitesimal de $X$ se define para cualquier función $h$, con $h \in C^{1,2}\left([0, T] \times \Re^{n}\right)$ y $(t, x) \in[0, T] \times \Re^{n}$ como:

$$
(\mathscr{A} h)(t, x)=\sum_{i=1}^{n} \mu_{i}(t, x) \frac{\partial h}{\partial x_{i}}(x)+\frac{1}{2} \sum_{i, j=1}^{n} C_{i, j}(t, x) \frac{\partial^{2} h}{\partial x_{i} \partial x j}(x)
$$

donde $C(t, x)=\sigma(t, x) \sigma^{T}(t, x)$ y $t \in[0, T]$. 
Este operador es también conocido como el operador de Dynkin o el operador de Itô, o bien el operador hacia atrás de Kolmogorov. En términos del operador infinitesimal, la fórmula de Itô tiene la siguiente forma:

$$
d h\left(t, X_{t}\right)=\left(\frac{\partial h}{\partial t}+\mathscr{A} h\right) d t+\nabla_{x} h \sigma d W_{t}
$$

donde el gradiente $\nabla_{x}$ es definido para $h \in C^{1}\left(\Re^{n}\right)$ como

$$
\nabla_{x} h=\left[\frac{\partial h}{\partial x_{1}}, \cdots, \frac{\partial h}{\partial x_{n}}\right] .
$$

Existe una relación entre la ecuación diferencial estocástica y la ecuación diferencial parcial parabólica. Por ejemplo si se considera el problema de Cauchy con $n=1$, se tiene que dadas $\mu(t, x), \sigma(t, x)$ y $\Phi(x)$ funciones escalares, el objetivo es encontrar una función $F$ la cual satisfaga el siguiente problema con valor final sobre $[0, T] \times \Re$ :

$$
\begin{aligned}
\frac{\partial F}{\partial t}(t, x)+\mu(t, x) \frac{\partial F}{\partial x}+\frac{1}{2} \sigma^{2}(t, x) \frac{\partial^{2} F}{\partial x^{2}}(t, x) & =0 \\
F(T, x) & =\Psi(x) .
\end{aligned}
$$

Ahora fijando un punto $t$ en el tiempo y un punto $x$ en el espacio, se define el proceso $X$ sobre el intervalo de tiempo $[t, T]$ como la solución de la ede

$$
\begin{aligned}
d X_{t} & =\mu\left(t, X_{t}\right) d t+\sigma\left(t, X_{t}\right) d W_{t} \\
X_{t} & =x
\end{aligned}
$$

Notemos que el operador infinitesimal $\mathscr{A}$ de este proceso está dado por,

$$
\mathscr{A}=\mu(t, x) \frac{\partial}{\partial x}+\frac{1}{2} \sigma^{2}(t, x) \frac{\partial^{2}}{\partial x^{2}}
$$


Por lo tanto la ecuación (1.5) se transforma en el siguiente problema con valor final:

$$
\begin{aligned}
\frac{\partial F}{\partial t}(t, x)+\mathscr{A} F & =0, \\
F(T, x) & =\Psi(x) .
\end{aligned}
$$

Entonces aplicando Itô a $F\left(s, X_{s}\right)$ en el intervalo $[t, T]$ y por la ecuación (1.4) tenemos:

$$
d F(t, x)=\left(\frac{\partial F}{\partial t}\left(t, X_{t}\right)+\mathscr{A} F\left(t, X_{t}\right)\right) d t+\sigma\left(t, X_{t}\right) \frac{\partial F}{\partial x}\left(t, X_{t}\right) d W_{t}
$$

la cual tiene la siguiente forma integral

$$
\begin{aligned}
F\left(T, X_{T}\right)= & F\left(t, X_{t}\right)+\int_{t}^{T}\left(\frac{\partial F}{\partial t}\left(u, X_{u}\right)+\mathscr{A} F\left(u, X_{u}\right)\right) d u+ \\
& +\int_{t}^{T} \sigma\left(u, X_{u}\right) \frac{\partial F}{\partial x}\left(u, X_{u}\right) d W_{u} .
\end{aligned}
$$

De la ecuación anterior podemos notar que dado que $F$ satisface la ecuación (1.7), la integral con respecto al tiempo desaparece y además el término del browniano es integrable, entonces tomando esperanza de ambos lados tenemos:

$$
F\left(t, X_{t}\right)=E_{t, x}\left[\Psi\left(X_{T}\right)\right]
$$

donde $E_{t, x}[\cdot]$ significa que el valor esperado es tomado dado el valor inicial $X_{t}=x$. Entonces se tiene el siguiente resultado conocido como la fórmula de representación estocástica de Feynman-Kac

Proposición 1.2.1. (Feynman-Kac). Supóngase que F es una solución al problema con valor final,

$$
\begin{aligned}
\frac{\partial F}{\partial t}(t, x)+\mu(t, x) \frac{\partial F}{\partial x}+\frac{1}{2} \sigma^{2}(t, x) \frac{\partial^{2} F}{\partial x^{2}}(t, x) & =0 \\
F(T, x) & =\Psi(x) .
\end{aligned}
$$


Además supóngase que el proceso

$$
\sigma\left(u, X_{u}\right) \frac{\partial F}{\partial x}\left(u, X_{u}\right)
$$

esta en $L^{2}$, entonces $F$ tiene la representación

$$
F(t, x)=E_{t, x}\left[\Psi\left(X_{T}\right)\right],
$$

donde X satisface la ede

$$
\begin{aligned}
d X_{u} & =\mu\left(u, X_{u}\right) d u+\sigma(u, X u) d W_{u}, \quad t \leq u \leq T \\
X_{t} & =x
\end{aligned}
$$

A este problema también se le conoce como problema parabólico. Existe un problema con valor final muy relacionado al problema parábolico anterior, por lo tanto se tiene la siguiente proposición:

Proposición 1.2.2. (Feynman-Kac). Supóngase que F es una solución al problema con valor final,

$$
\begin{aligned}
\frac{\partial F}{\partial t}(t, x)+\mu(t, x) \frac{\partial F}{\partial x}+\frac{1}{2} \sigma^{2}(t, x) \frac{\partial^{2} F}{\partial x^{2}}(t, x)-x F & =0 \\
F(T, x) & =\Psi(x) .
\end{aligned}
$$

Además supóngase que el proceso

$$
e^{-\int_{t}^{u} X_{u} d u} \sigma\left(u, X_{u}\right) \frac{\partial F}{\partial x}\left(u, X_{u}\right)
$$

esta en $L^{2}$, entonces $F$ tiene la representación

$$
F(t, x)=E_{t, x}\left[e^{-\int_{t}^{T} X_{u} d u} \Psi\left(X_{T}\right)\right]
$$


donde X satisface la ede

$$
\begin{aligned}
d X_{u} & =\mu\left(u, X_{u}\right) d u+\sigma\left(u, X_{u}\right) d W_{u}, \\
X_{t} & =x .
\end{aligned}
$$

Demostración. Considérese el proceso estocástico $Y(s)=e^{-\int_{t}^{s} X_{u} d u} F\left(s, X_{s}\right)$, entonces aplicando Itô a $Y(s)$ se tiene,

$$
d Y=e^{-\int_{t}^{s} X_{u} d u}\left[\frac{\partial F}{\partial t}+\mu \frac{\partial F}{\partial x}+\frac{1}{2} \sigma^{2} \frac{\partial^{2} F}{\partial x^{2}}-x F\right]+e^{-\int_{t}^{s} X_{u} d u} \sigma \frac{\partial F}{\partial x} d W,
$$

dado que $F$ satisface la ecuación (1.12) entonces,

$$
d Y=e^{-\int_{t}^{s} X_{u} d u} \sigma \frac{\partial F}{\partial x} d W
$$

integrando de expresión anterior de $t$ a $T$

$$
Y_{T}=Y_{t}+\int_{t}^{T} e^{-\int_{t}^{s} X_{u} d u} \sigma \frac{\partial F}{\partial x} d W_{s}
$$

y sustituyendo el valor de $Y(\cdot)$

$$
e^{-\int_{t}^{T} X_{u} d u} F\left(T, X_{T}\right)=F\left(t, X_{t}\right)+\int_{t}^{T} e^{-\int_{t}^{s} X_{u} d u} \sigma \frac{\partial F}{\partial x} d W_{s}
$$

por último tomando esperanza de ambos lado y considerando que los valores finales son $F\left(T, X_{T}\right)=\Psi\left(X_{T}\right)$ y $X_{t}=x$, tenemos

$$
F(t, x)=E_{t, x}\left[e^{-\int_{t}^{T} X_{u} d u} \Psi\left(X_{T}\right)\right]
$$

El siguiente problema aparece con frecuencia en el estudio de las tasas de interés, el cual hace referencia al teorema de Girsanov ${ }^{4}$. Denotemos $\lambda(t, x)$ como la prima por

\footnotetext{
${ }^{4}$ Ver Apéndice A, Teorema A.1.2.
} 
riesgo $^{5}$ y supóngase que $F$ es la solución al problema con valor a la frontera,

$$
\begin{aligned}
\frac{\partial F}{\partial t}(t, x)+[\mu(t, x)-\lambda(t, x) \sigma(t, x)] \frac{\partial F}{\partial x}(t, x)+\frac{1}{2} \sigma^{2}(t, x) \frac{\partial^{2} F}{\partial x^{2}}(t, x)-x F(t, x) & =0 \\
F(T, x) & =\Psi(x),
\end{aligned}
$$

$\mathrm{y}\left(X_{s}\right)_{s \geq 0}$ satisface la ede

$$
\begin{aligned}
d X_{s} & =\mu\left(s, X_{s}\right) d s+\sigma\left(s, X_{s}\right) d W_{s} \\
X_{t} & =x
\end{aligned}
$$

Utilizando la Proposición 1.2.2 para resolver la ecuación diferencial anterior, entonces el proceso $X_{s}$ debe satisfacer,

$$
\begin{aligned}
d X_{s} & =\left[\mu\left(s, X_{s}\right)-\lambda\left(s, X_{s}\right) \sigma\left(s, X_{s}\right)\right] d s+\sigma\left(s, X_{s}\right) d W_{s} \\
X_{t} & =x,
\end{aligned}
$$

lo cual ocurre si

$$
\begin{aligned}
d X_{s} & =\mu d s+\sigma d W_{s} \\
& =(\mu-\lambda \sigma) d s+\lambda \sigma d s+\sigma d W_{s} \\
& =(\mu-\lambda \sigma) d s+\sigma\left(\lambda d s+d W_{s}\right) \\
& =(\mu-\lambda \sigma) d s+\sigma\left(d \tilde{W}_{s}\right),
\end{aligned}
$$

donde $d \widetilde{W}_{s}=\lambda d s+d W_{s}$, entonces utilizando el teorema de Girsanov y la Proposición

\subsection{2 tenemos}

$$
F(t, x)=E_{t, x}\left[\exp \left(-\int_{t}^{T} X_{s} d s-\frac{1}{2} \int_{t}^{T} \lambda^{2}\left(s, X_{s}\right) d s-\int_{t}^{T} \lambda\left(s, X_{s}\right) d W_{s}\right) \Psi\left(X_{T}\right)\right] .
$$

\footnotetext{
${ }^{5}$ La prima de riesgo es la cantidad mínima de dinero que hace que el rendimiento esperado de un activo con riesgo exceda el rendimiento conocido de un activo libre de riesgo.
} 


\subsection{Opciones con barrera}

Las opciones en las que su posibilidad de ejercicio dependerá de que el activo subyacente alcance un determinado nivel (barrera) durante un cierto período de tiempo se conocen con el nombre de opciones con barrera. Si esto ocurre, la opción condicional se convierte en una opción de compra o de venta simple (opciones tipo knock-in), o por el contrario, puede ser que deje de existir desde el momento en que se alcance el nivel barrera (opciones tipo knockout).

- Opciones tipo knock-in. Estas opciones tienen derecho a ejercerse sólo si el valor del subyacente alcanza un determinado nivel. Se clasifican en dos tipos:

- Opciones up and in: se activa el derecho a ejercer la opción al vencimiento cuando el valor del subyacente se sitúa por encima de un determinado nivel durante la vida de la opción.

- Opciones down and in: activa el derecho de ejercicio al vencimiento, si el valor del subyacente cae por debajo de un determinado valor que constituye la barrera.

- Opciones tipo knock-out. Estas opciones tienen derecho a ejercerse sólo si el valor del subyacente no alcanza la barrera, es decir, que desaparece el derecho de ser ejercidas si el valor del subyacente toca la barrera en algún momento de su vida. Se clasifican en dos tipos:

- Opciones up and out: dejan de existir si el valor del subyacente se sitúa por encima de un determinado nivel durante la vida de la opción.

- Opciones down and out: desaparece el derecho de que la opción sea ejercida, si el valor del subyacente se sitúa por debajo del nivel establecido como barrera, el cual.

Considérese el modelo de Black \& Scholes estándar,

$$
\begin{aligned}
d S(t) & =\alpha S(t) d t+\sigma S d W(t), \\
d B(t) & =r B(t) d t,
\end{aligned}
$$


con $\alpha, \sigma>0$ y $r>0$ parámetros fijos. Si el precio de ejercicio es $T$, supongamos se tiene un reclamo contingente $\mathcal{Z}$ de la forma:

$$
\mathcal{Z}=\Theta(S(T))
$$

Denotemos a $F(t, s ; T, \Theta)$ como la función de precios de $\mathcal{Z}$. Fijando un número $L<S(0)$, el cual será nuestra barrera, consideremos $\mathcal{Z}_{L O}$ el correspondiente contrato down and out descrito anteriormente.

Definición 7. Dado un contrato $\mathfrak{Z}_{L O}$ con maduración $T$, entonces $\mathfrak{Z}_{L O}$ está definido por:

$$
\mathscr{Z}_{L O}=\left\{\begin{array}{cc}
\Theta(S(T)) & \text { si } S(t)>L \text { para todo } t \in[0, T] \\
0 & \text { si } S(t) \leq L \text { para algún } t \in[0, T]
\end{array}\right.
$$

Definición 8. Dada una función fija $\Theta$, definamos $\Theta_{L}$ como:

$$
\Theta_{L}(x)=\left\{\begin{array}{cc}
\Theta(x) & \text { para } x>L, \\
0 & \text { para } x \leq L
\end{array},\right.
$$

es decir, $\Theta_{L}(x)=\Theta(x) 1_{\{x>L\}}$. Note que el funcional $F(t, S ; T, \Theta)$ es lineal en el argumento $\Theta$, así como la función $\Theta_{L}$ lo es respecto a la variable $L$ el siguiente lema:

Lema 1.3.1. Para todo real $\alpha$ y $\beta$, y para toda función $\Theta$ y $\Xi$ se tiene que:

$$
\begin{aligned}
F(t, s ; T, \alpha \Theta+\beta \Xi) & =\alpha F(t, s ; T, \Theta)+\beta F(t, s ; T, \Xi), \\
(\alpha \Theta+\beta \Xi)_{L} & =\alpha \Theta_{L}+\beta \Xi_{L} .
\end{aligned}
$$

Demostración. Ver Björk (2004), pág.257.

El principal resultado se muestra en el siguiente teorema, en el cual se muestra que el problema de valuar la versión down-and-out de un contrato $\Theta$ se reduce a la valuación de un contrato sin barrera sobre un reclamo $\Theta_{L}$.

Teorema 1.3.1. (Valuación de contratos down-and-out). Consideré un $\mathcal{Z}=\Theta(S(T)$ ) fijo. Entonces la función de precios, denotada por $F_{L O}$, correspondiente a un contrato 
down-and-out $\mathcal{Z}_{L O}$, para $s>$ L está dada por:

$$
F_{L O}(t, s ; T, \Theta)=F\left(t, s ; T, \Theta_{L}\right)-\left(\frac{L}{s}\right)^{\frac{2\left(r-\frac{1}{2} \sigma^{2}\right)}{\sigma^{2}}} F\left(t, \frac{L^{2}}{s} ; T, \Theta_{L}\right)
$$

Demostración. Ver Björk (2004), pág. 257-259.

También se tiene un resultado sobre la linealidad en la valuación de un contrato down-and-out:

Lema 1.3.2. Para cualquier funciones de contrato $\Theta$ y $\Xi$, y para cualquier número real $\alpha$ y $\beta$ se cumple que:

$$
F_{L O}(t, s ; T, \alpha \Theta+\beta \Xi)=\alpha F_{L O}(t, s ; T, \Theta)+\beta F_{L O}(t, s ; T, \Xi) .
$$

Demostración. Aplicando el Teorema 1.3.1 y el lema 1.3.1 a los operadores $\Theta$ y $\Xi$.

Definición 9. Sea $T$ un plazo $K$ y $L$ parámetros fijos, defínase las siguientes reclamaciones:

$$
\begin{aligned}
S T(x) & =x, \text { para todo } x, \\
B O(x) & =1, \text { para todo } x, \\
H(x ; L) & =\left\{\begin{array}{ll}
1 & \text { si } x>L, \\
0 & \text { si } x \leq L
\end{array},\right. \\
C(x ; K) & =\max [x-K, 0] .
\end{aligned}
$$

El contrato $S T$ ofrece al propietario una unidad del activo subyacente al tiempo $T$, mientras que $B O$ es un bono cupón cero que paga una unidad a la maduración $T$. El contrato $H$ da al propietario una unidad si el valor del activo subyacente excede $L$ al tiempo $T$, y cero si no ocurre. Y por último $C$ es un call europeo con precio de ejercicio $K$.

Tomando las definiciones anteriores, las funciones de pago de los contratos anteriores se muestran en el siguiente lema: 
Lema 1.3.3. La función de precios de los contratos descritos en la Definición 9, están dados por:

$$
\begin{aligned}
\boldsymbol{S T}(t, s) & =s \\
\boldsymbol{B O}(t, x s & =e^{-r(T-t)} \\
\boldsymbol{H}(t, s ; L) & =e^{-r(T-t)} \Phi\left(\frac{\left(r-\frac{1}{2} \sigma^{2}\right)(T-t)+\log (s / L)}{\sigma \sqrt{T-t}}\right) \\
\boldsymbol{C}(x ; K) & =C(t, s),
\end{aligned}
$$

donde $c(t, s)$ es la fórmula de Black \& Scholes vista en el Corolario 1.1.1.

Demostración. El valor de $S T$ al tiempo $t$ es igual al valor del activo subyacente al mismos tiempo, por lo tanto $\mathbf{S T}(t, s)=s$. Mientras que el valor de un bono cupón cero que paga uno al tiempo $t$ es $e^{-r(T-t)}$. Dado que $C$ es la función de pago de un call europeo, entonces la función de precios está dada por la fórmula de Black \& Scholes. Para el caso de $H$ notemos que:

$$
\begin{aligned}
\mathbf{H}(t, s ; L) & =E^{*}\left[e^{r(T-t)} 1_{\{x>L\}}\right] \\
& =e^{r(T-t)} P^{*}(x>L) \\
& =e^{r(T-t)} P^{*}\left(s e^{Y}>L\right) \\
& =e^{r(T-t)} P^{*}(Y>\log (L)-\log (s)) \\
& =e^{r(T-t)} P^{*}\left(\frac{Y-\left(r-\frac{1}{2} \sigma^{2}\right)(T-t)}{\sigma \sqrt{(T-t)}}>\frac{\log (L)-\log (s)-\left(r-\frac{1}{2} \sigma^{2}\right)(T-t)}{\sigma \sqrt{(T-t)}}\right) \\
& =e^{r(T-t)} \Phi\left(\frac{\left(r-\frac{1}{2} \sigma^{2}\right)(T-t)+\log (s / L)}{\sigma \sqrt{T-t}}\right)
\end{aligned}
$$

donde $Y \sim N\left(\left(r-\frac{1}{2} \sigma^{2}\right)(T-t), \sigma^{2}(T-t)\right)$.

A continuación se muestran dos resultados importantes en nuestro estudio, dichos resultados se aplicarán en el modelo de Black-Cox. ${ }^{6}$

\footnotetext{
${ }^{6}$ Ver Cápitulo 2.
} 
Lema 1.3.4. El contrato down-and-out sobre un activo libre de riesgo está dado por:

$$
\begin{aligned}
\boldsymbol{S} \boldsymbol{T}_{L O}(t, s) & =L \cdot \boldsymbol{H}(t, s ; L)-L \cdot\left(\frac{L}{s}\right)^{2 \tilde{r} / \sigma^{2}} \boldsymbol{H}\left(t, \frac{L^{2}}{s} ; L\right) \\
& +\boldsymbol{C}(t, s ; L)-\left(\frac{L}{s}\right)^{2 \tilde{r} / \sigma^{2}} \boldsymbol{C}\left(t, \frac{L^{2}}{s} ; L\right)
\end{aligned}
$$

donde H y están dadas por el Lema (1.3.3).

Demostración. Sea ST $(t, s)=s$ una función de precios y $\tilde{r}=\left(r-\frac{1}{2} \sigma^{2}\right)$, entonces por el Teorema (1.3.1) tenemos

$$
F_{L O}(t, s ; S T)=F\left(t, s ; S T_{L}\right)-\left(\frac{L}{s}\right)^{2 \tilde{r} / \sigma^{2}} F\left(t, \frac{L^{2}}{s} ; S T_{L}\right)
$$

De la Definición 9 podemos encontrar la siguiente relación:

$$
S T_{L}(x)=L \cdot H(x ; L)+C(x ; L) .
$$

Entonces sustituyendo la ecuación anterior en (1.16) tenemos que:

$$
\begin{aligned}
\mathbf{S T}_{L O}(t, s) & =F_{L O}(t, s ; L) \\
& =F(t, s ; L H(; L)+C(; L))-\left(\frac{L}{s}\right)^{2 \tilde{r} / \sigma^{2}} F\left(t, \frac{L^{2}}{s} ; L H(; L)+C(; L)\right) \\
& =L F(t, s ; H(; L))-L \cdot\left(\frac{L}{s}\right)^{2 \tilde{r} / \sigma^{2}} F\left(t, \frac{L^{2}}{s} ; H(; L)\right) \\
& +F(t, s ; C(; L))-\left(\frac{L}{s}\right)^{2 \tilde{r} / \sigma^{2}} F\left(t, \frac{L^{2}}{s} ; C(; L)\right) \\
& =L \cdot \mathbf{H}(t, s ; L)-L \cdot\left(\frac{L}{s}\right)^{2\left(r-\frac{1}{2} \sigma^{2}\right)} \mathbf{H}\left(t, \frac{L^{2}}{s} ; L\right) \\
& +\mathbf{C}(t, s ; L)-\left(\frac{L}{s}\right)^{2\left(r-\frac{1}{2} \sigma^{2}\right)} \mathbf{C}\left(t, \frac{L^{2}}{s} ; L\right)
\end{aligned}
$$

Ahora veamos el resultado para el caso de un contrato down-and-out del tipo call europeo. 
Proposición 1.3.1. El precio de una opción call Europea down-and-out está dado por:

$$
C_{L O}(t, s ; K)=C(t, s ; K)-\left(\frac{L}{s}\right)^{2\left(r-\frac{1}{2} \sigma^{2}\right)} \boldsymbol{C}(t, s ; K), \text { para } L<K,
$$

Demostración. Si $L<K$ entonces de la Definición 9 se tiene que $C_{L}(x, K)=C(x, K)$, por lo tanto aplicando el Teorema 1.3.1 tenemos que:

$$
\begin{aligned}
\mathbf{C}_{L O}(t, s ; L) & =F(t, s ; C(; K))-\left(\frac{L}{s}\right)^{2\left(r-\frac{1}{2} \sigma^{2}\right)} F(t, s ; C(; K)) \\
& =\mathbf{C}(t, s ; K)-\left(\frac{L}{s}\right)^{2\left(r-\frac{1}{2} \sigma^{2}\right)} \mathbf{C}(t, s ; K) .
\end{aligned}
$$

\subsection{Modelos de tasa de interés}

Sea $B(t, T)$ el precio al tiempo $t$ de un bono descontado, es decir, un bono cupón cero que paga una unidad monetaria al tiempo $T$, entonces en particular $B(T, T)=1$. Al tiempo $t$, el rendimiento al vencimiento $R(t, T)$ del bono descontado $B(t, T)$ es la tasa de rendimiento compuesto continuamente que hace que el precio del bono sea uno al tiempo $T$,

$$
B(t, T) e^{(T-t) R(t, T)}=1
$$

o bien

$$
R(t, T)=-\frac{\ln (B(t, T))}{T-t}
$$

Cuando se fija $t$ y $T$ aumenta, la forma de $R(t, T)$ determina la estructura temporal de las tasas de interés. En este caso, la curva de rendimientos es lo mismo que la estructura temporal.

La mayoría de los modelos basados en arbitraje derivan o asumen que un sólo factor es suficiente para explicar la estructura temporal y así suponen que la tasa spot instantánea es la única variable de estado. Existe otros modelos los cuales se supone la 
existencia de otro factor, por lo general la segunda variable es la tasa larga o la tasa de inflación.

Denotemos por $V(t)$ el valor al tiempo $t$ del reclamo contingente que depende de la tasa corta con maduración $t$. Supóngase que bajo la medida de probabilidad $P$, la dinámica de la tasa corta está dada por:

$$
d r(t)=\mu(t, r(t)) d t+\sigma(t, r(t)) d W
$$

Ahora denotemos $B(t, T) \equiv B(t, r(t), T)$, por lo tanto el precio $B(t, r(t), T)$ del bono está junto con todos sus derivados, completamente determinado por la ecuación ${ }^{7}$ general:

$$
B_{1}+(\mu-\lambda \sigma) B_{2}+\frac{1}{2} \sigma^{2} B_{22}-r B=0
$$

Aparte de $\sigma$, el término $\mu-\lambda \sigma$ determina el precio del bono en la ecuación anterior. El término $\mu-\lambda \sigma$ es el término de tendencia (drift) de la tasa corta bajo la medida martingala $P^{*}$.

Definiendo $V_{i}^{*}(t)$ el precio relativo del activo $V$ bajo la numerario $N$ al tiempo $t$, como

$$
V_{i}^{*}(t)=\frac{V(t)}{N(t)}
$$

Proposición 1.4.1. Bajo ciertas condiciones de regularidad, un mercado es libre de arbitraje si existe una medida martingala equivalente $P^{*}$, tal que el proceso de precios relativo de cualquier valor es una $P^{*}$-martingala, es decir,

$$
E^{*}\left[V_{i}^{*}(T) \mid \mathscr{F}_{t}\right]=V^{*}(t)
$$

Demostración. Ver Karatzas y Shreve (1991), pág. 232.

Un ejemplo de numeraria sería el precio al tiempo $t$ de una unidad monetaria reinvertida continuamente a la tasa corta dado un tiempo inicial específico $\tau$, denotada por

\footnotetext{
${ }^{7}$ Ver Cox, Ingersoll y Ross (1985)
} 
$\beta(t)$

$$
\beta(t)=e^{\int_{u}^{t} r(s) d s}
$$

Bajo esta numeraria, el precio relativo del activo $V$ está dado por:

$$
V^{*}(t)=\frac{V(t)}{\beta(t)}
$$

Al usar la proposición anterior, en donde $V$ es un bono cupón cero con maduración $T$ y la numeraria es $\beta(t)$ con $u=t$, tenemos

$$
E^{*}\left[\frac{B(T, T)}{\beta(T)} \mid \mathscr{F}_{t}\right]=\frac{B(t, T)}{\beta(t)}=B(t, T),
$$

y como $B(T, T)=1$ se tiene que

$$
E^{*}\left[e^{-\int_{t}^{T} r(s) d s} \mid \mathscr{F}_{t}\right]=B(t, T)
$$

Analizaremos dos modelos en los cuales $\int_{u}^{t} r(s) d s$ tiene distribución normal. Una variable aleatoria $X$ con distribución nomal $N\left(\mu, \sigma^{2}\right)$ tiene función generadora de momentos

$$
E\left(e^{a X}\right)=e^{a \mu+\frac{1}{2} \sigma^{2} a^{2}} .
$$

Por lo tanto, se tiene una expresión analítica para el precio del bono. En general se tiene que para cualquier derivado $V(t)$ de una tasa de interés que madura al tiempo $T$,

$$
V(t)=E^{*}\left[V(T) e^{-\int_{T}^{t} r(s) d s} \mid \mathscr{F}_{t}\right]
$$

es decir, bajo $P^{*}$ los precios descontados de los activos son martingala.

En la literatura existen diversas propuestas para especificar la $P^{*}$-dinámica de la tasa corta $r$. A continuación se presentan los modelos más utilizados, en donde si un parámetro depende del tiempo se expresa explícitamente; en otro caso se toma como constante. 
1. Merton

$$
d r(t)=\mu d t+\sigma d W(t), \quad \mu>0, \sigma>0
$$

2. Vasicek

$$
d r(t)=k(\theta-r(t)) d t+\sigma d W(t), \quad k>0, \theta>0, \sigma>0 .
$$

3. Cox-Ingersoll-Ross(CIR)

$$
d r(t)=k(\theta-r(t)) d t+\sigma \sqrt{r(t)} d W(t) \quad k>0, \theta>0, \sigma>0 .
$$

Existen más modelo para la tasa de interés corta tales como el modelo de Dothan, Black-Derman-Toy, Ho-Lee y Hull-White, pero en este trabajo nos enfocaremos en los modelos de Merton, Vasicek y CIR para valuar bonos cupón cero.

\subsubsection{Modelo de Merton (1973)}

En este modelo, Robert C. Merton propone que la dinámica de la tasa corta está dada por:

$$
d r(t)=\mu d t+\sigma d W(t)
$$

donde $\mu$ y $\sigma$ son constantes y $W(t)$ es un movimiento browniano estándar. Además supone que el premio al riesgo $\lambda$ es constante. La solución de la ecuación anterior con $t \leq u$ es

$$
r(u)=r(t)+\mu(u-t)+\sigma \int_{t}^{u} d W(z)
$$

Dada la información al tiempo $t, r(t)$ se distribuye normal con media (condicional)

$$
E\left[r(u) \mid \mathscr{F}_{t}\right]=r(t)+\mu(u-t),
$$


y varianza (condicional)

$$
\operatorname{Var}\left[r(u) \mid \mathscr{F}_{t}\right]=\sigma^{2}(u-t)
$$

Si denotamos a $I(t, T)=\int_{t}^{T} r(s) d s$, entonces $I(t, T)$ tiene distribución normal con media

$$
E\left[I(t, T) \mid \mathscr{F}_{t}\right]=r(t)(T-t)+\frac{1}{2} \mu(T-t)^{2},
$$

y varianza

$$
\operatorname{Var}\left[I(t, T) \mid \mathscr{F}_{t}\right]=\sigma^{2} \int_{t}^{T}(T-s)^{2} d s=\frac{1}{3} \sigma^{2}(T-t)^{3}
$$

Para calcular el valor de la varianza se utiliza la propiedad de isometría de Itô,

$$
E\left[\left(\int_{t}^{T} f(s) d W(s)\right)^{2} \mid \mathscr{F}_{s}\right]=\int_{t}^{T} f^{2}(s) d s
$$

Dado que $I(t, T)$ tiene distribución normal, entonces el precio del bono $B(t, r(t), T)$ satisface,

$$
\begin{aligned}
B(t, r(t), T) & =E\left[e^{-I(t, T)} \mid \mathscr{F}_{t}\right] \\
& =e^{-r(t)(T-t)-\frac{1}{2} \mu(T-t)^{2}+\frac{1}{6} \sigma^{2}(T-t)^{3}} .
\end{aligned}
$$

Mientras que la estructura temporal de la tasa de interés está dada por

$$
\begin{aligned}
R(t, T) & =-\frac{\ln (B(t, r(t), T))}{T-t} \\
& =r(t)+\frac{1}{2} \mu(T-t)-\frac{1}{6} \sigma^{2}(T-t)^{2} .
\end{aligned}
$$

Se observa que la tasa de interés con maduración infinita diverge, esto es

$$
\lim _{T \rightarrow \infty} R(t, T)=-\infty
$$

Podemos notar que el primer y segundo momento de la distribución de la tasa $r(t)$ 
no están acotados por lo tanto se permite que la tasa de interés $R(t, T)$ pueda ser negativa o infinita respecto a $T$. Este modelo no presenta reversión a la media, entonces la tasa corta no regresará a un nivel de largo plazo conforme el tiempo transcurre.

\subsubsection{Modelo de Vasicek (1977)}

El modelo de Vasicek propone modelar la tasa de interés de corto plazo con un proceso de Ornstein-Uhlenbeck, dado por:

$$
d r(t)=k(\theta-r(t)) d t+\sigma d W(t)
$$

donde $k, \theta$ y $\sigma$ son constantes positivas y $W(t)$ es un movimiento browniano estándar. En este modelo se define una caminata aleatoria alrededor de una tendencia, y por tanto tiene reversión a la media.

Definiendo $m(t)=k r(t)-k \theta$, el modelo de Vasicek puede reescribirse como

$$
d m(t)=-k m(t) d t+k \sigma d W(t)
$$

La ecuación (1.17) es conocida como el proceso de Ornstein-Uhlenbeck y la solución aplicando Itô al proceso $X(t)=m(t) e^{k t}$ está dada por,

$$
m(t)=m(s) e^{-k(t-s)}+k \sigma \int_{s}^{t} e^{-k(t-u)} d W(u), s<t
$$

Y como $m(t)=k r(t)-k \theta$ se tiene que

$$
r(t)=\theta+(r(s)-\theta) e^{-k(t-s)}+\sigma \int_{s}^{t} e^{-k(t-u)} d W(u), \quad \forall s \leq t .
$$

Dada la información al tiempo $s, r(t)$ se distribuye normal con media (condicional)

$$
E\left[r(t) \mid \mathscr{F}_{s}\right]=\theta+(r(s)-\theta) e^{-k(t-s)},
$$


y varianza (condicional)

$$
\begin{aligned}
\operatorname{Var}\left[r(t) \mid \mathscr{F}_{s}\right] & =\sigma^{2} \int_{s}^{t} e^{-2 k(t-u)} d u, \\
& =\frac{\sigma^{2}}{2 k}\left(1-e^{-2 k(t-s)}\right)
\end{aligned}
$$

En este modelo, al igual que en el modelo de Merton, las tasas de interés podrían ser negativas. Pero en este caso para valores grandes de $t$, el valor esperado y la varianza de la tasa corta son $\theta$ y $\frac{\sigma^{2}}{2 k}$, por lo tanto el proceso de reversión a la media evita que estos dos valores diverjan.

Sea $I(t, T)=\int_{t}^{T} r(s) d s$, entonces $I(t, T)$ tiene una distribución normal con media

$$
E\left[I(t, T) \mid \mathscr{F}_{t}\right]=\theta(T-t)+(r(t)-\theta)\left(\frac{1-e^{-k(T-t)}}{k}\right)
$$

y varianza

$$
\begin{aligned}
\operatorname{Var}\left[I(t, T) \mid \mathscr{F}_{t}\right] & =\sigma^{2} \int_{t}^{T}\left(\frac{1-e^{-k(T-u)}}{k}\right)^{2} d u, \\
& =\frac{\sigma^{2}}{k^{2}}\left(T-t-2\left(\frac{1-e^{-k(T-t)}}{k}\right)+\frac{1}{2 k}\left(1-e^{-2 k(T-t)}\right)\right) .
\end{aligned}
$$

Y el precio del bono $B(t, r(t), T)$ satisface,

$$
\begin{aligned}
B(t, r(t), T) & =E[-I(t, T) \mid \mathscr{F} t] \\
& =e^{a(t, T)-r(t) b(t, T)},
\end{aligned}
$$

donde

$$
b(t, T)=\frac{1-e^{-k(T-t)}}{k},
$$

$\mathrm{y}$

$$
a(t, T)=\frac{1}{k^{2}}(b(t, T)-T+t)\left(k^{2} \theta-\frac{1}{2} \sigma^{2}\right)-\frac{\sigma^{2} b^{2}(t, T)}{4 k}
$$


Así la estructura temporal está dada por

$$
\begin{aligned}
R(t, T) & =-\frac{\ln (B(t, T)}{T-t} \\
& =\frac{r(t) b(t, T)-a(t, T)}{T-t} \\
& =r(t) \frac{1-e^{-k(T-t)}}{k(T-t)}-\left(\frac{1-e^{-k(T-t)}}{k(T-t)}-1\right)\left(\theta-\frac{\sigma^{2}}{2 k^{2}}\right)+\frac{\sigma^{2}\left(1-e^{-k(T-t)}\right)^{2}}{4 k^{3}(T-t)} .
\end{aligned}
$$

Se observa que la tasa de interés con maduración infinita está dada por,

$$
\lim _{T \rightarrow \infty} R(t, T)=\theta-\frac{\sigma^{2}}{2 k^{2}}
$$

\subsubsection{Modelo de Cox, Ingersoll, Ross (1985)}

Tanto el modelo de Vasicek y el modelo de Cox, Ingersoll, Ross describen el comportamiento de la tasa corta con reversión a la media. Suponga que la tasa corta sigue el proceso

$$
d r(t)=k(\theta-r(t)) d t+\sigma(r(t))^{\beta} d W(t)
$$

donde $k, \theta$ y $\sigma$ son constantes positivas y $W(t)$ es un movimiento browniano estándar. Los modelos de Vasicek y CIR son resultado de tomar $\beta=0$ y $\beta=1 / 2$; respectivamente. Por lo tanto el modelo CIR propone que la tasa de interés corta satisfaga

$$
d r(t)=k(\theta-r(t)) d t+\sigma \sqrt{r(t)} d W(t), \beta \geq 0
$$

y el premio al riesgo está dado por $\lambda(t, r)=\lambda \sqrt{r(t)}$.

Este proceso es conocido como proceso de raíz cuadrada, similar al proceso de Vasicek, pero la varianza es proporcional a $\sigma^{2} t$ por unidad de tiempo.

Utilizando Itô para el proceso $X(t)=r(t) e^{k t}$ se obtiene la solución a la ecuación diferencial estocástica (1.20),

$$
r(t)=\theta+(r(s)-\theta) e^{-k(t-s)}+\sigma \int_{s}^{t} e^{-k(t-u)} \sqrt{r(u)} d W(u), \quad \forall t \geq s .
$$


A diferencia de los modelos anteriores, la tasa corta en el modelo CIR se distribuye como un ji-cuadrada $\left(\chi^{2}\right)$, entonces la tasa $r(t)$ siempre es positiva.

Se puede demostrar que la esperanza y la varianza de $r(t)$ están dadas por:

$$
E\left[r(t) \mid \mathscr{F}_{s}\right]=\theta+(r(s)-\theta) e^{-k(t-s)},
$$

$\mathrm{y}$

$$
\operatorname{Var}\left[r(t) \mid \mathscr{F}_{s}\right]=\frac{\sigma^{2} \theta}{2 k}\left(1-e^{-k(t-s)}\right)^{2}+\frac{\sigma^{2} r(s)}{k}\left(e^{-k(t-s)}-e^{-2 k(t-s)}\right)
$$

Y por lo tanto cuando $t \rightarrow \infty$ la esperanza y varianza tiene el siguiente comportamiento:

$$
\lim _{t \rightarrow \infty} E\left[r(t) \mid \mathscr{F}_{s}\right]=\theta
$$

$\mathrm{y}$

$$
\lim _{t \rightarrow \infty} \operatorname{Var}\left[r(t) \mid \mathscr{F}_{s}\right]=\theta \frac{\sigma^{2}}{2 k}
$$

El precio del bono en este modelo esta dado por,

$$
\begin{aligned}
B(t, r(t), T) & =E^{*}\left[-\int_{t}^{T} r(s) d s \mid \mathscr{F}_{t}\right] \\
& =e^{a(t, T)-r(t) b(t, T)}
\end{aligned}
$$

donde

$$
b(t, T)=\frac{2\left(e^{\gamma T}-1\right)}{(\gamma+k)\left(e^{\gamma T}-1\right)+2 \gamma},
$$

$\mathrm{y}$

$$
a(t, T)=\ln \left(\frac{2 \gamma e^{(k+\gamma)(T / 2)}}{(\gamma+k)\left(e^{\gamma T}-1\right)+2 \gamma}\right)^{2 k \theta / \sigma^{2}}
$$

$\operatorname{con} \gamma=\sqrt{k^{2}+2 \sigma^{2}}$. 
Entonces la estructura temporal está dada por

$$
\begin{aligned}
R(t, T) & =-\frac{\ln (B(t, T)}{T-t} \\
& =\frac{b(t, T) r(t)}{T-t}-\frac{\ln (a(t, T))}{T-t}
\end{aligned}
$$

y se puede mostrar que la tasa de interés con maduración infinita está dada por,

$$
\lim _{T \rightarrow \infty} R(t, T)=\frac{2 k \theta}{k+\lambda \sigma+\gamma}
$$

\subsection{Estimación de parámetros para modelos de tasa de interés}

El proceso de estimación de parámetros en un modelo estocástico permite conocer características adicionales que la estructura por sí misma no es capaz de capturar. La importancia implícita que posee la estimación de parámetros recae en el hecho de que en la mayoría de las aplicaciones reales los parámetros no pueden ser establecidos a priori, teniendo que ser inferidos a partir del comportamiento histórico de las series temporales analizadas.

Existen técnicas para la estimación de los parámetros en los modelos de tasa de interés, entre las principales se encuentran: el Método de Momentos, Mínimos Cuadrado Ordinarios y Máxima Verosimilitud. En este trabajo se presentan los estimadores por mínimos cuadrados y por máxima verosimilitud para los modelos de Vasicek y CIR.

\subsubsection{Modelo Vasicek}

Consideremos el modelo de Vasicek en donde la tasa corta $r$ tiene la siguiente dinámica,

$$
d r(t)=k(\theta-r(t)) d t+\sigma d W(t)
$$

Para la estimación por mínimos cuadrados, se toma la discretización de primeras diferencias por el método de Euler en un período de tiempo $T$ y utilizando las propiedades 
del movimiento browniano tenemos,

$$
r_{t}-r_{t-1} \approx k\left(\theta-r_{t-1}\right) \Delta t+\sigma \sqrt{\Delta t} \epsilon_{t}
$$

donde $\epsilon_{t} \sim N(0,1)$ y $\Delta t=T / n$. Reescribiendo (1.21)

$$
r_{t}=k \theta \Delta t+(1-k \Delta t) r_{t-1}+\sigma \sqrt{\Delta t} \epsilon_{t}
$$

O de manera equivalente,

$$
r_{t}=\alpha+\beta r_{t-1}+\eta_{t}
$$

con $\eta_{t} \sim N\left(0, \sigma^{2} \Delta t\right)$. Por lo tanto utilizando mínimos cuadrados, obtenemos los estimadores de $\hat{\alpha}$ y $\hat{\beta}$. Con estos valores es posible determinar la estimación de $k$ y $\theta$, de manera que la estimación de $\sigma$ es la desviación estándar de los residuales de (1.22).

La estimación por el método de mínimos cuadrados es inadecuado en términos generales, e incluso cuando la regresión puede ser un método teóricamente válido, sus fallos en las aplicaciones prácticas están bien documentada ${ }^{8}$.

Dado que $r_{t}$ sigue un proceso gaussiano donde la media y varianza están dadas por (1.18) y (1.19) respectivamente, se tiene que la función de densidad de probabilidad $p$ de $r_{t}$ con tasa inicial $r_{t}=x$ está dada por:

$$
p(t, y ; \tau, x)=\sqrt{\frac{k}{\pi \sigma^{2}\left(1-e^{-2 k(t-\tau)}\right)}} \exp \left(\frac{-k\left[(y-\theta)-(x-\theta) e^{-k(t-\tau)}\right]^{2}}{\sigma^{2}\left(1-e^{-2 k(t-\tau)}\right)}\right) .
$$

Tomando como base Xie (2008) para determinar los valores de los parámetros del modelo usando el método de máxima verosimilitud, sean

$$
\begin{aligned}
\alpha & =\frac{k}{\sigma^{2}\left(1-e^{-2 k t}\right)} \\
\beta & =\left(1-e^{-2 k t}\right),
\end{aligned}
$$

\footnotetext{
${ }^{8}$ Ball y Torous (1996).
} 
y definamos

$$
P:=\ln p=-\ln \sqrt{\pi}+\frac{1}{2} \ln \alpha-\alpha[x-y+\beta(\theta-x)]^{2} .
$$

Entonces diferenciando $P$ respecto a $\alpha, \beta$ y $\theta$ tenemos

$$
\begin{aligned}
& \frac{\partial P}{\partial \alpha}=\frac{1}{2 \alpha}-[(x-y)+\beta(\theta-x)]^{2} \\
& \frac{\partial P}{\partial \beta}=-2 \alpha[(x-y)+\beta(\theta-x)](\theta-x) \\
& \frac{\partial P}{\partial \theta}=-2 \alpha[(x-y)+\beta(\theta-x)] \beta .
\end{aligned}
$$

Sea $n$ es tamaño de la muestra, es decir, los datos de mercado de la tasa corta $r$, entonces si definimos $X=\left(r_{0}, \ldots, r_{n-1}\right)$ y $Y=\left(r_{1} \ldots, r_{n}\right)$ por la definición de máxima verosimilitud tenemos que encontrar $\alpha, \beta$ y $\theta$ tal que

$$
\begin{aligned}
\frac{n}{2 \alpha}-\sum_{i=1}^{n}\left[\left(X_{i}-Y_{i}\right)+\beta\left(\theta-X_{i}\right)\right]^{2} & =0 \\
\sum_{i=1}^{n}\left[\left(X_{i}-Y_{i}\right)+\beta\left(\theta-X_{i}\right)\right]\left(\theta-X_{i}\right) & =0 \\
\sum_{i=1}^{n}\left[\left(X_{i}-Y_{i}\right)+\beta\left(\theta-X_{i}\right)\right] & =0 .
\end{aligned}
$$

La solución del sistema anterior esta dado por:

$$
\begin{aligned}
\frac{n}{2 \alpha} & =\left(\sum_{i=1}^{n} X_{i}\right)^{2} \sum_{i=1}^{n}\left(Y_{i}^{2}\right)+\sum_{i=1}^{n}\left(X_{i}^{2}\right)\left(\sum_{i=1}^{n} Y_{i}\right)^{2}+2 \sum_{i=1}^{n} X_{i} \sum_{i=1}^{n} Y_{i} \sum_{i=1}^{n} X_{i} Y_{i} \\
& +n\left[\left(\sum_{i=1}^{n} X_{i} Y_{i}\right)^{2}-\left(\sum_{i=1}^{n} X_{i}\right)^{2}\left(\sum_{i=1}^{n} Y_{i}\right)^{2}\right] \\
\beta & =\frac{\sum_{i=1}^{n} X_{i} \sum_{i=1}^{n}\left(X_{i}-Y_{i}\right)-n \sum_{i=1}^{n}\left[X_{i}\left(X_{i}-Y_{i}\right)\right]}{\left(\sum_{i=1}^{n} X_{i}\right)^{2}-n \sum_{i=1}^{n}\left(X_{i}\right)^{2}} \\
\theta & =\frac{\sum_{i=1}^{n} X_{i} \sum_{i=1}^{n}\left(X_{i} Y_{i}\right)-n \sum_{i=1}^{n}\left(X_{i}\right)^{2} \sum_{i=1}^{n} Y_{i}}{\left(\sum_{i=1}^{n} X_{i}\right) \sum_{i=1}^{n}\left(X_{i}-Y_{i}\right)-n \sum_{i=1}^{n}\left[X_{i}\left(X_{i}-Y_{i}\right)\right]}
\end{aligned}
$$

por lo tanto la estimación de los parámetros originales $k, \theta$ y $\sigma^{2}$ son: 


$$
\begin{aligned}
k & =\frac{1}{t} \ln \frac{\left(\sum_{i=1}^{n} X_{i}\right)^{2}-n \sum_{i=1}^{n}\left(X_{i}^{2}\right)}{\left(\sum_{i=1}^{n} X_{i}\right)\left(\sum_{i=1}^{n} Y_{i}\right)-n \sum_{i=1}^{n}\left(X_{i} Y_{i}\right)} \\
\theta & =\frac{\left(\sum_{i=1}^{n} X_{i}\right) \sum_{i=1}^{n}\left(X_{i} Y_{i}\right)-n \sum_{i=1}^{n}\left(X_{i}^{2}\right)\left(\sum_{i=1}^{n} Y_{i}\right)}{\left(\sum_{i=1}^{n} X_{i}\right) \sum_{i=1}^{n}\left(X_{i}-Y_{i}\right)-n \sum_{i=1}^{n}\left[X_{i}\left(X_{i}-Y_{i}\right)\right]} \\
\sigma^{2} & =\frac{2}{n t}\left[\left(\sum_{i=1}^{n} X_{i}\right)^{2}\left(\sum_{i=1}^{n} Y_{i}^{2}\right)+\sum_{i=1}^{n}\left(X_{i}^{2}\right)\left(\sum_{i=1}^{n} Y_{i}\right)^{2}+2 \sum_{i=1}^{n} X_{i} \sum_{i=1}^{n} Y_{i} \sum_{i=1}^{n}\left(X_{i} Y_{i}\right)\right. \\
& \left.+n\left[\sum_{i=1}^{n}\left(X_{i} Y_{i}\right)^{2}-\sum_{i=1}^{n}\left(X_{i}^{2}\right) \sum_{i=1}^{n}\left(Y_{i}^{2}\right)\right]\right] \\
& \times \frac{\left[\left(\sum_{i=1}^{n} X_{i}\right)^{2}-n \sum_{i=1}^{n}\left(X_{i}^{2}\right)\right]^{2}}{\left[\left(\sum_{i=1}^{n} X_{i}\right)^{2}-n \sum_{i=1}^{n}\left(X_{i}^{2}\right)\right]^{2}-\left[\left(\sum_{i=1}^{n} X_{i}\right)\left(\sum_{i=1}^{n} Y_{i}\right)-n \sum_{i=1}^{n}\left(X_{i} Y_{i}\right)\right]^{2}} \\
& \times \ln \frac{\left(\sum_{i=1}^{n} X_{i}\right)^{2}-n \sum_{i=1}^{n}\left(X_{i}^{2}\right)}{\left(\sum_{i=1}^{n} X_{i}\right)\left(\sum_{i=1}^{n} Y_{i}\right)-n \sum_{i=1}^{n}\left(X_{i} Y_{i}\right)} .
\end{aligned}
$$

\subsubsection{Modelo CIR}

En el modelo CIR se asume que la tasa corta $r$ tiene la siguiente dinámica,

$$
d r(t)=k(\theta-r(t)) d t+\sigma(r(t))^{1 / 2} d W(t)
$$

Este modelo se puede estimar econométricamente mediante el ajuste del siguiente modelo de regresión de una serie de tiempo de la tasa corta.

Sea $x \equiv 2 \sqrt{r}$, entonces por el cálculo de Itô:

$$
d x=\left(\left(2 k \theta-\frac{1}{2} \sigma^{2}\right) \frac{1}{x}-\frac{k}{2} x\right) d t+\sigma d W
$$

notemos que en la expresión anterior ha desaparecido $x$ de la parte estocástica de la ecuación, lo que implica que al discretizar no habrá problemas de contemporalidad entre los residuales y los regresores. Por lo tanto tenemos que $d x \approx x_{t}-x_{t-1}$, lo que nos lleva a

$$
x_{t} \approx\left(\left(2 k \theta-\frac{1}{2} \sigma^{2}\right) \frac{1}{x_{t-1}}-\frac{k}{2} x_{t-1}\right) \Delta t+\eta_{t}
$$


$\operatorname{con} \eta_{t} \sim N\left(0, \sigma^{2}\right)$. En este caso se ajusta una regresión por mínimos cuadrados del tipo

$$
x_{t}=\alpha \frac{1}{x_{t-1}}+\beta x_{t-1}+\eta_{t}
$$

Una vez obtenidos los valores de la estimación de $\alpha$ y $\beta$, se obtiene que:

$$
\begin{aligned}
\hat{k} & =2(1-\hat{\beta}), \\
\hat{\theta} & =\frac{1}{2 \hat{k}}\left(\hat{\alpha}+\frac{\hat{\sigma}^{2}}{2}\right),
\end{aligned}
$$

donde $\hat{\sigma}^{2}$ se estima con la varianza de los residuales de la regresión.

El proceso CIR es un caso de proceso de difusión, en donde la función de densidad tiene un forma cerrada. Dado un $r_{t}$ al tiempo $t$, la densidad de $r_{t+\Delta t}$ al tiempo $t+\Delta t$ se puede ver como,

$$
p\left(r_{t+\Delta t} \mid r_{t} ; k, \theta, \sigma, \Delta t\right)=c e^{-u-v}\left(\frac{v}{u}\right)^{\frac{q}{2}} I_{q}(2 \sqrt{u v})
$$

donde

$$
\begin{aligned}
c & =\frac{2 k}{\sigma^{2}\left(1-e^{-k \Delta t}\right)} \\
u & =c r_{t} e^{-k \Delta t} \\
v & =c_{r} t+\Delta t \\
q & =\frac{2 k \theta}{\sigma^{2}}-1
\end{aligned}
$$

e $I_{q}(\cdot)$ es la función modificada de Bessel de primer tipo y orden $q$. Para ver con mayor detalle la derivación de la función de densidad (1.24), ver Feller (1951), pág. 173-182 .

La función de verosimilitud para la tasa de interés de una serie de tiempo de $N$ observaciones esta dada por:

$$
L(k, \theta, \sigma)=\prod_{i=1}^{N-1} p\left(r_{t+1} \mid r_{t} ; k, \theta, \sigma, \Delta t\right),
$$


por lo tanto la función log-verosimilitud del proceso CIR es de la forma,

$$
\ln L(k, \theta, \sigma)=(N-1) \ln c+\sum_{i=1}^{N-1}\left\{-u_{t_{i}}-v_{t_{i+1}}+.5 q \ln \left(\frac{v_{t_{i+1}}}{u_{t_{i}}}\right)+\ln \left[I_{q}\left(2 \sqrt{u_{t_{i}} v_{t_{i+1}}}\right)\right]\right\}
$$

donde $u_{t_{i}}=c r_{t_{i}} e^{-k \Delta t} \mathrm{y} v_{t_{i+1}}=c r_{t_{i+1}}$.

Dado que la función logaritmo es monótona creciente, al máximizar la función logverosimil también se máximiza la función de verosimilitud. La solución del problema anterior tiene la siguiente forma:

$$
(\hat{k}, \hat{\theta}, \hat{\sigma})=\arg \max _{(k, \theta, \sigma)} \ln L(k, \theta, \sigma) .
$$




\section{Capítulo 2}

\section{Valuación de bonos corporativos}

\subsection{Modelo estructural de Merton}

El primer supuesto del modelo de Black-Scholes-Merton es acerca del proceso estocástico para el precio subyacente del contrato. En el artículo original de Black \& Scholes (1973) se asume que el activo con riesgo no paga dividendos, la tasa de interés libre de riesgo es una constante conocida y la dinámica de los precios sigue un Movimiento browniano geométrico (MBG). Este modelo asume una estructura de capital simple.

Asumiendo los supuestos del modelo de Black \& Scholes, consideremos una empresa que posee activos con valor $V$ que se financian a través de la emisión de acciones con valor $S$ y deuda con valor de mercado $D$. La deuda de la empresa se representa con un bono cupón cero con fecha de vencimiento $T$ y valor nominal $F>0$.

Para todo $t \leq T, V_{t}$ esta dada por:

$$
V_{t}=S_{t}+D_{t}
$$

es decir, el valor de la empresa es igual a la suma de sus obligaciones. Asumiendo que $V_{t}$ sigue un movimiento browniano geométrico con $r$ la tasa de interés libre de riesgo, se tiene que

$$
d V_{t}=V_{t}\left(r d t+\sigma_{V} d \widetilde{W}_{t}\right) \quad V_{0}=0
$$


donde $\left(\widetilde{W}_{t}\right), t \in[0, \infty)$ es un movimiento browniano estándar bajo $P^{*}$ y la volatilidad $\sigma_{V}$ se asume constante. La ecuación (2.2) es equivalente a:

$$
V_{t}=V_{0} e^{\left(r-\frac{\sigma^{2}}{2}\right) t+\sigma \widetilde{W}_{t}}
$$

En el modelo de Merton, los acreedores no pueden forzar a la empresa a declararse en quiebra antes del tiempo de madurez $T$. La empresa incumple si, al tiempo $T$ el valor de la empresa es menor que su deuda. En caso de incumplimiento los acreedores toman el control de la empresa. En otras palabras, el pago al tenedor del bono al tiempo de vencimiento es el $\min \left(V_{T}, F\right)$ que es el valor de mercado de la deuda de la empresa al tiempo $T$.

Teorema 2.1.1. En el modelo de Merton, bajo el modelo de Black \& Scholes, los valores del bono riesgoso $D$ y de la acción $S$ al tiempo $t=0$ están dados por:

$$
\begin{aligned}
D_{0} & =F e^{-r T} \Phi\left(d_{2}\right)+V_{0} \Phi\left(-d_{1}\right), \\
S_{0} & =V_{0} \Phi\left(d_{1}\right)-F e^{-r T} \Phi\left(d_{2}\right),
\end{aligned}
$$

con

$$
\begin{aligned}
\Phi(x) & =\frac{1}{\sqrt{2 \pi}} \int_{-\infty}^{x} e^{-\frac{y^{2}}{2}} d y, \\
d_{1} & =\frac{\ln \left(V_{0} / F\right)+\left(r+\frac{\sigma_{V}^{2}}{2}\right)(T)}{\sigma_{V} \sqrt{T}}, \\
d_{2} & =\frac{\ln \left(V_{0} / F\right)+\left(r-\frac{\sigma_{V}^{2}}{2}\right)(T)}{\sigma_{V} \sqrt{T}}
\end{aligned}
$$

Demostración. Por el Teorema 1.1.1, el valor del bono riesgoso $D_{t}$ para $t<T$ está dado por:

$$
D_{t}=E^{*}\left[e^{-r(T-t)} \min \left(V_{T}, F\right) \mid \mathscr{F}_{t}\right]
$$

donde $\mathscr{F}_{t}$ es la filtración natural y la constante $r$ es la tasa de interés instantánea libre 
de riesgo. Además notemos que la función de pago puede ser vista como:

$$
\min \left(V_{T}, F\right)=F-\max \left(F-V_{T}, 0\right)
$$

por lo tanto

$$
\begin{aligned}
D_{t} & =E^{*}\left[e^{-r(T-t)} \min \left(V_{T}, F\right) \mid \mathscr{F}_{t}\right], \\
& =F e^{-r(T-t)}-E^{*}\left[e^{-r(T-t)} \max \left(F-V_{T}, 0\right) \mid \mathscr{F}_{t}\right]
\end{aligned}
$$

El segundo término de la ecuación anterior es similar al cálculo de una opción Put europea sobre el valor de la empresa con precio de ejercicio $F$, entonces por el Corolario 1.1.1. $D_{0}$ está dado por:

$$
D_{0}=F e^{-r T} \Phi\left(d_{2}\right)+V_{0} \Phi\left(-d_{1}\right) .
$$

Por otro lado de la ecuación (2.1) el valor de mercado $S_{T}$ de la empresa al tiempo $T$ es igual a la diferencia entre el valor de la empresa y el valor de la deuda, entonces

$$
S_{T}=V_{T}-D_{T}=V_{T}-\min \left(V_{T}, F\right)=\max \left(V_{T}-F, 0\right),
$$

y por el Teorema 1.1.1 se tiene que

$$
S_{t}=E^{*}\left[e^{-r(T-t)} \max \left(V_{T}-F, 0\right) \mid \mathscr{F}_{t}\right],
$$

la cual se puede calcular como el valor de una opción call europea. Entonces utilizando el Corolario 1.1.1. se tiene que el valor de una acción al tiempo $t=0$ está dada por:

$$
S_{0}=V_{0} \Phi\left(d_{1}\right)-F e^{-r T} \Phi\left(d_{2}\right) .
$$

En el modelo de Merton se puede caracterizar la probabilidad de incumplimiento de una empresa. 
Proposición 2.1.1. En el modelo de Merton, la probabilidad de incumplimiento de una empresa al tiempo de maduración $T$ es $\Phi\left(-d_{2}\right)$.

Demostración. De la ecuación (2.3) se observa que $V_{T}$ tiene distribución lognormal, es decir,

$$
\ln V_{T} \sim N\left(\ln \left(V_{0}\right)+\left(r-\frac{\sigma_{V}^{2}}{2}\right) T, \sigma_{V}^{2} T\right)
$$

Entonces si al tiempo $T$ se tiene que $V_{T}<F$, la empresa imcumple en pagar el bono y la probabilidad de incumplimiento está dada por:

$$
\begin{aligned}
P\left(V_{T}<F\right) & =P\left(\ln V_{T}<\ln F\right) \\
& =\Phi\left(\frac{\ln \left(F / V_{0}\right)-\left(r-\frac{\sigma^{2}}{2}\right) T}{\sigma_{V} \sqrt{T}}\right) \\
& =\Phi\left(-d_{2}\right) .
\end{aligned}
$$

Sin embargo, para calcular esta probabilidad se necesita conocer el valor de mercado de todos los activos de la empresa así como la volatilidad de los mismos. Desafortunadamente estos valores no son observables directamente en ninguno de los reportes que emiten las empresas y tampoco se pueden desprender de alguno de los indicadores de mercado.

Pero si la empresa que se está analizando cotiza en algún mercado, se puede obtener tanto el valor de mercado del capital accionario, multiplicando el número de acciones en circulación por el precio de mercado de éstas, así como la volatilidad del rendimiento del precio de las acciones. Se demuestra mediante la siguiente proposición que existe una relación directa entre la volatilidad de las acciones $\sigma_{S}$ y la de los activos $\sigma_{V}$.

Proposición 2.1.2. Si el valor de las acciones de la empresa sigue un movimiento browniano geométrico y el valor de los activos de la empresa se describe por la ecuación (2.2), entonces se cumple que:

$$
\sigma_{S}=\frac{V_{t}}{S_{t}} \frac{\partial S_{t}}{\partial V_{t}} \sigma_{V}
$$


Demostración. Sea $S_{t}$ el valor de las acciones de la empresa, entonces

$$
d S_{t}=r_{S} S_{t} d t+\sigma_{S} S_{t} d W_{t}, \quad S_{0}>0 .
$$

Por otra lado de (2.4), $S_{t}$ es igual a valor de una opción call sobre la empresa con precio de ejercicio igual al valor nominal $F$, entonces aplicando Itô a $S_{t}=f\left(t, V_{t}\right)$ se tiene,

$$
d S_{t}=\left[f_{1}\left(t, V_{t}\right)+r V_{t} f_{2}\left(t, V_{t}\right)+\frac{1}{2} \sigma_{S}^{2} V_{t}^{2}\right] d t+\left[\sigma_{V} V_{t} f_{2}\left(t, V_{t}\right)\right] d \tilde{W}_{t},
$$

donde $f_{2}\left(t, V_{t}\right)=\frac{\partial S_{t}}{\partial V_{t}}$. Por lo tanto se debe cumplir que

$$
\begin{aligned}
\sigma_{S} & =\frac{V_{t}}{S_{t}} \frac{\partial S_{t}}{\partial V_{t}} \sigma_{V} \\
& =\frac{V_{t}}{S_{t}} \Phi\left(d_{1}\right) \sigma_{V} .
\end{aligned}
$$

en donde notemos que $\frac{\partial S_{t}}{\partial V_{t}}=\Phi\left(d_{1}\right)$, ver Hull (2009), pág: 352-353.

Con este resultado y el Teorema 2.1.1 se tiene un sistema completo de ecuaciones no lineal, por medio del cual se podrian obtener los valores de $F$ y $\sigma_{V}$. Una vez calculadas estas variables, el diferencial (spread) de rentabilidad o lo que es lo mismo, la diferencia entre el rendimiento a vencimiento de un instrumento riesgoso y una tasa libre de riesgo bajo las mismas condiciones, se obtiene de la siguiente manera:

$$
\begin{aligned}
\text { Diferencial de rentabilidad } & =R(t, T)-r \\
& =-\frac{1}{T} \ln \left(\frac{D_{0}}{F}\right)-r \\
& =-\frac{1}{T} \ln \left(\frac{F e^{-r T} \Phi\left(d_{2}\right)+V_{0} \Phi\left(-d_{1}\right)}{F}\right)-r \\
& =-\frac{1}{T} \ln \left(\Phi\left(d_{2}\right)+\frac{V_{0} \Phi\left(-d_{1}\right)}{F e^{-r T}}\right)
\end{aligned}
$$

donde $d_{1}$ y $d_{2}$ están dados por el Teorema 2.1.1.. Como se puede observar de las fórmulas anteriores, bajo este modelo, el spread de crédito únicamente depende de la madurez del instrumento de deuda y de la volatilidad $\sigma_{V}$ de los activos. 


\subsection{Modelo de Merton con tasa de interés estocástica}

Un primer trabajo que aparece en el estudio de la tasa corta es el modelo de Merton (1973), que proporciona los fundamentos teóricos iniciales para la modelación de tasas de interés en un instante $t$. Una modificación del modelo de Merton es introducir una tasa de interés libre de incumplimiento en donde se mantiene la hipótesis de un sólo instrumento de deuda.

\subsubsection{Modelo de Merton bajo el modelo de Vasicek}

Supongamos que la tasa de interés sigue el modelo de Vasicek, por lo tanto el problema de los precios en el modelo de Merton con un sólo instrumento de deuda es simplemente una aplicación del cambio de numeraria.

Asúmase que bajo una medida martingala $P^{*}$, la dinámica del valor de los activos de la empresa y la tasa corta están dadas por:

$$
\begin{aligned}
d V_{t} & =r_{t} V_{t} d t+\sigma_{V} V_{t}\left(\rho d W_{t}^{1}+\sqrt{1-\rho^{2}} d W_{t}^{2}\right) \\
d r_{t} & =k\left(\theta-r_{t}\right) d t+\sigma_{r} d W_{t}^{1}
\end{aligned}
$$

donde $W_{t}^{1}$ y $W_{t}^{2}$ son movimientos brownianos independientes. Entonces de la Sección 1.4.2 se tiene que el precio al tiempo $t$ de un bono cupón cero libre de incumplimiento con madurez $T$ esta dado por:

$$
B(t, r(t), T)=e^{a(t, T)-r(t) b(t, T)},
$$

donde

$$
b(t, T)=\frac{1-e^{-k(T-t)}}{k},
$$

$\mathrm{y}$

$$
a(t, T)=\frac{1}{k^{2}}(b(t, T)-T+t)\left(k^{2} \theta-\frac{1}{2} \sigma^{2}\right)-\frac{\sigma^{2} b^{2}(t, T)}{4 k} .
$$


Entonces para obtener el valor de las acciones de mercado $S_{t}$ al tiempo $t$, donde la tasa de interés es estocástica, necesitamos calcular:

$$
S_{t}=E^{*}\left[e^{-\int_{t}^{T} r_{s} d s} \max \left(V_{T}-F, 0\right) \mid \mathscr{F}_{t}\right] .
$$

Este cálculo se complica por el hecho de que la variable que se usa para descontar y la función de pago de la opción son variables aleatorias dependientes; y al mismo tiempo que la tendencia del valor de los activos es igual a una tasa de interés estocástica bajo $P^{*}$. Afortunadamente la volatilidad $\sigma_{T}(t)$ de los bonos con madurez $T$ es determinista. Aplicando Itô a el precio del bono se puede demostrar que,

$$
\sigma_{T}(t)=\sigma_{r} b(T, t)
$$

Si definimos $Z_{V, T}(t)=\frac{V_{t}}{B(t, T)}$, la volatilidad de $Z$ también es determinista. Aplicando Itô a $Z$ tenemos,

$$
\begin{aligned}
d Z_{V, T}(t) & =V_{t} d\left(\frac{1}{B(t, T)}\right)+\frac{1}{B(t, T)} d V_{t}+d\left(\frac{1}{B(t, T)}\right) d V_{t} \\
& =H(t) d t+Z_{V, T}\left[\left(b(T-t) \sigma_{r}+\sigma_{V} \rho\right) d W_{t}^{1}+\sigma_{V} \sqrt{1-\rho^{2}} d W_{t}^{2}\right],
\end{aligned}
$$

donde $H(t)$ son los términos de la parte determinista. Por lo tanto la volatilidad de $Z$ esta dada por:

$$
\sigma_{V, T}(t)=\sqrt{\left(\rho \sigma_{V}+\sigma_{r} b(T-t)\right)^{2}+\sigma_{V}^{2}\left(1-\rho^{2}\right)} .
$$

Ahora definiendo

$$
\begin{aligned}
\sum_{V, T}^{2}(T) & =\int_{0}^{T}\left\|\sigma_{V, T}(t)\right\|^{2} d t \\
& =\int_{0}^{T}\left(2 \rho \sigma_{V} \sigma_{r} b(T-t)+\sigma_{r}^{2} b^{2}(T-t)+\sigma_{V}^{2}\right) d t,
\end{aligned}
$$

nos permite enunciar el siguiente teorema:

Teorema 2.2.1. En el modelo de Merton con tasa de interés estocástica, bajo el modelo de Black \& Scholes, los valores del bono riesgoso D y de la acción $S$ al tiempo $t=0$ están 
dados por:

$$
\begin{aligned}
& S_{0}=V_{0} \Phi\left(d_{1}\right)-F B(0, T) \Phi\left(d_{2}\right), \\
& D_{0}=V_{0} \Phi\left(-d_{1}\right)+F B(0, T) \Phi\left(d_{2}\right) .
\end{aligned}
$$

con

$$
\begin{aligned}
\Phi(x) & =\frac{1}{\sqrt{2 \pi}} \int_{-\infty}^{x} e^{-\frac{y^{2}}{2}} d y \\
d_{1} & =\frac{\ln (V / F B(0, T))+\frac{1}{2} \sum_{V, T}^{2}(T)}{\sqrt{\sum_{V, T}^{2}(T)}} \\
d_{2} & =d_{1}-\sqrt{\sum_{V, T}^{2}(T)}
\end{aligned}
$$

Demostración. Ver Karatzas, I. y Shreve, S. (1991), pág. 394-397.

Para evitar la dependencia entre la variable estocástica que se usa para descontar y la función de pago de la opción, supongamos que $\rho \equiv 0$. Por lo tanto por la probabilidad de incumplimiento está dada por $\Phi\left(-d_{2}\right)$.

Al igual que el Modelo de Estructural de Merton, si las empresas que se estan analizando cotizan en algún mercado, se puede calcular el valor de mercado del capital accionario, la volatilidad del rendimiento , así como la correlación entre los dos movimientos brownianos. Se puede demostrar que existe una relación directa entre la volatilidad de las acciones $S$, la de los activos $V$ y la correlación de los movimientos brownianos, entonces tenemos la siguiente proposición:

Proposición 2.2.1. Sea $S$ el valor de las acciones de la empresa, supóngase que $d S_{t}=$ $r_{t} S_{t} d t+\sigma_{S} S_{t}\left(\rho d W_{t}^{1}+\sqrt{1-\rho^{2}} d W_{t}^{2}\right)$, y sea $V$ el valor de los activos de la empresa se describe por la ecuación (2.5), entonces se cumple que:

$$
\sigma_{S}=\frac{V_{t} \frac{\partial S_{t}}{\partial V_{t}} \sigma_{V} \sqrt{1-\rho^{2}}+\frac{\partial S_{t}}{\partial r_{t}} \sigma_{r}}{S_{t} \sqrt{1-\rho^{2}}} .
$$


Demostración. Sea $S_{t}$ el valor de las acciones de la empresa, entonces

$$
d S_{t}=r_{t} S_{t} d t+\sigma_{S} S_{t}\left(\rho d W_{t}^{1}+\sqrt{1-\rho^{2}} d W_{t}^{2}\right), \quad S_{0}>0 .
$$

Por otra lado de (2.9), $S_{t}$ es igual a valor de una opción call sobre la empresa con precio de ejercicio igual al valor nominal $F$, entonces aplicando Itô a $S_{t}=f\left(t, V_{t}, r_{t}\right)$ se tiene,

$$
\begin{aligned}
d f\left(t, V_{t}, r_{t}\right) & =f_{1}\left(t, V_{t}, r_{t}\right) d t+f_{2}\left(t, V_{t}, r_{t}\right) d V_{t}+f_{3}\left(t, V_{t}, r_{t}\right) d r_{t}+\frac{1}{2} f_{2,2}\left(t, V_{t}, r_{t}\right) d V_{t} d V_{t}+ \\
& +f_{2,3}\left(t, V_{t}, r_{t}\right) d V_{t} d r_{t}+\frac{1}{2} f_{3,3}\left(t, V_{t}, r_{t}\right) d r_{t} d r_{t} \\
& =\left[f_{1}\left(t, V_{t}, r_{t}\right)+f_{2}\left(t, V_{t}, r_{t}\right) r_{t} V_{t}+f_{3}\left(t, V_{t}, r_{t}\right) k(\theta-r)\right. \\
& \left.+f_{2,3} \sigma_{r} \sigma_{V} V_{t}\left(\rho^{2}+\sqrt{1-\rho^{2}}\right)+\frac{1}{2}\left\{f_{2,2} \sigma_{V}^{2} V_{t}^{2}\left(1+2 \rho 2 \sqrt{1-\rho^{2}}\right)+f_{3,3}\left(t, V_{t}, r_{t}\right) \sigma_{r}^{2}\right\}\right] d t \\
& +\left[f_{2}\left(t, V_{t}, r_{t}\right) \sigma_{V} V_{t}\left(\rho d W_{t}^{1}+\sqrt{1-\rho^{2}} d W_{t}^{2}\right)+f_{3}\left(t, V_{t}, r_{t}\right) \sigma_{r} d W_{t}^{2}\right]
\end{aligned}
$$

donde $f_{2}\left(t, V_{t}, r_{t}\right)=\frac{\partial S_{t}}{\partial V_{t}}$ y $f_{3}\left(t, V_{t}, r_{t}\right)=\frac{\partial S_{t}}{\partial r_{t}}$. Por lo tanto se debe cumplir que

$$
\begin{aligned}
\sigma_{S} & =\frac{V_{t} \frac{\partial S_{t}}{\partial V_{t}} \sigma_{V} \sqrt{1-\rho^{2}}+\frac{\partial S_{t}}{\partial r_{t}} \sigma_{r}}{S_{t} \sqrt{1-\rho^{2}}} \\
& =\frac{V_{t} \phi\left(d_{1}\right) \sigma_{V} \sqrt{1-\rho^{2}}+\phi\left(d_{2}\right) \sigma_{r}}{S_{t} \sqrt{1-\rho^{2}}}
\end{aligned}
$$

donde $d_{1}$ y $d_{2}$ están dadas por el Teorema 2.2.1.. Con este resultado y el Teorema 2.2.1 se tiene un sistema completo de ecuaciones no lineal, por medio del cual se obtienen los valores de $F$ y $\sigma_{V}$.

\subsubsection{Modelo de Merton bajo el modelo de CIR}

Cuando se asume que la tasa de interés es estocástica, la expresión para la valuación de una opción es en general difícil de obtener, excepto para los procesos gaussianos. Kim y Kunitomo (1999) desarrollaron una simple expresión para aproximar el valor de una opción cuando la tasa de interes sigue un proceso de Itô. Por su parte en Yong (2001) se presenta una adaptación en la aproximación para el caso en donde la tasa de interés no es gaussiana, la cual usaremos en este trabajo. 
Supongamos que la tasa de interés sigue el modelo de CIR y asúmase que bajo una medida martingala $P^{*}$, la dinámica del valor de los activos de la empresa y la tasa corta están dadas por:

$$
\begin{aligned}
d V_{t} & =r_{t} V_{t} d t+\sigma_{V} V_{t}\left(\rho d W_{t}^{1}+\sqrt{1-\rho^{2}} d W_{t}^{2}\right) \\
d r_{t} & =k\left(\theta-r_{t}\right) d t+\sigma_{r} \sqrt{r_{t}} d W_{t}^{1}
\end{aligned}
$$

donde $W_{t}^{1}$ y $W_{t}^{2}$ son movimientos brownianos independientes. Entonces de la sección (1.4.3) se tiene que el precio al tiempo $t=0$ de un bono cupón cero libre de incumplimiento con madurez $T$ esta dado por:

$$
B(t, r(t), T)=a(t, T) e^{-r(t) b(t, T)},
$$

donde

$$
b(t, T)=\frac{2\left(e^{\gamma T}-1\right)}{(\gamma+k)\left(e^{\gamma T}-1\right)+2 \gamma}
$$

$\mathrm{y}$

$$
a(t, T)=\left(\frac{2 \gamma e^{(k+\gamma)(T / 2)}}{(\gamma+k)\left(e^{\gamma T}-1\right)+2 \gamma}\right)^{2 k \theta / \sigma^{2}}
$$

$\operatorname{con} \gamma=\sqrt{k^{2}+2 \sigma^{2}}$.

Bajo la medida neutral al riesgo y utilizando Itô se tiene que

$$
d B(t, r(t), T)=r_{t} B(t, r(t), T) d t-b(t, T) B(t, r(t), T) \sigma \sqrt{r_{t}} d W_{t} .
$$

Utilizando la ecuación (2.13), denotando a $B(t, r(t), T)$ como $B(t, T)$ obtenemos que

$$
\begin{aligned}
\frac{d B(t, T)}{B(t, T)} & =r_{t} d t-\sigma b(t, T) \sqrt{r_{t}} d W_{t} \\
& =\frac{1}{b(t, T)} \ln \left(\frac{a(t, T)}{B(t, T)}\right) d t-\sigma \sqrt{b(t, T) \ln \left(\frac{a(t, T)}{B(t, T)}\right)} d W_{t}
\end{aligned}
$$

Notemos que el porcentaje de volatilidad del precio del bono es una función no 
determinista, pues depende de los niveles actuales del precio del bono. Por lo tanto, para obtener el valor de mercado de la empresa $S_{t}$ al tiempo $t$ dado por:

$$
S_{t}=E^{*}\left[e^{-\int_{t}^{T} r_{s} d s} \max \left(V_{T}-F, 0\right) \mid \mathscr{F}_{t}\right]
$$

se sugiere usar la aproximación propuesta en Yong (2001), la cual se muestra la Proposición 2.2.2.

Proposición 2.2.2. Sea $S$ el valor de las acciones de la empresa, supóngase que la tasa de interés a corto plazo tiene la siguiente la dinámica $d r_{t}=k\left(\theta-r_{t}\right) d t+\sigma_{r} \sqrt{r_{t}} d W_{t}^{2}$, entonces el valor de las acciones de mercado $S_{t}$ al tiempo $t=0$ esta dado por:

$$
\begin{aligned}
S_{0}^{C I R} & =\left[V_{0} \Phi\left(d_{1}\right)-F e^{-\int_{0}^{T} r_{t}^{*} d t} \Phi\left(d_{2}\right)\right] \\
& +\sigma_{r} C_{0}\left[V_{0} \phi\left(d_{1}\right)-F e^{-\int_{0}^{T} r_{t}^{*} d t}\left(\phi\left(d_{2}\right)-\sigma \sqrt{T} \Phi\left(d_{2}\right)\right)\right] \\
& +\sigma_{r} C_{1}\left[d_{2} V_{0} \phi\left(d_{1}\right)-d_{1} F e^{-\int_{0}^{T} r_{t}^{*} d t} \phi\left(d_{2}\right)\right]+o(\delta)
\end{aligned}
$$

donde $\Phi(\cdot)$ es la función de distribución acumulada de una variable aleatoria normal estándar y $\phi(\cdot)$ es su densidad. Por su parte, $r_{t}^{*}$ es una versión determinista de la tasa corta dada por:

$$
r_{t}^{*}=r_{0} e^{-k t}+\theta\left(1-e^{-k t}\right)
$$

y así la versión determinista del factor de descuento tiene la forma:

$$
e^{-\int_{0}^{T} r_{t}^{*} d t}=e^{-\frac{r_{0}-\theta}{k}\left(1-e^{-k T}\right)-\theta T}
$$

Las variables $d_{1} y d_{2}$ están dadas por:

$$
\begin{aligned}
& d_{1}=\frac{1}{\sigma_{V} \sqrt{T}}\left[\log \left(\frac{V_{0}}{F}\right)+\frac{r_{0}-\theta}{k}\left(1-e^{-k T}\right)+\theta T+\frac{\sigma_{V}^{2}}{2} T\right] \\
& d_{2}=d_{1}-\sigma_{V} \sqrt{T} .
\end{aligned}
$$


Por otro lado $C_{0}$ esta representada por:

$$
C_{0}=\frac{1}{\sigma_{V} \sqrt{T}}\left[\frac{\lambda\left(r_{0}-\theta\right)}{k}\left(\frac{1-e^{-k T}}{k}-T e^{-k T}\right)+\frac{\lambda \theta T}{k}\left(1-\frac{1-e^{-k T}}{k}\right)\right] .
$$

Finalmente, $C_{1}$ esta dada por:

$$
C_{1}=-\frac{\rho}{\sigma_{V} T} C_{11}
$$

donde

$$
C_{11}=\frac{2 \sqrt{\theta}\left(\left(1+2 e^{k T}\right) \sqrt{r_{0}}-3 e^{\frac{k T}{2}} \sqrt{r_{0}-\theta\left(1-e^{k T}\right)}\right)+\left(\theta\left(1+2 e^{k T}\right)-r_{0}\right) \psi}{2 e^{k T} k^{2} \sqrt{\theta}},
$$

$y$

$$
\psi=\log \left[\frac{\theta\left(2 e^{k T}-1\right)+r_{0}+2 e^{\frac{k T}{2}} \sqrt{\theta^{2}\left(e^{k T}-1\right)+\theta r_{0}}}{\left(\sqrt{r_{0}}+\sqrt{\theta}\right)^{2}}\right] .
$$

Con la Proposición 2.2.2 y Proposición 2.2.1 se tiene un sistema completo de ecuaciones no lineales, el cual puede ser resuelto por medio del método de Newton Raphson para obtener los valores de $F$ y $\sigma_{V}$.

Para poder analizar el diferencial de rentabilidad en estos modelos como función de los parámetros, se debe centrar el análisis en el efecto que tiene la tasa de interés cuando no existe correlación y el efecto de la correlación en determinados niveles de volatilidad.

\subsection{Modelo de Black y Cox}

Ahora se considera otra extensión del modelo de Merton implementada por Black y Cox en 1975. La idea principal es permitir que el incumplimiento ocurra antes de la maduración del bono. En términos matemáticos, el incumplimiento ocurrirá cuando el nivel del valor de los activos toque la cota inferior, modelado como función del tiempo. 
El modelo de Black y Cox considera un proceso para el valor de los activos, él cual tiene la siguiente dinámica:

$$
d V_{t}=(r-a) V_{t} d t+\sigma V_{t} d W_{t}
$$

en donde se permite el pago continuo de dividendos de radio $a$. La cota de incumplimiento está dada por:

$$
C_{1}(t)=C e^{-\gamma(T-t)}, \gamma \geq 0
$$

Asumamos que el bono emitido por la empresa tiene un principal $F$, tal que $C<F$. Como $V_{t}$ tiene la dinámica descrita por (2.13) entonces $V_{t}=V_{0} e^{(r-a) t-\frac{1}{2} \sigma^{2} t+\sigma W_{t}}$, y el tiempo de incumplimiento $\tau$ está dado por:

$$
\begin{aligned}
\tau & =\inf \left\{0 \leq t \leq T: V_{t}=C_{1}(t)\right\} \\
& =\inf \left\{0 \leq t \leq T: \ln V_{0}+\left((r-a)-\frac{1}{2} \sigma^{2}\right) t+\sigma W_{t}=\ln C-\gamma(T-t)\right\} \\
& =\inf \left\{0 \leq t \leq T:\left(r-a-\frac{1}{2} \sigma^{2}-\gamma\right) t+\sigma W_{t}=\ln C-\gamma T-\ln V_{0}\right\}
\end{aligned}
$$

es decir, el primer tiempo que un movimiento browniano con cierta tendencia alcanza cierto nivel.

En el modelo de Black y Cox la función de pago de los tenedores del bono con madurez $T$ esta dada por:

$$
B\left(V_{T}, T\right)=\min \left(V_{T}, F\right) 1_{\{\tau>T\}},
$$

la cual corresponde con la función de pago usual cuando la cota no se cruza en $[0, T]$. Denotemos a $B^{m}(V, T, F, C, \gamma)$ como el valor al tiempo $t=0$ de la función de pago de un bono con madurez $T$, valor nominal $F$ y cota de incumplimiento $C_{1}(t)$, por lo tanto

$$
B^{m}(V, T, F, C, \gamma)=E\left[e^{-r T} \min \left(V_{T}, F\right) 1_{\{\tau>T\}}\right] .
$$

Si la cota se alcanza antes de la maduración del bono, entonces los tenedores del 
bono se hacen cargo de la empresa, es decir,

$$
B\left(V_{\tau}, \tau\right)=C_{1}(\tau) 1_{\{\tau \leq T\}},
$$

por lo tanto el valor al tiempo $t=0$ de la función de pago de los tenedores del bono cuando se alcanza la cota antes de la fecha de maduración es,

$$
B^{b}(V, T, F, C, \gamma)=E\left[e^{-r T} C_{1}(\tau) 1_{\{\tau \leq T\}}\right] .
$$

Imitando la expresión de la función de pago del bono en el modelo de Merton como la diferencia entre un bono libre de riesgo y un put europeo, notamos que (2.15) se puede reescribir como:

$$
\min \left(V_{T}, F\right) 1_{\{\tau>T\}}=F 1_{\{\tau>T\}}-\max \left(F-V_{T}, 0\right) 1_{\{\tau>T\}} .
$$

Por lo tanto, para el cálculo de $B^{m}$ se pueden emplear algunos resuldados de $o p$ ciones con barrera down and out que se presentaron en los antecedentes. Asumiendo que una cota $C_{1}(t)$ es plana y que la tendencia del activo subyacente es igual a la tasa libre de riesgo bajo la medida neutral al riesgo, la paridad call-put para opciones con barrera nos permite reescribir (2.14) como:

$$
F 1_{\{\tau>T\}}-\max \left(F-V_{T}, 0\right) 1_{\{\tau>T\}}=V_{T} 1_{\{\tau>T\}}-\max \left(V_{T}-F, 0\right) 1_{\{\tau>T\}},
$$

es decir, un contrato de pago del valor de los activos a la madurez si la cota no se alcanza y el precio de un call con barrera. Sea $H(V, T, L)$ el valor de un contrato que paga una unidad a la madurez si el valor de los activos esta por encima de $L$, por lo tanto $H(V, T, L)$ es de la forma:

$$
H(V, T, L)=e^{-r T} \phi\left(\frac{\log (V / L)+\left(r-\frac{1}{2} \sigma^{2}\right) T}{\sigma \sqrt{T}}\right), \quad V>L .
$$

Por el Lema (1.3.2) y la Proposición (1.3.1), el valor $B L$ del pago del bono a la madurez, si la cota es plana al nivel $L$ (corresponde a $\gamma=0$ ) y no existen pagos de dividendos del activo subyacente $(a=0)$, está dada por: 


$$
\begin{aligned}
B L(V, T, F, L) & =L H(V, t, T, L)-L\left(\frac{L}{V}\right)^{\left(\left(2 r / \sigma^{2}\right)-1\right)} H\left(\frac{L^{2}}{V}, t, T, L\right) \\
& +C^{B S}(V, L, T)-\left(\frac{L}{V}\right)^{\left(\left(2 r / \sigma^{2}\right)-1\right)} C^{B S}\left(\frac{L^{2}}{V}, L, T\right) \\
& -C^{B S}(V, F, T)+\left(\frac{L}{V}\right)^{\left(\left(2 r / \sigma^{2}\right)-1\right)} C^{B S}\left(\frac{L^{2}}{V}, F, T\right) .
\end{aligned}
$$

El evento del no incumplimiento se puede ver como:

$$
\{\tau>T\}=\left\{V_{t} e^{-\gamma t}>C e^{-\gamma T}, \text { para todo } t \in[0, T]\right\},
$$

y la función de pago como

$$
\max \left(F-V_{T}, 0\right) 1_{\{\tau>T\}}=e^{\gamma T} \max \left(F e^{-\gamma T}-V_{T} e^{-\gamma T}, 0\right) 1_{\{\tau>T\}} .
$$

Por lo tanto si definimos a $Y_{t}=e^{-\gamma T} V_{t}$, con tendencia $r-a-\gamma$, tenemos que

$$
E^{*}\left[e^{-r T} \max \left(F-V_{T}, 0\right) 1_{\{\tau>T\}}\right]=e^{-a T} E^{*}\left[e^{-(r-a-\gamma) T} \max \left(F e^{-\gamma T}-Y_{T}, 0\right) 1_{\left\{Y_{t}>C e^{-\gamma T\}}\right.}\right],
$$

y la esperanza del segundo término es el valor de una opción call con barrera con activo subyacente $Y$ cuya tendencia bajo la medida neutral al riesgo es $r-a-\gamma$. Entonces el valor al tiempo $t=0$ de la función de pago de un bono con madurez $T$ está dada por:

$$
B^{m}(V, T, F, C, \gamma)=B L\left(V e^{-\gamma T}, T, F e^{-\gamma T}, C e^{-\gamma T}\right)
$$

Ahora para calcular el pago de la opción con barrera (2.14), definamos

$$
u(b, T)=E \int_{0}^{T} C e^{-\alpha s} \varphi_{T, X}(s) d s,
$$

donde $\varphi_{T, X}(s)$ con $s<T$ es el primer tiempo de paro para un movimiento browniano $X$ 
con tendencia y $C$ y $\alpha$ constantes. Utilizando el resultado del Apéndice B, si tomamos

$$
\begin{aligned}
b & =\frac{\log \left(C / V_{0}\right)-\gamma T}{\sigma} \\
\mu & =\frac{r-a-\frac{1}{2} \sigma^{2}-\gamma}{\sigma}
\end{aligned}
$$

y definimos

$$
\tilde{\mu}=\sqrt{\mu^{2}+2 \alpha}
$$

entonces

$$
B^{b}(V, 0, T, C, \gamma, a)=C \frac{e^{b \mu}}{e^{b \tilde{\mu}}}\left(\phi\left(\frac{b-\tilde{\mu} T}{\sqrt{T}}\right)+e^{2 \tilde{\mu} b} \phi\left(\frac{b+\tilde{\mu} T}{\sqrt{T}}\right)\right)
$$

Por lo tanto, se tiene el valor del bono en el modelo de Black-Cox.

Podemos notar que el valor de las acciones en el modelo Black-Cox sin dividendos es una opción call del tipo down-and-out, entonces para determinar el valor de $B^{b}$ se utiliza la diferencia entre el valor de los activos y el valor de las acciones.

Por otro lado si hay pago de dividendos $(a \neq 0), V_{0}$ no sólo es el valor presente de la función de pago de la deuda y las acciones si no también de los flujos de pago de los dividendos, y por lo tanto es necesario el cálculo de $B^{b}$.

Considerando una cota plana al nivel $L$ (corresponde a $\gamma=0$ ), y la no existencia de pagos de dividendos del activo subyacente $(a=0)$, se tiene que el tiempo de incumplimiento $\tau$ está dado por:

$$
\tau=\inf \left\{s \geq t \mid V_{s} \leq C\right\}
$$

Por lo tanto, usando los resultados del Apéndice B se tiene que la probabilidad de incumplimiento del tiempo $t$ al tiempo $T$ es:

$$
P[\tau \leq T \mid \tau>t]=\phi\left(d_{1}\right)+e^{2\left(r-\frac{\sigma_{V}^{2}}{2}\right) \ln \left(\frac{C}{V_{t}}\right) \frac{1}{\sigma_{V}^{2}}} \phi\left(d_{2}\right),
$$


donde

$$
\begin{aligned}
& d_{1}=\frac{\ln \left(\frac{C}{V_{t}}\right)-\left(r-\frac{\sigma_{V}^{2}}{2}\right)(T-t)}{\sigma_{V} \sqrt{T-t}}, \\
& d_{2}=d_{1}-\sigma_{V} \sqrt{T-t} .
\end{aligned}
$$




\section{Capítulo 3}

\section{Modelos de Intensidad}

Los modelos de intensidad también conocidos como modelos de forma reducida, no toman en cuenta la relación entre el incumplimiento y el valor de la empresa de manera explícita. En contraste con lo modelos estructurales, el momento de incumplimiento en los modelos de intensidad no se determina a través del valor de la empresa, sino es el primer salto de un proceso dado exógenamente. Los modelos estructurales establecen un vínculo entre la calidad crediticia de una empresa y las condiciones económicas, financieras de la empresa y así el incumplimiento se genera de forma endógena. Otra diferencia es que las tasas de recuperación, es decir, la recuperación de la empresa como porcentaje del valor de mercado se especifica exógenamente en lo modelos reducidos. En esta sección se presentan los principales resultados asociados a los modelos de intensidad y su utilizada para la valuación de bonos corporativos.

\subsection{Conjuntos de información}

Definamos a $H_{t}$ como un proceso indicador de incumplimientos dado por $H_{t}=I_{\{\tau \leq t\}}$ para todo $t \geq 0$. Notemos que $H_{t}$ es un proceso continuo por la derecha con saltos de 0 a 1 en el tiempo de incumplimiento $\tau$ y que $1-H_{t}=I_{\{\tau>t\}} \equiv H_{t}^{c}$. Sea $\mathscr{X}_{t}$ la filtración generada por el proceso $H$, es decir, $\mathscr{H}_{t}=\sigma\left\{H_{s}, 0 \leq s \leq t\right\}$. Por lo tanto por el proceso estocástico $H_{t}=I_{\{\tau \leq t\}}$ es una submartingala bajo su filtración natural $\mathscr{H}_{t} \mathrm{y}$ si se asume 
diferenciabilidad $^{1}$ se tiene que existe una función positiva $h(t)$ tal que,

$$
H_{t}-\int_{0}^{t} h(s)\left(1-H_{s}\right) d s
$$

es una $\mathscr{H}_{t}$ martingala. Cuando existe un filtración del mercado $\left(\mathscr{G}_{t}\right)_{t \geq 0}$ (lo que en términos generales incluye toda la información que no sea el hecho de incumplimiento o de la sobrevivencia) se tiene un proceso $\lambda_{t}$ que es $\mathscr{G}_{t}$ medible tal que,

$$
H_{t}-\int_{0}^{t} \lambda(s)\left(1-H_{s}\right) d s
$$

es una martingala bajo la "filtración total"

$$
\mathscr{F}_{t}:=\mathscr{H}_{t} \vee \mathscr{G}_{t}
$$

\subsection{Ejemplos de modelos de intensidad}

De manera general los modelos de intensidad suponen una relación entre el tiempo de incumplimiento (condicionado a la filtración $\mathscr{H}$ ) y la filtración del mercado $\mathscr{G}$. Esencialmente se asume que el hecho de que se presente un incumplimiento o no al tiempo $t$ no tiene impacto en la evolución de la filtración del mercado más allá de $t$.

Si el evento de incumplimiento no influye en la tasa de incumplimiento, se puede imaginar que el proceso $H$ es un proceso continuo después del incumplimiento a la misma tasa $\lambda_{t}$. Por lo tanto, $H_{t}=N_{t \wedge \tau}$ donde $N_{t}$ es un proceso de conteo no decreciente y con valores en los enteros tal que $N_{0}=0$. Entonces el tiempo de incumplimiento esta definido por:

$$
\tau=\inf \left\{t \mid N_{t}>0\right\}
$$

En la siguientes secciones, mediante ejemplos, enfocamos el estudio a los casos cuando el proceso de conteo $N_{t}$ admite una intensidad $\lambda_{t}$, y definiendo $\Lambda_{t}^{N}$ la función

\footnotetext{
${ }^{1}$ Ver Grasselli y Hurd (2010).
} 
de intensidad acumulada, es decir,

$$
\Lambda_{t}^{N}=\int_{0}^{t} \lambda_{s} d s
$$

se cumplirá que $N_{t}-\Lambda_{t}^{N}$ es una martingala.

\subsubsection{Procesos Poisson}

Un proceso Poisson con parámetro $\lambda>0$ es un proceso no decreciente con valores en los enteros cuyo valor inicial es $N_{0}=0$, con incrementos independientes y estacionarios con distribución Poisson. Más explícitamente, para todo $0 \leq s<t$ se tiene que

$$
P\left[N_{t}-N_{s}=k\right]=\frac{(t-s)^{k} \lambda^{k}}{k !} e^{-(t-s) \lambda}, k=0,1, \ldots
$$

Notemos que dado (3.2) se tiene que $E\left[N_{t}\right]=\lambda t$ y por lo tanto $\left(N_{t}-\lambda t\right)$ es una martingala. $Y$ así el compensador ${ }^{2}$ de $N_{t}$ es simplemente

$$
\Lambda_{t}^{N}=\lambda t
$$

a partir de la cual se deduce que la constante $\lambda$ es la intensidad para el proceso de Poisson.

El proceso Poisson tiene un número importante de propiedades las cuales hacen que éste proceso se utilice para modelar eventos discretos. Por ejemplo, dado que el proceso Poisson es markoviano se tiene que la ocurrencia de los siguientes $k$ saltos durante algún intervalo de tiempo después de $t$ es independiente de la historia hasta $t$. Además observamos de (3.2) que la probabilidad de un salto durante un intervalo pequeño de longitud $\Delta t$ es aproximadamente $\lambda \Delta t$, y que la probabilidad de que dos o más saltos ocurran al mismo tiempo es cero. Más aún, se puede mostrar que el tiempo de espera entre dos saltos es una variable aleatoria con distribución exponencial y con parámetro $\lambda$. En particular, si se tomamos (3.1) se puede definir el incumplimiento

\footnotetext{
${ }^{2}$ En cálculo estocástico, un compensador es el elemento que le falta a un proceso para que sea martingala.
} 
como el tiempo de arribo del primer salto del proceso $N_{t}$, y entonces la esperanza del tiempo de incumplimiento es $1 / \lambda$ y la probabilidad de sobrevivir después de $t$ años es,

$$
P[\tau>t]=P\left[N_{t}=0\right]=e^{-\lambda t} .
$$

Si asumimos que $P[\tau>t]$ es estrictamente positiva y diferenciable en $t$, se define la función forward de la tasa de incumplimiento como:

$$
\begin{aligned}
h(t) & =-\frac{\partial \log P[\tau>t]}{\partial t} \\
& =\lambda,
\end{aligned}
$$

y así utilizando la regla de Bayes para probabilidad condicional y el hecho de que $\{\tau>$ $t\} \subset\{\tau>s\}$ se deduce que la probabilidad de sobrevivir en $t$ años condicionado a que se sobrevivió hasta $s \leq t$ años es:

$$
\begin{aligned}
P[\tau>t \mid \tau>s] & =\frac{P[\{\tau>t\} \cap\{\tau>s\}]}{P[\tau>s]} \\
& =\frac{P[\tau>t]}{P[\tau>s]} \\
& =e^{-\int_{s}^{t} h(u) d u} \\
& =e^{-\lambda(t-s)} .
\end{aligned}
$$

\subsubsection{Procesos Poisson no homogéneos}

En la práctica, la tasa de incumplimiento cambia en el tiempo ya que la sobrevivencia a diferentes horizontes de tiempo da lugar a diferentes probabilidades de incumplimiento en un intervalo de tiempo pequeño. En este sentido, se obtiene un modelo más realista para modelar la estructura de probabilidades de incumplimiento y así introducir la variabilidad a lo largo del tiempo de la intensidad $\lambda(t)$.

Denotemos a $N_{t}$ como un proceso Poisson no homogéneo, es decir, $N_{t}$ es un proceso no decreciente con valores en los enteros que empieza en $N_{0}=0$ con incrementos 
independientes tal que:

$$
P\left[N_{t}-N_{s}=k\right]=\frac{1}{k !}\left(\int_{s}^{t} \lambda(u) d u\right)^{k} e^{-\int_{s}^{t} \lambda(u) d u},
$$

para alguna función determinista $\lambda(t)$. Observemos que

$$
N_{t}-\int_{0}^{t} \lambda(s) d s
$$

es una martingala y que

$$
\Lambda_{t}=\int_{0}^{t} \lambda(s) d s
$$

es el compensador para $N_{t}$ y por lo tanto $\lambda(t)$ es la intensidad para el proceso Poisson no homogéneo.

Las propiedades del proceso Poisson se extienden de manera natural para un proceso Poisson no homogéneo. Por lo tanto, la probabilidad de un salto sobre un intervalo de tiempo pequeño $\Delta t$ es aproximadamente $\lambda(t) \Delta t$. Más aún el tiempo de espera entre dos saltos es una variable aleatoria con densidad

$$
\lambda(t) e^{-\int_{0}^{t} \lambda(s) d s}, t>0
$$

Por lo tanto, definiendo el incumplimiento como el tiempo de arribo del primer salto del proceso $N_{t}$, se tiene que la probabilidad de sobrevivencia esta dada por:

$$
P[\tau>t \mid \tau>s]=e^{-\int_{s}^{t} \lambda(u) d u}
$$

De la expresión (3.3) se obtiene que $h(t)=\lambda(t)$ correspondiente al proceso Poisson no homogéneo representa la estructura de la tasa de incumplimiento forward la cual puede ser calibrada mediante datos históricos de la misma tasa.

Tanto en el modelo Poisson como en el modelo Poisson no homogéneo, la intensidad $\lambda$ es determinista y por lo tanto sobrevivir a un tiempo $t$ no es la única información relevante para determinar la probabilidad de incumplimiento para el siguiente intervalo $[t, t+\Delta t]$. 
Otros elementos tales como la calificación de crédito y el valor de las acciones de un emisor, o variables macroeconómicas tales como la recesión y los ciclos económicos, proporcionan un flujo adicional de información que debe incorporarse al asignar las probabilidades de incumplimiento. En la siguiente sección se presenta la generalización de modelos basados en la intensidad, la cual permite una intensidad $\lambda_{t}$ estocástica manteniendo algunas propiedades del proceso Poisson.

\subsubsection{Procesos Cox}

Supongamos que toda la información del mercado esta disponible en la economia, excepto por los tiempos de incumplimiento, y dicha información esta representada por la filtración del mercado $\mathscr{G}_{t}$. Por ejemplo, la filtración $\mathscr{G}_{t}$ podría ser generada por un proceso $d$-dimensional $X_{t}$. Además asumamos que todos los factores económicos libres de incumplimiento, incluyendo las tasas de interés libre de riesgo, son adaptables a la filtración $\mathscr{G}_{t}$. Más aún, supongamos que existe un proceso $\lambda_{t}$ no negativo y adaptado a la filtración $\mathscr{G}_{t}$, el cual representa la intensidad estocástica y generalmente esta correlacionado con diferentes componentes del proceso $X_{t}$.

Adicionalmente asumamos que $\mathscr{H}_{t}:=\mathscr{H}_{t}^{N}$ es la filtración generada por el proceso $N_{t}$. Por lo tanto, la filtración total para el modelo es:

$$
\mathscr{F}_{t}=\mathscr{G}_{t} \vee \mathscr{H}_{t}^{N}
$$

Definición 10. El proceso $N_{t}$ es un proceso Cox si, condicionado a la información $\mathscr{G}_{t}$ disponible al tiempo $t, N_{t}$ es un proceso Poisson no homogéneo con intensidad variable en el tiempo $\lambda(s)=\lambda_{s}$ para $0 \leq s \leq t$, esto es:

$$
P\left[N_{t}-N_{s}=k \mid \mathscr{F}_{s} \vee \mathscr{G}_{t}\right]=\frac{\left(\Lambda_{t}-\Lambda_{s}\right)^{k}}{k !} e^{-\left(\Lambda_{t}-\Lambda_{s}\right)},
$$

donde $\Lambda_{t}=\int_{0}^{t} \lambda_{s} d s$. En otras palabra, cada realización del proceso $\lambda$ hasta el tiempo $t$ determina la probabilidad de salto para el proceso $N$ hasta el tiempo $t$. Esta definición algunas veces es conocida como supuesto doblemente estocástico. 
De la definición anterior, para $s<t$ se tiene que

$$
\begin{aligned}
E\left[N_{t}-\Lambda_{t} \mid \mathscr{F}_{s}\right] & =E\left[E\left[N_{t}-\Lambda_{s} \mid \mathscr{F}_{s} \vee \mathscr{G}_{t}\right] \mid \mathscr{F}_{s}\right] \\
& =N_{s}-\Lambda_{s},
\end{aligned}
$$

y por lo tanto el compensador de un proceso Cox es de la forma;

$$
\Lambda_{t}=\int_{0}^{t} \lambda_{s} d s
$$

Por su parte para obtener la probabilidad no condicional de saltos de un proceso Cox, se promedia las realizaciones de la intensidad estocástica usando la expresión de la probabilidad de salto de un proceso no homogéneo para cada realización, es decir,

$$
P\left[N_{t}-N_{s}=k\right]=E\left[\frac{1}{k !}\left(\int_{s}^{t} \lambda_{u} d u\right)^{k} e^{-\int_{s}^{t} \lambda_{u} d u}\right] .
$$

Definiendo el incumplimiento como el arribo del primer salto del proceso $N_{t}$, se tiene que la probabilidad de sobrevivir esta dada por:

$$
P[\tau>t]=E\left[e^{-\int_{0}^{t} \lambda_{u} d u}\right] .
$$

Por lo tanto, utilizando la definición de tasa de incumplimiento, tenemos que

$$
h(t)=-\frac{\partial}{\partial t} \log E\left[e^{-\int_{0}^{t} \lambda_{s} d s}\right] .
$$

De manera general, dado la información condicional $\mathscr{F}_{s}$ disponible hasta el tiempo $s \leq t$, la probabilidad de sobrevivir después de $t$ años es,

$$
P\left[\tau>t \mid \mathscr{F}_{s}\right]=H_{s}^{c} E\left[e^{-\int_{s}^{t} \lambda_{s} d s} \mid \mathscr{G}_{s}\right],
$$

donde $H_{s}^{c}=1-H_{s}$ es el proceso indicador de sobrevivencia, ver Grasselli y Hurd (2010), pág. 61.

Denotando $S P_{s}(t)=P\left[\tau>t \mid \mathscr{F}_{s}\right]$ y asumiendo diferenciabilidad en $t$, se tiene que 
$S P_{s}(t)$ se puede escribir como:

$$
S P_{s}(t)=H_{s}^{c} e^{-\int_{s}^{t} h_{u} d u},
$$

donde

$$
\begin{aligned}
h_{s}(t) & =-\frac{\partial \log S P_{s}(t)}{\partial t} \\
& =-H_{s}^{c} \frac{\partial}{\partial t} \log E\left[e^{-\int_{s}^{t} \lambda_{s} d s} \mid \mathscr{G}_{s}\right],
\end{aligned}
$$

con $h_{s}(t)$ el proceso forward de la tasa de incumplimiento dado toda la información hasta el tiempo $s$, con valor inicial $h_{0}(t)=h(t)$.

Una consecuencia importante de la ecuación (3.4) es que la probabilidad de que la empresa no incumple $(k=0)$ condicionado a la filtración del mercado $\mathscr{G}_{t}$ al tiempo $t$ esta dada por:

$$
\begin{aligned}
P\left[\tau>T \mid \mathscr{G}_{t}\right] & =P\left(N_{T}=0\right) \\
& =E\left[H_{t}^{c} \mid \mathscr{G}_{t]}\right. \\
& =E\left[H_{t}^{c} \mid \mathscr{F}_{0} \vee \mathscr{G}_{t}\right] \\
& =e^{-\int_{0}^{t} \lambda_{u} d u .}
\end{aligned}
$$

La ecuación (3.7) correspondiente a la probabilidad de sobrevivencia, es completamente análoga la fórmula de valuación de un bono cupón cero con un proceso de tasa de interés $\lambda_{s}$. Por lo tanto, el proceso $\lambda_{s}$ puede ser modelado como un proceso de difusión similar al de una tasa de interés. La modelación de la tasa de incumplimiento nos introduce a otros modelos afines a los modelos de intensidad.

\section{Modelos de intensidad Vasicek}

El modelo de intensidad Vasicek es un ejemplo de los modelos afines a los modelos de intensidad, el cual considera un modelo de un factor donde el proceso de intensidad $\lambda_{t}$ sigue la dinámica del modelo Vasicek, es decir,

$$
d \lambda_{t}=k\left(\theta-\lambda_{t}\right) d t+\sigma d W_{t},
$$


donde $k, \theta$ y $\sigma$ son constantes positivas y $W_{t}$ es un movimiento browniano estándar.

$\mathrm{Al}$ igual que para la tasa de interés a corto plazo, los parámetros $k$ y $\theta$ representan el promedio a largo plazo y la tasa de reversión a la media respectivamente para $\lambda_{t}$, mientras que $\sigma$ es el coeficiente de volatilidad. Utilizando la ecuación (3.6) y la Sección 2.2.1 se observa que la probabilidad de sobreviencia en el modelo de intensidad Vasicek esta dada por:

$$
P\left[\tau>t \mid \mathscr{F}_{s}\right]=H_{s}^{c} e^{A(s, t)+B(s, t) \lambda_{s}},
$$

donde

$$
B(s, t)=\frac{1-e^{-k(t-s)}}{k},
$$

$\mathrm{y}$

$$
A(s, t)=\frac{1}{k^{2}}(B(s, t)-t+s)\left(k^{2} \theta-\frac{1}{2} \sigma^{2}\right)-\frac{\sigma^{2} B^{2}(s, t)}{4 k} .
$$

\section{Modelo de intensidad CIR}

Por su parte, el modelo de intensidad CIR es otro ejemplo de los modelos afines a los modelos de intensidad, el cual el proceso de intensidad $\lambda_{t}$ sigue la dinámica del modelo CIR, es decir,

$$
d \lambda_{t}=k\left(\theta-\lambda_{t}\right) d t+\sigma \sqrt{\lambda_{t}} d W_{t},
$$

para $k, \theta$ y $\sigma$ constantes positivas que satisfacen la condición $4 k \theta>\sigma^{2}$. Como es usual, los parámetros $k$ y $\theta$ representan también el promedio a largo plazo y la tasa de reversión a la media respectivamente para $\lambda_{t}$, mientras que $\sigma$ es el coeficiente de volatilidad. Utilizando la Sección 2.2.2 se tiene que la probabilidad de sobrevivencia en el modelo de intensidad CIR tiene esta dada por:

$$
P\left[\tau>t \mid \mathscr{F}_{s}\right]=H_{s}^{c} e^{A(s, t)+B(s, t) \lambda_{s}},
$$


donde

$$
\begin{aligned}
& A(s, t)=\frac{2 k \theta}{\sigma^{2}} \log \left[\frac{2 \gamma e^{\frac{(k+\gamma)(t-s)}{2}}}{2 \gamma+(k+\gamma)\left(e^{(t-s) \gamma}-1\right)}\right] \\
& B(s, t)=\frac{2\left(1-e^{(t-s) \gamma}\right)}{2 \gamma+(k+\gamma)\left(e^{(t-s) \gamma}-1\right)}
\end{aligned}
$$

$\mathrm{y} \gamma^{2}=k^{2}+2 \sigma^{2}$

\subsection{Valuación de bonos con incumplimiento}

Para la valuación de bonos con incumplimiento es necesario introducir una nueva medida $P^{*}$ neutral al riesgo. Dado un modelo de intensidad bajo una medida $P$, no nesariamente se sigue que el modelo sea de intensidad bajo la medida neutral al riesgo $P^{*}$. Más aún, las intensidades $\lambda_{t}^{P}$ y $\lambda_{t}^{P^{*}}$ pueden depender de manera diferente de las variables de estado del modelo, y aunque $\lambda_{t}^{P}=\lambda_{t}^{P^{*}}$ puede ocurrir que

$$
P\left[\tau>T \mid \mathscr{F}_{t}\right]=H_{t}^{c} E\left[e^{-\int_{t}^{T} \lambda_{s} d s} \mid \mathscr{G}_{t}\right]
$$

sea diferente que

$$
P^{*}\left[\tau>T \mid \mathscr{F}_{t}\right]=H_{t}^{c} E^{*}\left[e^{-\int_{t}^{T} \lambda_{s}^{P^{*}} d s} \mid \mathscr{G}_{t}\right]
$$

La aplicación del Teorema de Girsanov a los modelos de intensidad asegura la existencia del cambio de medida $\left.\frac{d P^{*}}{d P}\right|_{\mathscr{F}_{t}}$ para ciertos procesos $\theta_{t}$ y $\eta_{t}$ adaptados tal que si

$$
W_{t}^{P^{*}}=W_{t}+\int_{0}^{t} \theta_{s} d s \text { y } \lambda_{t}^{P^{*}}=\eta_{t} \lambda_{t}
$$

entonces $N_{t}$, un proceso de conteo con intensidad $\lambda$ bajo $P$, tiene intensidad $\lambda^{P^{*}}$ bajo $P^{*}$ y $W_{t}^{P^{*}}$ es un $P^{*}$ movimiento browniano.

Aún definiendo el evento de incumplimiento como el primer salto del proceso de 
conteo $N_{t}$, se tiene que el tiempo de incumplimiento,

$$
\tau=\inf \left\{t>0: N_{t}>0\right\}
$$

tiene una distribución diferente bajo $P^{*}$. Bajo la medida neutral al riesgo $P^{*}$ también se asume que el proceso de conteo $N_{t}$ cumple la propiedad de que

$$
N_{t}-\int_{0}^{t} \lambda_{s}^{P^{*}} d s
$$

sea una $P^{*}$ martingala.

Todas las definiciones anteriores y ejemplos, bajo la nueva medida neutral al riesgo $P^{*}$, son análogas en términos de la intensidad neutral al riesgo $\lambda^{P^{*}}$. En particular,

$$
P^{*}[\tau>t]=E^{*}\left[e^{-\int_{0}^{t} \lambda_{s}^{P^{*}} d s}\right],
$$

la probabilidad de sobrevivencia correspondiente a un proceso Cox.

Considérese un bono cupón cero que inicia en $t$ y madurez $T$, en donde el tiempo de incumplimiento $\tau$ tiene una intensidad $\lambda_{s}$ ( $\lambda_{s}$ la tasa de incumplimiento bajo la medida neutral al riesgo). Además asúmase que existe un proceso de tasa corta $r(s)$ tal que el precio del bono cupón cero sin incumplimiento esta dado por:

$$
B(t, T)=E^{*}\left[e^{-\int_{t}^{T} r_{s} d s} \mid \mathscr{F}_{t}\right] .
$$

Por lo tanto, el precio del bono con riesgo de incumplimiento en el tiempo cero asumiendo que no hay recuperación y que $\tau$ es independiente de $\mathscr{G}_{T}$ esta dado por

$$
\begin{aligned}
\mathfrak{B}(0, T) & =E^{*}\left[e^{-\int_{0}^{T} r_{s} d s} I_{\{\tau>T\}}\right] \\
& =E^{*}\left[E^{*}\left[e^{-\int_{0}^{T} r_{s} d s} I_{\{\tau>T\}} \mid \mathscr{G}_{T}\right]\right] \\
& =E^{*}\left[e^{-\int_{0}^{T} r_{s} d s} E^{*}\left[I_{\{\tau>T\}} \mid \mathscr{G}_{T}\right]\right] \\
& =E^{*}\left[e^{-\int_{0}^{T} r_{s} d s} e^{-\int_{0}^{T} \lambda_{s} d s}\right] \\
& =E^{*}\left[e^{-\int_{0}^{T}\left(r_{s}+\lambda_{s}\right) d s}\right] .
\end{aligned}
$$

La fórmula anterior es general y en la última línea se puede observar el impacto en la 
valuación del bono si $r_{s}$ y $\lambda_{s}$ son dependientes. Por otro lado si se asume independencia entre las dos variables se tiene que

$$
\begin{aligned}
\mathfrak{B}(0, T) & =E^{*}\left[e^{-\int_{0}^{T}\left(r_{s}+\lambda_{s}\right) d s}\right] \\
& =E^{*}\left[e^{-\int_{0}^{T} r_{s} d s}\right] E^{*}\left[e^{-\int_{0}^{T} \lambda_{s} d s}\right] \\
& =B(t, T) P^{*}(\tau>T),
\end{aligned}
$$

es decir, un bono cupón cero con incumplimiento es igual a un bono cupón cero sin incumplimiento por su probabilidad de sobreviviencia bajo la medida neutral al riesgo $P^{*}$. Observemos de (3.7) que

$$
\begin{aligned}
E^{*}\left[I_{\{\tau>T\}} \mid \mathscr{G}_{T}\right] & =P^{*}\left(\tau>T \mid \mathscr{G}_{T}\right) \\
& =e^{-\int_{0}^{T} \lambda_{s} d s} .
\end{aligned}
$$

\subsubsection{Valuación bajo dependencia entre $r_{t}$ y $\lambda_{t}$}

En la valuación de un bono corporativo, el precio puede ser afectado por la dependencia entre el tiempo de incumplimiento $\tau$ y la tasa $r_{t}$, ésta puede surgir de la dependencia que existe entre la tasa de intensidad estocástica $\lambda_{t}$ y la tasa $r_{t}$. Para modelar el comportamiento de la tasa de intensidad $\lambda_{t}$, algunos autores como Embrechts (2008) y Jorda (2010), modelan a $\lambda_{t}$ como un proceso de difusión similar a la tasa de interés. Por lo tanto, considerando el modelo Vasicek para $\lambda_{t}$ y $r_{t}$ se tiene que,

$$
\begin{aligned}
d r_{t} & =k\left(\theta-r_{t}\right) d t+\sigma_{1} d W_{t}^{1} \\
d \lambda_{t} & =\gamma\left(\vartheta-\lambda_{t}\right) d t+\sigma_{2} d W_{t}^{2}
\end{aligned}
$$

donde $k, \theta, \sigma_{1}, c_{1}, \gamma, \vartheta, \sigma_{2} \mathrm{y} c_{2}$ son constantes positivas y $W_{t}^{1} \mathrm{y} W_{t}^{2}$ son movimiento brownianos estándar. La dependencia entre los dos procesos se establece con la correlación entre ambos, es decir, la correlación $\rho d t=d W_{t}^{1} d W_{t}^{2}$ toma valores en el intervalo $[-1,1]$. Y finalmente con ayuda de una discretización de las variables $r_{r}, \lambda_{t}$ y con la ecuación (3.8) se puede valuar el bono con incumplimiento para una empresa. Es importante notar que bajo el supuesto de Vasicek, la tasa de incumplimiento $\lambda_{t}$ puede tomar valores negativos. 
Asumiendo que no existe una tasa de recuperación y que existe dependencia entre la tasa de intensidad estocástica $\lambda_{t}$ y la tasa $r_{t}$, el diferencial de rentabilidad de los modelos de intensidad tiene la siguiente forma general:

$$
\begin{aligned}
\text { Diferencial de rentabilidad } & =\frac{1}{T} \ln \left(E^{*}\left[e^{-\int_{0}^{T}\left(r_{s}+\lambda_{s}\right) d s}\right]\right)-r \\
& =\frac{1}{T} \ln \left(\frac{E^{*}\left[e^{-\int_{0}^{T}\left(r_{s}+\lambda_{s}\right) d s}\right]}{e^{r T}}\right)
\end{aligned}
$$

Para poder aproximar el valor de la esperanza en el diferencial de crédito de estos modelos se utilizó el método de simulación Monte Carlo.

Otra alternativa para valuar un bono corporativo es a través de la tasa de recuperación. En cuanto a la parametrización de la tasa de recuperación, tres especificaciones principales se han adoptado en la literatura. La primera de ellas considera que la tasa de recuperación es una fracción exógena del valor nominal del bono con incumplimiento (Recovery of Face Value, $R F V$ ). Jarrow y Turnbull consideran la tasa de recuperación como una fracción exógena del valor de un equivalente al bono libre de incumplimiento (Recovery of Treasury, RT). Por último, Duffie y Singleton fijan una tasa de recuperación igual a una fracción exógena del valor de mercado de los bonos justo antes del incumplimiento (Recovery of Market Value, RMV). 


\section{Capítulo 4}

\section{Aplicación de los modelos para valuar bonos corporativos de empresas mexicanas}

En los capítulos anteriores, se presentaron algunos modelos de valuación de bonos corporativos entre los cuales están: el modelo de Merton estructural, el modelo de Merton con tasa de interés estocástica, el modelo de Black-Cox y los modelos de intensidad. En el presente capítulo aplicaremos dichos modelos para estimar la probabilidad de incumplimiento de la deuda a corto plazo para el caso de dos empresas Méxicanas. A continuación se describe brevemente el ramo industrial de las dos empresas.

- Cementos Mexicanos(Cemex) es una compañía global de soluciones para la industria de la construcción que ofrece productos de alta calidad y servicios confiables a clientes y comunidades en cuatro continentes. Cemex fue fundada en México en 1906 y desde entonces ha pasado de tener una presencia local, hasta llegar a ser una de las empresas líderes globales en la industria, con más de 50,000 empleados en el mundo. Actualmente, se encuentra estratégicamente posicionada en América, Europa, África, Medio Oriente y Asia. La red de operaciones de Cemex produce, distribuye y comercializa cemento, concreto premezclado, agregados y otros productos relacionados en más de 50 países, y mantiene relaciones comerciales en más de 100 naciones. 
- Grupo Bimbo(Bimbo) es una compañía que fue fundada en 1945 en la Ciudad de México; posteriormete, de 1952 a 1978 se abrieron 12 plantas más, lo que le permitió extender la distribución de sus productos a todo México. Grupo Bimbo inicio su expansión internacional en 1990 y hoy en día se ha convertido en una de las empresas de panificación con mayor presencia a nivel mundial, colocándose como lider en México y en varios paises de latinoamérica. Cuenta con plantas estratégicamente localizadas en Mexico, los EUA, Argentina, Brasil, Chile, Colombia, Costa Rica, el Salvador, Guatemala, Honduras, Nicaragua, Peru, Uruguay, Venezuela, Austria y Republica Checa ${ }^{1}$.

Con ayuda de los modelos estructurales y los modelos de intensidad se pretende modelar la deuda a corto plazo, entendiendo por ello, la deuda emitida con duración menor o igual a un año para Cemex y Bimbo, así mismo estimar su probabilidad de incumplimiento. Cabe aclarar que los resultados que se muestran en este estudio son presentados en unidades monetarias de México, aunque no se descarta la posibilidad de realizar una comparación en términos de dólares utilizando por ejemplo la tasa de T-bill como tasa de interés libre de riesgo.

\subsection{Modelo estructural de Merton}

En el modelo estructural de Merton asume una estructura de capital simple, por lo tanto bajo los supuestos del capítulo anterior lo que necesitamos conocer es el valor de sus activos y el de sus acciones para el tiempo inicial $t=0$; así como tener una estimación de la volatilidad de sus acciones(por ejemplo la volatilidad histórica o la volatilidad usando modelos GARCH), la tasa de interés libre de riesgo del mercado y el horizonte de tiempo para aplicarlo a una empresa. La aplicación de éste modelo se realizó para cada una de las empresas.

En la siguiente tabla se presenta la información financiera básica de las dos empresas para la implementación del modelo de Merton: el valor de los activos $V_{0}$, el valor de las acciones $S_{0}$ y el valor de sus pasivos(corto plazo) $D_{0}$ para diciembre de 2011. Como las empresas que se están analizando cotiza en el mercado mexicano, se puede obtener

\footnotetext{
${ }^{1}$ Fuente: http://www.bmv.com.mx/.
} 
el valor de mercado del capital accionario, multiplicando el número de acciones en circulación por el precio de mercado de éstas. Para obtener el valor de la deuda, se propuso considerar solamente la deuda a corto plazo obtenida del estado de resultados de cada empresa. También se presenta la estimación ${ }^{2}$ de la volatilidad del rendimiento de los precios $\sigma_{S}$ y el rendimiento del activo libre de riesgo ${ }^{3}$. El horizonte de tiempo para él cual se realizaron los cálculos fue $T=1$, que corresponde a un año.

\begin{tabular}{||l|c|c||}
\hline \hline Parámetro & CEMEX & BIMBO \\
\hline $\mathrm{V}_{0}$ & $231,958,518$ & $64,785,160$ \\
\hline $\mathrm{S}_{0}$ & $175,288,070$ & $38,294,997$ \\
\hline $\mathrm{D}_{0}$ & $56,670,448$ & $26,490,163$ \\
\hline$\sigma_{S}$ & 0.60445331 & 0.28176025 \\
\hline \hline
\end{tabular}

Tabla 4.1.Información de las dos empresas para diciembre de 2011 (en miles de pesos). Fuente: Elaboración con datos de Bloomberg.

Utilizando el Teorema 2.1.1 y la Proposición 2.1.2 se tiene un sistema de ecuaciones no lineal que tiene la forma:

$$
\begin{aligned}
& S_{0}-V_{0} \Phi\left(d_{1}\right)+F e^{-r T} \Phi\left(d_{2}\right)=0 \\
& \sigma_{S}-\frac{V_{0}}{S_{0}} \phi\left(d_{1}\right) \sigma_{V}=0 .
\end{aligned}
$$

Resolviendo el sistema no lineal mediante el método de Newton-Raphson ${ }^{4}$, obtenemos $F$ el valor nominal del bono que la empresa deberá pagar en un año, así como el valor de la volatilidad $\sigma_{V}$ de los rendimientos del valor de los activos de la empresa. En la siguiente tabla se muestran la solución del sistema para ambas empresas.

\begin{tabular}{||l|c|c||}
\hline \hline Parámetro & CEMEX & BIMBO \\
\hline $\mathrm{F}$ & $59,145,120$ & $27,639,840$ \\
\hline$\sigma_{V}$ & 0.4569894 & 0.1665506 \\
\hline \hline
\end{tabular}

Tabla 4.2. Resultados del sistema no lineal utilizando Newton-Raphson.

\footnotetext{
${ }^{2}$ La estimación de la volatilidad fue implementada utilizando el modelo $\operatorname{GARCH}(1,1)$ debido a la alta volatilidad de los rendimientos de sus acciones durante el 2011.

${ }^{3}$ Cetes a 28 días según la subasta del último día de cotización del 2011.

${ }^{4}$ Ver Téllez, C. 2010.
} 
En nuestro estudio nos interesa calcular la probabilidad de incumplimiento (PI) y el diferencial de rentabilidad (DC), dichas variables fueron descritas en la Sección 2.1 y correspoden a las ecuaciones (2.5) y (2.6) respectivamente, por lo tanto tenemos los siguientes resultados:

\begin{tabular}{|l|c|c||}
\hline \hline Variable & CEMEX & BIMBO \\
\hline PI & 0.002152958 & 0.000006241376 \\
\hline DC & .03681717 & .0000109822 \\
\hline
\end{tabular}

Tabla 4.3. Probabilidad de incumplimiento(PI) y el Diferencial de crédito(DC).

El valor de los activos para Cemex era de 231,958, 518 millones de pesos, 56, 670, 448 millones de pesos en sus pasivos a corto plazo y una volatilidad estimada del $60.44 \%$ sobre el rendimientos de sus acciones para finales del 2011. Resolviendo el sistema no lineal se obtuvo que el valor de sus pasivos serían de 59, 145, 120 millones de pesos, con una volatilidad estimada de $45.69 \%$ a finales del 2012. Por otro lado, la probabilidad de incumplimiento bajo éste modelo sería de $0.2152958 \%$, mientras que la diferencia entre el rendimiento a vencimiento del Bono y la tasa libre de riesgo ${ }^{5}$ del último día de cotización del 2011 es de $3.68 \%$.

Por otro lado, para Bimbo, el valor de los activos era de $64,785,160$ millones de pesos, 26, 490, 163 millones de pesos en sus pasivos a corto plazo y una volatilidad estimada, menor que la de Cemex, del $28.17 \%$ sobre el rendimientos de sus acciones para finales del 2011. Resolviendo el sistema no lineal se obtuvo que el valor de sus pasivos serían de 27,639, 840 millones de pesos, con una volatilidad estimada de $16.65 \%$ a finales del 2012. Y así mismo, la probabilidad de incumplimiento bajo éste modelo sería de $0.0006241376 \%$, mientras que la diferencia entre el rendimiento a vencimiento del Bono y la tasa libre de riesgo es $0.00109822 \%$.

Una observación importante sobre las dos empresas es que la probabilidad que Cemex incumpla en sus obligaciones es mayor que la probabilidad que Bimbo lo haga, esto se debe a que en los ultimos años Cemex con la intensión de ampliar sus activos realizó varias inversiones que no cumplieron con las espectativas de sus empresarios y

\footnotetext{
${ }^{5}$ En algunos estudios se utiliza la tasa de interés interbancaria de equilibrio (TIIE).
} 
por lo tanto tuvieron que pagar varias deudas. Los problemas de Cemex comenzaron cuando compró en el 2007 la productora australiana de materiales de construcción Rinker, lo que triplicó su deuda y aumentó su presencia en el debilitado mercado de Estados Unidos. Por último, en la siguiente gráfica se muestra el diferencial de rentabilidad o spread para ambas empresas en términos de puntos base $\mathrm{e}^{6}$.

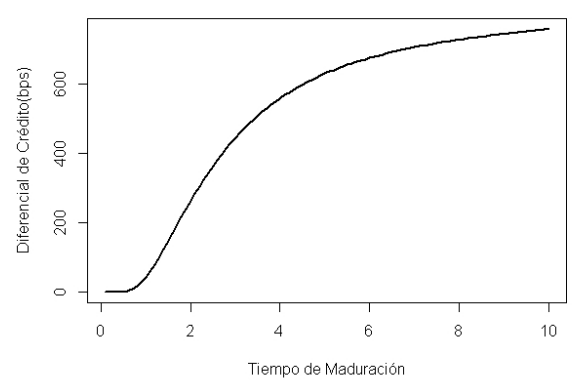

(a) Diferencial de rentabilidad CEMEX

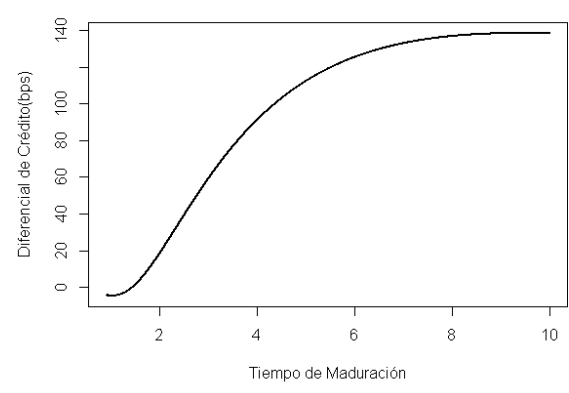

(b) Diferencial de rentabilidad BIMBO.

Figura 4.1: Gráfica del diferencial de rentabilidad como función del tiempo de maduración en el modelo clásico de Merton.

Se puede observar que Bimbo produce menores diferenciales de rentabilidad que Cemex, lo cual se puede traducir en el hecho que Cemex ofrece un exceso de rentabilidad mayor respecto a los bonos de gobiernos, que el que ofrece Bimbo. Es natural pensar que dado que Cemex tiene un mayor apalancamiento que Bimbo, los inversionistas demanden una prima de riesgo mayor.

\subsection{Modelo de Merton con tasa de interés estocástica}

El modelo de Merton con tasa de interés estocástica es una modificación del modelo original de Merton; en nuestro estudio se supone que la tasa de interés sigue el modelo de Vasicek descrito en el capítulo de los antecedentes, por lo tanto el problema de los precios en el modelo de Merton con un sólo instrumento de deuda es simplemente una aplicación del cambio de numeraria. El modelo de Vasicek afirma que la tasa de interés

\footnotetext{
${ }^{6}$ Un punto base es equivalente a $.01 \%$.
} 
tiene la siguiente dinámica:

$$
d r(t)=k(\theta-r(t)) d t+\sigma_{r} d W(t), k>0 .
$$

Dado que los parámetros $k, \theta$ y $\sigma_{r}$ no pueden ser establecidos a priori, se realizó una estimación de los parámetros mediante el método de Máxima Verosimilitud visto en la Sección 1.5.1 utilizando como base la subasta semanal de la tasa de rendimiento de Cetes de 28 días para el año 2011 con un total de 52 datos, el resultado de dichas estimaciones para las dos empresas se muestra en la siguiente tabla:

\begin{tabular}{||l|c||}
\hline \hline Variable & Estimación \\
\hline $\mathrm{k}$ & 0.1335929214 \\
\hline$\theta$ & 0.0425694346 \\
\hline$\sigma_{r}$ & 0.0005868121 \\
\hline \hline
\end{tabular}

Tabla 4.4. Estimación de parámetros para el modelo Vasicek.

La estimación del parámetro $\theta$ nos indica que para valores grandes de $t$ (largo plazo), la tasa de interés a corto plazo se estacionará alrededor de $4.256 \%$ a una velocidad de ajuste del $13.35 \%$. Con esto se puede comprobar la propiedad de reversión a la media del modelo de Vasicek. Aunque el modelo de Vasicek tiene la desventaja económica de que puede producir tasas negativas, la elección de este modelo sobre la tasa de interés se basó en la Sección 2.2.2., y en el hecho de que bajo el modelo CIR no existe una fórmula cerrada para valuar opciones y por tanto calcular el valor de las acciones $S_{t}$.

El factor (numerario) que se utilizó para descontar el valor de los activos $V_{t}$ fue un bono cupón cero, por lo tanto el precio al tiempo $t=0$ del bono cupón cero libre de incumplimiento que paga una unidad monetaria a la fecha de maduración $T=1$ fue de 0.958400019 .

La información financiera básica de las dos empresas para la implementación del modelo de Merton con tasa de interés estocástica es la misma que la del modelo de Merton clásico, así mismo se propone estimar la correlación $\rho$ de la Proposición 2.2.1 mediante los datos históricos de la tasa de interés a corto plazo y los precios de las acciones de Cemex y Bimbo obteniendo una $\rho_{C E M E X}=.059$ y $\rho_{B I M B O}=-.359$. En este 
caso utilizando el Teorema 2.2.1. y la Proposición 2.2.1 se tuvo que resolver un nuevo sistema no lineal que tiene la siguiente forma:

$$
\begin{aligned}
S_{0}-V_{0} \Phi\left(d_{1}\right)+F B(0, T) \Phi\left(d_{2}\right) & =0 \\
\sigma_{S}-\frac{V_{t} \frac{\partial S_{t}}{\partial V_{t}} \sigma_{V} \sqrt{1-\rho^{2}}+\frac{\partial S_{t}}{\partial r_{t}} \sigma_{r}}{S_{t} \sqrt{1-\rho^{2}}} & =0
\end{aligned}
$$

en donde

$$
\begin{aligned}
\Phi(x) & =\frac{1}{\sqrt{2 \pi}} \int_{-\infty}^{x} e^{-\frac{y^{2}}{2}} d y \\
d_{1} & =\frac{\ln (V / F B(0, T))+\frac{1}{2} \sum_{V, T}^{2}(T)}{\sqrt{\sum_{V, T}^{2}(T)}} \\
d_{2} & =d_{1}-\sqrt{\sum_{V, T}^{2}(T)}
\end{aligned}
$$

y $\sum_{V, T}^{2}(T)$ está dado por la ecuación (2.10).

Resolviendo el sistema no lineal mediante el método de Newton-Raphson, se obtuvo el valor nominal del bono que la empresa deberá pagar en un año. En la siguiente tabla se muestran la solución del sistema para ambas empresas.

\begin{tabular}{||l|c|c||}
\hline \hline Variable & CEMEX & BIMBO \\
\hline $\mathrm{F}$ & $58,639,450$ & $28,242,030$ \\
\hline$\sigma_{V}$ & 0.461123 & 0.170679 \\
\hline \hline
\end{tabular}

Tabla 4.5. Resultados del sistema no lineal utilizando Newton-Raphson.

Por último se calculó la probabilidad de incumplimiento para las dos empresas y se obtuvo que Cemex tiene una probabilidad de incumplimiento de $0.3754482 \%$, mientras que Bimbo una de 8.368084E - 05\%. Las probabilidades obtenidas en éste modelo son menores a las que se presentadas en el modelo de Merton clásico, esto conforme a la teoría expuesta en la Sección 2.2.1.

El valor de los activos, los pasivos a corto plazo y la volatilidad estimada para los dos empresas fueron los mismos que en el modelo clásico de Merton. Pero resolviendo 
el sistema no lineal de éste modelo se obtuvo que para Cemex el valor de sus pasivos serían de 58,639, 450 millones de pesos con una volatilidad estimada de $46.11 \%$; y para Bimbo el valor de sus pasivos serían de 28,242,030 millones de pesos con una volatilidad estimada de $17.06 \%$ a finales del 2012.

Al igual que el modelo de Merton clásico, Bimbo produce menores spreads de crédito que Cemex, lo cual implica que Cemex, en el modelo de Merton con tasa de interés estocástica, también ofrece un exceso de rentabilidad mayor del que ofrece Bimbo sobre los bonos del gobierno. Otro factor importate que tiene un impacto en los spreads es la probabilidad de incumplimiento, ya que dicha probabilidad afecta el riesgo por impago de cada empresa y por tanto en los diferenciales. La siguiente gráfica muestra el diferencial de rentabilidad como función del tiempo de maduración.

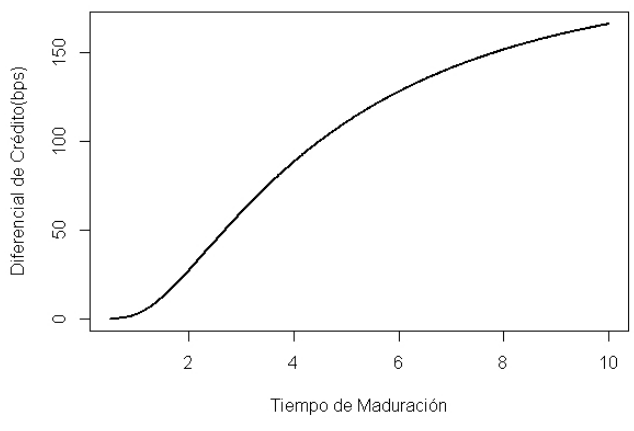

(a) Diferencial de rentabilidad CEMEX

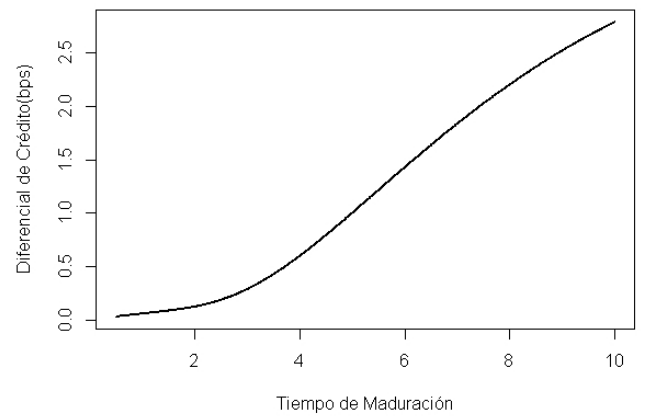

(b) Diferencial de rentabilidad BIMBO.

Figura 4.2: Gráfica del diferencial de rentabilidad como función del tiempo de maduración en el modelo de Merton con tasa de interés estocástica.

Es importante notar que las diferencias (spread) obtenidas en éste modelo son mucho menores a las obtenidas en la Figura 4.1 que corresponden al modelo de Merton clásico.

Con el objetivo de comparar los resultados anteriores obtenidos bajo el modelo Vasicek, se propone modelar la deuda a corto plazo para ambas empresas utilizando la aproximación propuesta en Yong (2001)(Ver [33]) para el modelo CIR. Por lo tanto, con ayuda de la Proposión 2.2.1 y la Proposición 2.2.2 se estimó el valor de $F$ y $\sigma_{V}$, los resultados se muestran en la Tabla 4.6: 


\begin{tabular}{||l|c|c||}
\hline \hline Variable & CEMEX & BIMBO \\
\hline $\mathrm{F}$ & $59,129,990$ & $27,639,840$ \\
\hline$\sigma_{V}$ & 0.1507742 & 0.1381239 \\
\hline \hline
\end{tabular}

Tabla 4.6. Resultados del sistema no lineal utilizando Newton-Raphson. Aproximación de Yong (2001) para modelo CIR.

Una observación importante en la Tabla 4.5 y Tabla 4.6 es que el valor de la deuda de ambas empresas es similar tanto para el modelo de Vasicek como para el modelo de CIR. Por lo tanto, al menos para Cemex y Bimbo, la aproximación propuesta en Yong (2001) para el modelo CIR es similar a la forma cerrada del modelo Vasicek.

\subsection{Modelo de Black y Cox}

Tanto el modelo de Merton como el de Black y Cox pertenecen a la familia de los modelos estructurales, que determinan la probabilidad de que una empresa alcance un nivel de insolvencia (antes del tiempo de madurez de la deuda), tomando como referencia el valor de mercado de la misma. Estos modelos establecen un vínculo entre la calidad de crédito de la empresa y su condición financiera. El modelo de Black y Cox extiende el enfoque de Merton al caso en que la empresa puede alcanzar un nivel de insolvencia en cualquier momento previo a la fecha de expiración de la deuda.

Para determinar el pago del bono a la madurez, denotado por $B L$ en la ecuación (2.18), si la cota $L$ es plana (corresponde a $\gamma=0$ ) y no existen pagos de dividendos del activo subyacente $(a=0)$, se utilizan los resultados de la Tablas 4.1 y Tabla 4.2, obtenidas para el modelo de clásico de Merton. Antes de determinar el valor del pago del bono a la madurez $B L$, se necesita estimar el valor de la barrera inferior $L$ para ambas empresas, por lo tanto usando la condición $V>L$ y realizando 100,000 simulaciones sobre el proceso

$$
V_{t}=V_{0} e^{\left(r-\frac{\sigma_{V}^{2}}{2}\right) t+\sigma_{V} \tilde{W}_{t}}
$$


se estimaron cotas inferiores $L$ con cierto nivel de confianza ${ }^{7}$ para cada empresa,

\begin{tabular}{||l|c|c||}
\hline \hline Cota & CEMEX & BIMBO \\
\hline L(1\%) & $121,156,336$ & $51,914,584$ \\
\hline L(5\%) & $217,399,165$ & $61,696,045$ \\
\hline \hline
\end{tabular}

Tabla 4.7. Estimación del valor de la cota $L$ con un 1\% y 5\% de confianza.

Así es posible determinar el precio $B^{m}$ al tiempo $t=0$, que corresponde al valor al tiempo $t=0$ de la función de pago de un bono con madurez $T$, valor nominal $F$ y cota de incumplimiento $L$ en el modelo de Black-Cox en la ecuación (2.15) y sabiendo que:

$$
B^{m}(V, T, F, L, \gamma)=B L\left(V e^{-\gamma T}, T, F e^{-\gamma T}, L e^{-\gamma T}\right) .
$$

Dado que no hay pago de dividendos, el valor al tiempo $t=0$ de la función de pago de los tenedores del bono cuando se alcanza la cota antes de la fecha de maduración ,$B^{b}$, se determina como la diferencia entre el valor de mercado los activos y el valor de mercado de las acciones. La siguiente tabla muestra el valor del bono en el modelo Black y Cox, clasificado en $B^{m}$ y $B^{b}$ para las dos empresas.

\begin{tabular}{||l|c|c||}
\hline \hline Bono & CEMEX & BIMBO \\
\hline $\mathrm{B}^{m}(1 \%)$ & $55,842,460$ & $28,741,333$ \\
\hline $\mathrm{B}^{m}(5 \%)$ & $13,989,535$ & $11,056,982$ \\
\hline $\mathrm{B}^{b}(1 \%)$ & $56,670,448$ & $26,490,163$ \\
\hline $\mathrm{B}^{b}(5 \%)$ & $56,670,448$ & $26,490,163$ \\
\hline \hline
\end{tabular}

Tabla 4.8. Valor del bono con $1 \%$ y $5 \%$ de confianza en el modelo Black-Cox.

En la tabla anterior podemos observar que el valor del bono en el modelo Black y Cox depende en gran medida del valor de la cota inferior $L$, la cual representa el nivel de incumplimiento. Por lo tanto para Cemex y Bimbo, la cota representada por el $1 \%$ de

\footnotetext{
${ }^{7}$ El nivel de confianza es el porcentaje de trayectorias simuladas que al tiempo $t=T$ estuvieron por debajo de la cota $L$.
} 
los valores $V_{T}$ menor $L$ es la que modela el valor de bono de manera similar al modelo de Merton.

Por su parte la probabilidad de incumplimiento para CEMEX es 0.1871314, mientras que para BIMBO es de 0.1447629 calculadas en la ecuación (2.19). Esta probabilidades de incumplimiento también son sensibles al valor de la cota inferior $L$, es decir, dependen de las simulaciones para la obtención de la cota inferior.

Una comparación importante entre los modelos de valuación de bonos corporativos es el diferencial de rentabilidad, pues cuando no hay pago de dividendos el modelo de Black-Cox muestra menores diferenciales en comparación con el modelo clásico de Merton ${ }^{8}$. En la Figura 4.3 se puede observar que el diferencial de rentabilidad, tanto para Cemex como para Bimbo, muestra menores diferenciales o spreads en modelo Black-Cox que en el modelo de Merton.

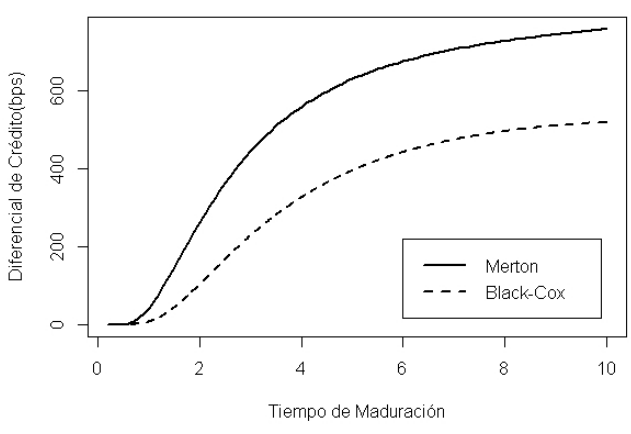

(a) Diferencial de rentabilidad CEMEX

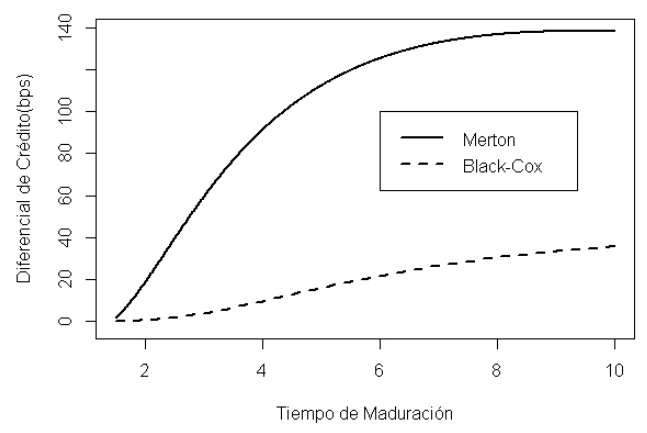

(b) Diferencial de rentab ilidadBIMBO.

Figura 4.3: Comparativo del diferencial de rentabilidad para el modelo de Merton y Black-Cox.

Con los resultados obtenidos en los tres modelos estructurales antes estudiados, se puede concluir que Cemex tiene mayores diferenciales de rentabilidad que Bimbo, lo cual es consecuencia que Cemex tiene mayor apalancamiento que Bimbo. Los diferenciales se traducen en un mayor exceso de rentabilidad del bono de Cemex sobre el bono de Bimbo, ambos respecto a los bonos gubernamentales.

\footnotetext{
${ }^{8}$ Ver Lando (2004).
} 


\subsection{Modelos de Intensidad}

En los modelos de intensidad, una de las principales dificultades es una correcta estimación de los parámetros de la tasa de interés y de la tasa de incumplimiento. Debido a que en el mercado no se tiene un bono corporativo similar al utilizando en este estudio, no fue posible estimar la probabilidad de incumplimiento de manera implícita. Por lo tanto, algunos autores sugieren utilizar la tasa publicada por las agencias calificadoras referentes al incumplimiento.

La analogía de la valuación de bonos corporativos en los modelos de intensidad con respecto a los modelos estructurales facilita el cálculo de dichos modelos. Sin embargo, la intepretación de los resultados debe hacerse de manera cuidadosa. En los modelos de intensidad el incumplimiento no se determina a través del valor de la empresa sino es el primer salto de un proceso exógeno, esto puede conducir a resultados erróneos ${ }^{9}$.

En esta sección nos enfocamos a la valuación de bonos corporativos bajo el supuesto de dependencia entre el tiempo de incumplimiento $\tau$ y la tasa de interés a corto plazo $r_{t}$. Dicha dependencia puede ser resultado de la dependencia que existe entre la intensidad estocástica $\lambda_{t} \mathrm{y}$ la tasa de interés $r_{t}$. Como vimos en la Sección 3.2, es natural modelar a $\lambda_{t}$ como un proceso de difusión similar a la tasa de interés a corto plazo. Por ejemplo, considerando el modelo de Vasicek para $\lambda_{t}$ y $r_{t}$ se tiene el siguiente modelo:

$$
\begin{aligned}
d r_{t} & =k\left(\theta-r_{t}\right) d t+\sigma_{1} d W_{t}^{1} \\
d \lambda_{t} & =\gamma\left(\vartheta-\lambda_{t}\right) d t+\sigma_{2} d W_{t}^{2}
\end{aligned}
$$

con $k, \theta, \sigma_{1}, c_{1}, \gamma, \vartheta, \sigma_{2}$ y $c_{2}$ son constantes positivas y $W_{t}^{1}$ y $W_{t}^{2}$ son movimiento brownianos estándar.

Dado que los parámetros $k, \theta$ y $\sigma_{1}$ correspondientes a la tasa de interés a corto plazo no pueden ser establecidos a priori, se realizó una estimación de los mismo mediante el método de Máxima Verosimilitud, descrito en la Sección 1.5, utilizando como base el promedio de la tasa de Cetes de 28 días para cada año del 1990 al 2011; mientras que para los parámetros $\gamma, \vartheta$ y $\sigma_{2}$, que corresponden a la tasa de incumplimiento, se uso el

\footnotetext{
${ }^{9}$ Ver ejemplo en Embrechts(2008).
} 
mismo método sobre la tasa de incumplimiento ${ }^{10}$ públicada por la agencia calificadora Standard \& Poors en el 2011. Dichas estimaciones se muestra en la Tabla 4.9.

Recordemos que el valor de $\theta$ y $\vartheta$ son los valores a largo plazo de las tasa de interés y la tasa de incumplimiento, mientras que la rapidez de convergencia esta representada por $k$ y $\gamma$ respectivamente para cada modelo.

\begin{tabular}{||l|l||}
\hline \hline Variable & Estimación \\
\hline $\mathrm{k}$ & 0.46469613 \\
\hline$\theta$ & 0.14625526 \\
\hline$\sigma_{1}$ & 0.26392880 \\
\hline$\gamma$ & 1.03658918 \\
\hline$\vartheta$ & 0.01654979 \\
\hline$\sigma_{2}$ & 0.02544968 \\
\hline \hline
\end{tabular}

Tabla 4.9. Estimación de parámetros bajo el modelo Vasicek.

Valuando bajo depencencia entre $r_{t} \mathrm{y} \lambda_{t}$, el precio del bono corporativo para Cemex y Bimbo depende de la correlación entre los dos movimiento brownianos estándar, $W_{t}^{1}$ y $W_{t}^{2}$. La correlación, definida por $\rho d t=d W_{t}^{1} d W_{t}^{2}$, modela la dependencia entre la tasa de interés $r_{t}$ y la tasa de incumplimiento $\lambda_{t}$, $\operatorname{con} \rho \in[-1,1]$.

Utilizando la fórmula (3.8) para $T=1$ (un año), y con los parámetros obtenidos en la Tabla 4.8 se discretizó las variables $r_{t}$ y $\lambda_{t}$ en el intervalo $[0,1] \operatorname{con} \Delta t=\frac{1}{1 * 100}$ para calcular los precios de los bonos corporativos para ambas empresas con 100,000 simulaciones y con $\rho \in[-1,1]$. En Ekstrom y Tysk (2007) se muestran ciertas propiedades de los parámetros de algunos procesos de difusión, afirmando que el precio del bono es creciente respecto a la volatilidad del proceso subyacente.

Por lo tanto, en la siguiente figura se muestran dos gráficas en donde se podría justificar la tendencia del precio del bono para el caso de Cemex.

\footnotetext{
${ }^{10}$ Ver Estudio anual de incumplimiento y análisis de transición de calificaciones de los sectores corporativos, de servicios financieros y finanzas públicas, para el caso mexicano. Página electrónica: www.standardandpoors.com
} 


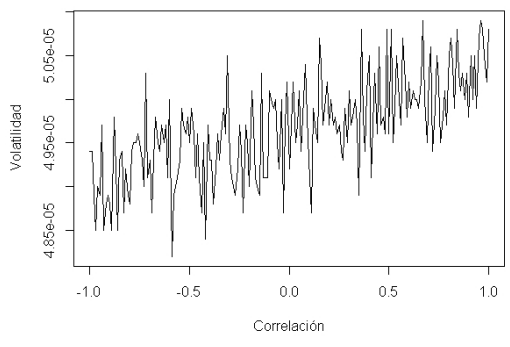

(a) $\operatorname{Var}\left(\int_{0}^{1}\left(r_{s}+\lambda_{s}\right) d s\right)$

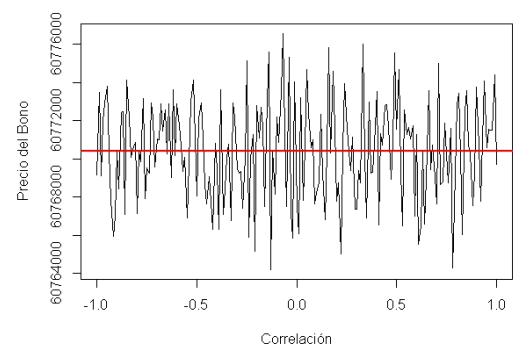

(b) Precio del bono.

Figura 4.4: Evolución del precio del bono corporativo para Cemex como función de la correlación.

En la Figura 4.4 (a), se observa que una correlación negativa entre $r_{t}$ y $\lambda_{t}$ disminuye la volatilidad de la suma, mientras que una correlación positiva aumenta la volatilidad, y por lo tanto la volatilidad es creciente cuando la correlación también crece, observando que la escala de la Figura 4.4 (a) es relativamente pequeña. El incremento en el precio del bono se debe al incremento en la volatilidad de la suma de los procesos. Sin embargo, en la Figura 4.4 (b) no se puede observar de manera clara la tendencia positiva del precio del bono como función de la correlación.

Por su parte para la valuación del bono corporativo de Bimbo se obtuvieron resultados similares, los cuales se muestran en la siguientes figura:

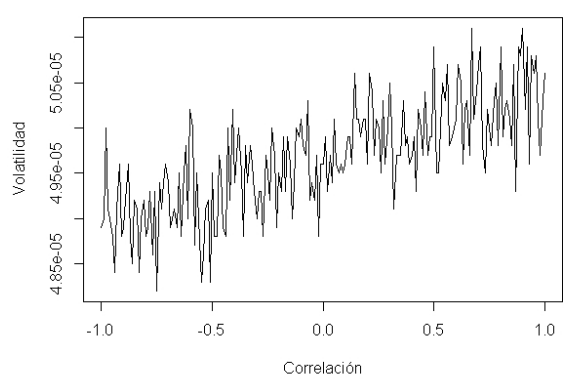

(a) $\operatorname{VaR}\left(\int_{0}^{1}\left(r_{s}+\lambda_{s}\right) d s\right)$

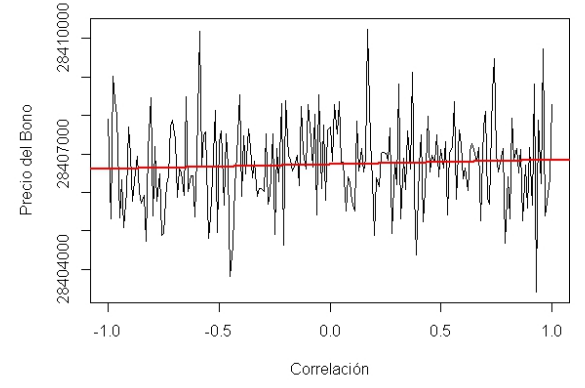

(b) Precio del bono.

Figura 4.5: Evolución del precio del bonos corporativo para Bimbo como función de la correlación.

En las Figuras 4.4 y 4.5 se presenta la evolución del precio del bono de Bimbo como función de la correlación $\rho$. Para el caso de Bimbo, se observa una tendencia positiva 
en la evolución del precio del bono como función de la correlación, a diferencia del bono de Cemex. Por lo tanto, no se puede confirmar las aseveraciones de Ekstrom y Tysk (2007) para el precio de un bono.

Una de las diferencias en la aplicación de los modelos estructurales y el modelo de intensidad, fue que para el segundo hubo necesidad de ajustar el período de análisis. El ajuste se realizó debido a que la información sobre tasas de incumplimiento, proporcionada por Standard \& Poors, se muestra de forma anual mientras que la tasa de interés con periodicidad de cada 28 días. De esta manera el cálculo de la correlación entre $W_{t}^{1}$ y $W_{t}^{2}$, la cual modela la dependencia entre $r_{t}$ y $\lambda_{t}$, se pudo determinarse directamente de los datos. Recordando que $\rho d t=d W_{t}^{1} d W_{t}^{2}$ y realizando una discretización del modelo descrito por la ecuación (4.1) se tiene que,

$$
\begin{aligned}
r_{t} & =\alpha_{1}+\beta_{1} r_{t-1}+\eta_{t}^{1} \\
\lambda_{t} & =\alpha_{2}+\beta_{2} \lambda_{t-1}+\eta_{t}^{2}
\end{aligned}
$$

$\operatorname{con} \eta_{t}^{1} \sim N\left(0, \sigma_{1}^{2} \Delta t\right), \eta_{t}^{2} \sim N\left(0, \sigma_{2}^{2} \Delta t\right)$ y $\Delta t=T / n$

Por lo tanto, dado que la correlación es invariante bajo transformaciones lineales o afines, se tiene que los residuales de $r_{t}$ y $\lambda_{t}$, y los dos brownianos tienen la misma correlación. A continuación se muestra gráficamente la dependencia que existe entre las dos tasas y entre sus residuales.

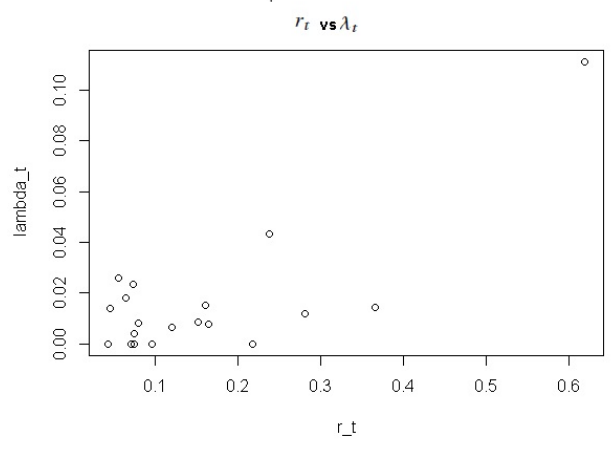

(a) $r_{t}$ vs. $\lambda_{t}$

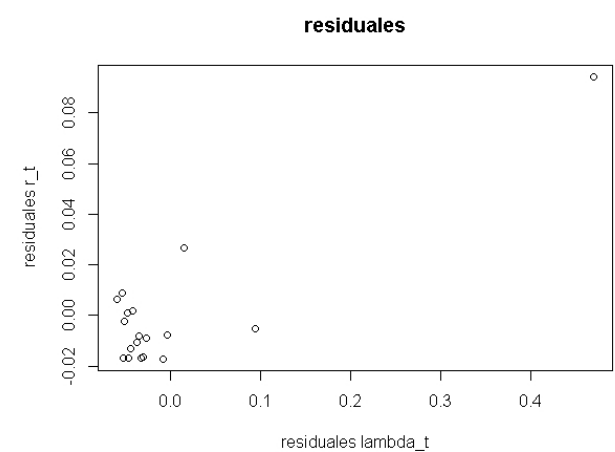

(b) Residuales de $r_{t}$ y $\lambda_{t}$.

Figura 4.6: Gráficas de la tasa de interés a corto plazo vs tasa de incumplimiento, y sus respectivos residuales. 
La Figura anterior muestra de manera gráfica una correlación alta, en donde el resultado de la correlación para ambas empresas es $\rho=.83$, y así el precio promedio de los bonos correspondientes a la misma correlación son:

\begin{tabular}{||l|c|c||}
\hline \hline Precio & CEMEX & BIMBO \\
\hline Bono & $60,745,012$ & $28,895,201$ \\
\hline \hline
\end{tabular}

Tabla 4.10. Estimación del precio del bono en base a la correlación de $W_{t}^{1} \mathrm{y} W_{t}^{2}$.

En la tabla anterior se muestra que el precio del bono con incumplimiento para Cemex, bajo el modelo de intensidad es del 60,745,012 millones de pesos; mientras que el precio del bono de Bimbo con incumplimiento es de 28,895,201 millones de pesos. En general, el modelo de intensidad generó valores más grandes para los bonos que los modelos estructurales. Por lo tanto, la estimación de la deuda para finales del 2012 para ambas empresas es mayor bajo los modelos de intensidad, dicho resultado se observa en la siguiente tabla:

\begin{tabular}{||l|c|c||}
\hline \hline Modelo & CEMEX & BIMBO \\
\hline Merton & $59,145,120$ & $27,639,840$ \\
\hline M. Estocástico & $58,639,450$ & $28,242,030$ \\
\hline Black-Cox & $55,842,460$ & $28,741,333$ \\
\hline Intensidad & $60,745,012$ & $28,895,201$ \\
\hline \hline
\end{tabular}

Tabla 4.11. Comparativo entre los modelos estructurales e Intensidad.

Por último, para determinar el diferencial de crédito para los modelos de intensidad, se decidió tomar el valor de los bonos con incumplimiento y utilizando la fórmula (3.9) se obtuvo que Cemex tiene un diferencial de rentabilidad o spread de $0.137 \%$ al final del período de análisis, mientras que Bimbo tiene una diferencial del $0.129 \%$ en el mismo período. Al igual que en los modelos estructurales, en este modelo Cemex presenta menores diferenciales (spreads) de rentabilidad que Bimbo.

En términos generales, los modelos aplicados en este estudio son congruentes con las calificaciones públicadas para Cemex y Bimbo por algunas agencias calificadoras, 
Capítulo 4. Aplicación de los modelos para valuar bonos corporativos de empresas mexicanas

tales como Standard \& Poors o Moody's. Actualmente dichas agencias calificadoras asignan una calificación de $B+$ a Cemex, mientras que $B B B$ a Bimbo en la emisión de Eurobonos, lo cual implica que Bimbo tiene una mejor solvencia (capacidad de pago de la deuda emitida: bonos, obligaciones, etc.) que Cemex. 


\section{Capítulo 5}

\section{Conclusiones}

En los mercados financieros es de vital importancia la correcta y oportuna medición de los distintos tipo de riesgos que se puedan presentar. Uno de los más importantes es el riesgo de crédito. De manera particular, en el mercado de bonos corporativos, el riesgo de crédito o insolvencia se entiende como el riesgo derivado del incumplimiento de los compromisos por parte del emisor del bono, entendiendo como insolvencia la posibilidad de no recibir el importe íntegro prometido o de que se produzca un retraso en el pago del mismo. Con el objetivo de modelar la deuda de Cemex y Bimbo a corto plazo y estimar la probabilidad de incumplimiento, se recurrió al uso de algunos modelos de crédito. Dentro de los cuales estan los modelos estructurales y los modelos de intensidad.

Los modelos estructurales se caracterizan por utilizar información específica de la empresa. En ellos, se supone que el valor de la deuda que posee la empresa viene dado por el valor de una opción sobre los activos de la empresa. Y así, el riesgo de crédito se deriva de la relación entre el valor de la empresa y el valor de su deuda, definiendo la insolvencia del emisor en el momento en que el valor de la empresa es inferior a una barrera preestablecida que depende directamente del nivel de endeudamiento de la empresa en cuestión. En general estos tipo de modelos tienden a subestimar la probabilidad de incumplimiento y por tanto las medidas de riesgo. Dentro de las principales dificultades que se presentan en los modelos estructurales están: obtener el valor de la empresa y determinar cuál es la barrera bajo la cual la empresa se considera incapaz de hacer frente a sus compromisos. 
Por su parte, en los modelos de forma reducida o de intensidad se trabaja directamente con la información del mercado. En general, el riesgo de crédito se modela a partir de la información implícita en los precios del mercado, en los diferenciales de rentabilidad y, en ocasiones en la matriz de probabilidades de transición de calidad crediticia. Dado que existen muchas variantes de los modelos de intensidad, en este trabajo solamente se presenta una breve introducción a dichos modelos asumiendo dependencia entre la tasa de interés a corto plazo y la tasa de incumplimiento. Por lo tanto, la implementación de este tipo de modelos es análoga a la presentada en los modelos de estructurales. Sin embargo, la intensidad el incumplimiento no se determina a través del valor de la empresa sino es el primer salto de un proceso exógeno. Otros estudios más avanzados sobre los modelos de intensidad enfocan la teoría asumiendo que existe una tasa de recuperación.

Uno de los principales supuestos en la tesis para la implementación de los modelos estructurales fue asumir que toda la estructura de deuda de la empresa esta concentrada en un único bono cupón cero, lo que implica además en el caso del modelo de Merton clásico, que la insolvencia solo puede producirse en un solo momento, es decir, a la fecha de vencimiento de la deuda. Con la intensión de corregir la restricción del incumplimiento en el modelo de Merton clásico, se recurrió al modelo de Black y Cox en el cual se asumió que no hay pago de dividendos y se introdujo una barrera de incumplimiento. En nuestro estudio se realizaron simulaciones del valor de los activos de las empresas para determinar la barrera para cada empresa con un cierto nivel de confianza. El valor de la barrera depende en gran medida de la tendencia que tengan los procesos de manera particular. Otra modificación que frecuentemente se hacen sobre el modelo de Merton clásico es suponer cierta dinámica en la tasa de interés. Los modelos que se utilizaron para modelar la tasa a corto plazo fueron el modelo Vasicek y el modelo CIR; sabemos que el modelo CIR ofrece en general características deseables en una tasa, pero en la teoría sobre la valuación de una opción, el modelo de Vasicek tiene forma cerrada mientras que para el modelo CIR se recurrió a la aproximación presentada por Yong (2001). Los valores de las deudas a finales del 2012 para Cemex y Bimbo obtenidos para la tasa de interés con Vasicek son similares a los resultados bajo la aproximación del modelo CIR. Una de las principales características en estos modelos son que todos presentan probabilidades de incumplimiento y diferenciales 
de rentabilidad relativamente bajos, a causa de que la forma del incumplimiento puede predecirse y no puede ocurrir en forma sorpresiva, ver [31].

En el caso de los modelos de intensidad hubo necesidad modificar el período de historia, debido a la disponibilidad de datos para estimar los parámetros. Como la información disponible de las tasas de incumplimiento es anual, se tuvo que modificar el período de analisis sobre la tasa de Cetes a 28 días a una tasa promedio anual, correspondiente a los mismos años disponibles para la tasa de incumplimiento. De esta manera se pudo inferior la correlación que existe entre la tasa de interés y la tasa de incumplimiento, y así la dependencia que existe entre las dos tasas. En Ekstrom y Tysk (2007) se afirma que el precio del bono es una función creciente respecto a la correlación, lo cual de manera particular no se pudo observar de manera clara para las empresas analizadas.

Entre los resultados más importantes de este trabajo se encuentra que los modelos de intensidad modelan un mayor valor de la deuda para Cemex y Bimbo que los modelos estructurales. Este resultado pudo ser causado por la modificación en la história de las tasas. En todos los modelos, Cemex tiene una probabilidad de incumplimiento mayor que Bimbo, esto se debe a que en los ultimos años Cemex con la intensión de ampliar sus activos realizó varias inversiones que no cumplieron con las espectativas de sus empresarios y por lo tanto tuvieron que pagar varias deudas.

Finalmente, es importante aclarar que este trabajo no pretende analizar la totalidad de las metodologías relacionadas con la modelación de la deuda de una empresa mexicana, solo intenta estudiar los modelos más populares y ver las dificultades que se presentan en su implementación en el mercado mexicano. Así mismo, es importante mencionar que en los modelos de intensidad se presentaron los resultados más importantes relacionados con la valuación de bonos corporativos. Dado que en general los métodos estructurales subestiman la probabilidad de incumplimiento según el mercado, se sugiere estudiar modelos híbridos para intentar replicar lo observado en el mercado mexicano. 


\section{Apéndice A}

\section{Algunos resultados de probabilidad}

\section{A.1 Teorema de Girsanov}

El teorema de Girsanov construye explícitamente una medida de probabilidad que permite transformar un movimiento browniano con tendencia en un movimiento browniano sin tendencia.

Algunos resultados importantes para la demostración del Teorema de Girsanov se muestran a continuación:

Teorema A.1.1. (Lévy). Sea $\left(W_{t}\right)_{\{0 \leq T\}}$ un proceso sobre $(\Omega, \mathscr{F}, P)$ adaptado a la filtración $\mathscr{F}_{t}$, tal que

1. Las trayectorias de $W_{t}$ son continuas.

2. Wes martingala

3. La varianción cuadrática del proceso $W$, la cual se denotará por $[W, W]_{t}=t$.

Entonces W es un movimiento browniano.

Demostración. Ver Karatzas, I. y Shreve, S. (1991), pág. 168-169. 
Sea $(\Omega, \mathscr{F}, P)$ un espacio de probabilidad y $Z$ un variable aletoria no negativa tal que $E[Z]=1$, entonces se define una nueva medida de probabilidad $P^{*}$ por:

$$
P^{*}(A)=\int_{A} Z(w) d P(w), \text { para todo } A \in \mathscr{F} \text {. }
$$

Para cualquier $X$ variable aleatoria, tenemos que

$$
E^{*}[X]=E[X Z]
$$

y si $P(Z>0)=1$, entonces $P$ y $P^{*}$ coinciden en los conjuntos de medida cero, y (A.2) tiene la siguiente forma:

$$
E[X]=E^{*}\left[\frac{X}{Z}\right]
$$

Se dice que $Z$ es la derivada de Radon-Nikodýn de $P^{*}$ con respecto a $P$, y se denota por

$$
Z=\frac{d P^{*}}{d P}
$$

De manera formal, supóngase que se tiene un espacio de probabilidad $(\Omega, \mathscr{F}, P)$ y una filtración $\left(\mathscr{F}_{t}\right)_{\{0 \leq t \leq T\}}$; y además supongasé que existe $Z$ una variable no negativa casi seguramente tal que $E[Z]=1$ y defina $P^{*}$ como en (A.1) entonces se define la derivada de Radon-Nikodýn como:

$$
Z(t)=E\left[Z \mid \mathscr{F}_{t}\right]
$$

Dado que para $s \leq t$

$$
\begin{aligned}
E\left[Z(t) \mid \mathscr{F}_{s}\right] & =E\left[E\left[Z \mid \mathscr{F}_{t}\right] \mid \mathscr{F}_{s}\right] \\
& =E\left[Z \mid \mathscr{F}_{s}\right]=Z(s),
\end{aligned}
$$

se tiene que el proceso de derivada de Radon-Nikodýn es una martingala.

Además ésta derivada tiene ciertas propiedades: 
Lema A.1.1. Sea $Y$ un variable aleatoria $\mathscr{F}_{t}$ medible, entonces

$$
E^{*}[Y]=E[Y Z(t)]
$$

Demostración. Sea $0 \leq t \leq T$, usando (A.2), y propiedades de esperanza condicional tenemos

$$
E^{*}[Y]=E[Y Z]=E\left[E\left[Y Z \mid \mathscr{F}_{t}\right]\right]=E\left[Y E\left[Z \mid \mathscr{F}_{t}\right]\right]=E[Y Z(t)] .
$$

Lema A.1.2. Sea $Y$ un variable aleatoria $\mathscr{F}_{t}$ medible y sea $0 \leq s \leq t \leq T$, entonces

$$
E^{*}\left[Y \mid \mathscr{F}_{s}\right]=\frac{1}{Z(s)} E\left[Y Z(t) \mid \mathscr{F}_{s}\right] .
$$

Demostración. Sea $0 \leq s \leq t \leq T$, notemos que $\frac{1}{Z(s)} E\left[Y Z(t) \mid \mathscr{F}_{s}\right]$ es medible, entonces para $A \in \mathscr{F}_{s}$, se tiene

$$
\begin{aligned}
\int_{A} \frac{1}{Z(s)} E\left[Y Z(t) \mid \mathscr{F}_{s}\right] d P^{*} & =E^{*}\left[1_{\{A\}} \frac{1}{Z(s)} E\left[Y Z(t) \mid \mathscr{F}_{s}\right]\right] \\
& =E\left[1_{\{A\}} E\left[Y Z(t) \mid \mathscr{F}_{s}\right] \mid \mathscr{F}_{t}\right] \\
& =E\left[E\left[1_{\{A\}} Y Z(t)\right] \mid \mathscr{F}_{s}\right] \\
& =E\left[1_{\{A\}} Y Z(t)\right] \\
& =E^{*}\left[1_{\{A\}} Y\right] \\
& =\int_{A} Y d P^{*}
\end{aligned}
$$

Teorema A.1.2. (Girsanov). Sea $W_{t}, 0 \leq t \leq T$, un movimiento browniano sobre un espacio de probabilidad $(\Omega, \mathscr{F}, P)$ y una filtración $\left(\mathscr{F}_{t}\right)_{\{0 \leq t \leq T\}}$ para este movimiento browniano. Sea $\theta(t)$ un proceso previsible. Defina

$$
Z(t)=e^{-\int_{0}^{t} \theta(u) d W(u)-\frac{1}{2} \int_{0}^{t} \theta^{2}(u) d u,}
$$


$y$

$$
\tilde{W}(t)=W(t)+\int_{0}^{t} \theta(u) d u
$$

y suponga que

$$
E\left[\int_{0}^{T} \theta^{2}(u) Z^{2}(u) d u\right]<\infty
$$

Sea $Z=Z(T)$, entonces $E[Z]=1$ y el proceso $\tilde{W}_{t}$ es un movimiento browniano bajo la medida de probabilidad $P^{*}$.

Demostración. Notemos que el proceso $\tilde{W}$ comienza en cero y es continuo. Dado que $\int_{0}^{t} \theta(u) d u$ tiene variación cuadrática finita, entonces $[\tilde{W}, \tilde{W}]_{t}=[W, W]_{t}=t$.

Ahora veamos que $\tilde{W}$ es martingala bajo $P^{*}$, pero antes demostremos que $Z(t)$ es martingala bajo $P$. Aplicando el lema de Itô a $f(x)=e^{x}$ con

$$
X(t)=-\int_{0}^{t} \theta(u) d W(u)-\frac{1}{2} \int_{0}^{t} \theta^{2}(u) d u,
$$

tenemos que:

$$
\begin{aligned}
d Z(t) & =e^{X(t)}\left(-\theta(t) d W(t)-\frac{1}{2} \theta^{2}(t) d t\right)+\frac{1}{2} e^{X(t)} \theta^{2} d t \\
& =-\theta(t) Z(t) d W(t) .
\end{aligned}
$$

Integrando, tenemos que

$$
Z(t)=Z(0)-\int_{0}^{t} \theta(u) Z(u) d W(u)
$$

Como las integrales de Itô son martingala, entonces $Z(t)$ es una martingala. En particular, $E[Z]=E[Z(T)]=Z(0)=1$.

Dado que $Z(t)$ es una martingala y que $Z=Z(T)$, se tiene que

$$
Z(t)=E\left[Z(T) \mid \mathscr{F}_{t}\right]=E\left[Z \mid \mathscr{F}_{t}\right] \text {, para } 0 \leq t \leq T
$$

Esto muestra que $Z(t)$ es la derivada de Radon-Nikodýn de $P^{*}$ respecto a $P$. 
Ahora se demuestra que $\tilde{W}(t) Z(t)$ es martingala bajo $P^{*}$. Aplicando la regla de integración por partes para el proceso estocástico $\tilde{W}(t) Z(t)$ tenemos:

$$
\begin{aligned}
d(\tilde{W}(t) Z(t)) & =\tilde{W}(t) d Z(t)+Z(t) d \tilde{W}(t)+d \tilde{W}(t) d Z(t) \\
& =-\tilde{W}(t) \theta(t) Z(t) d W(t)+Z(t) d W(t)+Z(t) \theta(t) d t \\
& +(d W(t)+\theta(t) d t)(-\theta(t) Z(t) d W(t)) \\
& =(-\tilde{W}(t) \theta(t)+1) Z(t) d W(t) .
\end{aligned}
$$

Dado que la expresión final no tiene término $d t$, el proceso $\tilde{W}(t) Z(t)$ es una martingala bajo $P^{*}$. Ahora dado $0 \leq s \leq t \leq T$, por el Lema (A.1.2) y la propiedad de martingala de $\tilde{W}_{t} Z_{t}$, se tiene que

$$
E\left[\tilde{W}_{t} \mid \mathscr{F}_{t}\right]=\frac{1}{Z(s)} E\left[\tilde{W}_{t} Z(t) \mid \mathscr{F}_{s}\right]=\frac{1}{Z(s)} \tilde{W}_{s} Z(s)=\tilde{W}_{s},
$$

es decir, $\tilde{W}_{s}$ es una martingala bajo $P^{*}$. Por lo tanto usando el Teorema de Lévy, se concluye que $\tilde{W}_{t}$ es un movimiento browniano bajo la medida de probabilidad $P^{*}$.

\section{A.2 Teoremas fundamentales de valuación de activos.}

Teorema A.2.1. (Primer Teorema Fundamental). Si un modelo de mercado tiene una medida de probabilidad neutra al riesgo, entonces no hay arbitraje.

Demostración. Ver Karatzas, I. y Shreve, S. (1991), pág. 231.

Definición 11 (mercado completo). Un modelo de mercado libre de arbitraje se dice que es completo si cualquier opción es replicable, es decir, si se puede construir una cartera admisible cuyo valor en cualquier tiempo $t \in[0, T]$ coincida con el valor de la opción.

Teorema A.2.2. (Segundo Teorema Fundamental). Consideremos un modelo de mercado que tiene un medida de probabilidad neutra al riesgo. Entonces el modelo de mercad es completo si y sólo si la medida neutra al riesgo es única.

Demostración. Ver Karatzas, I. y Shreve, S. (1991), pág. 232. 


\section{Apéndice B}

\section{Algunos resultados de movimientos brownianos}

Sea $W$ un movimiento browniano estándar sobre un espacio de probabilidad $(\Omega, \mathscr{F}, P)$. Si $X=\sigma W_{t}+\mu t$, entonces $X$ es un movimiento browniano con tendencia $\mu$ y volatilidad $\sigma$. En está sección denotaremos $X$ como un $(\mu, \sigma)$ movimiento browniano; y se desarrollan los resultados para cotas inferiores las cuales son usadas para modelar el incumplimiento.

Consideremos un $(\mu, \sigma)$ movimiento browniano que empieza en $X_{0}=x_{0}$, y la cota inferior está dada por el valor 0 . Por lo tanto $x_{0}$ es la distancia hacia la cota inferior. Sea $m_{t}$ el mínimo del proceso $X$ hasta el tiempo $t$,

$$
m_{t}=\inf \left\{X_{s}: 0 \leq s \leq t\right\}
$$

Entonces usando Harrison(1990), la distribución conjunta del proceso y su mínimo está dada por:

$$
P^{x_{0}}\left(X_{t}>x, m_{t}>y\right)=\Phi\left(\frac{x_{0}-x+\mu t}{\sigma \sqrt{t}}\right)-e^{\frac{-2 \mu\left(x_{0}-y\right)}{\sigma^{2}}} \Phi\left(\frac{2 y-x_{0}-x+\mu t}{\sigma \sqrt{t}}\right), 0 \leq y \leq x .
$$

Denotando a $\tau$ como el primer tiempo de paso para cero,

$$
\tau:=\inf \left\{X_{t}=0: X_{0}=x_{0}, t \geq 0\right\}
$$


Así mismo la probabilidad del evento $\left\{m_{t}>y\right\}$ se calcula con (B.1) para $x=0$ entonces tenemos:

$$
P(\tau>t)=\phi\left(\frac{x_{0}+\mu t}{\sigma \sqrt{t}}\right)-e^{\frac{-2 \mu\left(x_{0}\right)}{\sigma^{2}}} \phi\left(\frac{2 y-x_{0}+\mu t}{\sigma \sqrt{t}}\right), t \geq 0
$$

Por lo tanto, la distribución asociada al primer paso es $P(\tau \leq t)=1-P(\tau>t)$. Cuando la tendencia es positiva, existe una probabilidad positiva de nunca alcanzar la cota; mientras que cuando la tendencia es cero, la cota es alcanzada con probabilidad uno en un tiempo finito pero el tiempo de paro tiene esperanza infinita. ${ }^{1}$.

Para el modelo de Black y Cox, en donde los bonos tienen madurez finita y ocurrencia de incumplimiento cuando se alcanza una cota (inferior), se necesita cálcular el valor presente del pago de una unidad cuando se alcanza la cota antes de la fecha de maduración $T$. Sea $(\mu, 1)$ movimiento browniano que empieza en $X_{0}=0$, y

$$
\tau_{b}=\inf \left(t \geq 0: X_{t}=b\right)
$$

entonces podemos calcular

$$
\begin{aligned}
V(b, T) & =E^{\mu}\left[e^{-\alpha \tau_{b}} 1_{\tau_{b} \leq T}\right] \\
& =\int_{0}^{T} e^{-\alpha \tau_{b}} P^{\mu}\left(\tau_{b} \in d s\right),
\end{aligned}
$$

donde $P^{\mu}\left(\tau_{b} \in d s\right)$ es la densidad de $\tau_{b}$. Si $\alpha=0$ entonces $V(b, T)=P^{\mu}\left(\tau_{b} \leq T\right)$, la cual tiene una expresión análitica conocida. Cuando $\alpha \neq 0$ el truco es poner a $\alpha$ como parte de la tendencia del movimiento browniano y definir una nueva expresión $P^{\tilde{\mu}}\left(\tau_{b} \leq T\right)$ con una diferente $\tilde{\mu}$.

De Karatzas y Shreve (1991), pág.112-113 sabemos que

$$
P^{\mu}\left(\tau_{b} \leq T\right)=\int_{0}^{T} e^{\mu b-\frac{1}{2} \mu^{2} s} P^{0}\left(\tau_{b} \in d s\right)
$$

\footnotetext{
${ }^{1}$ Ver Karatzas y Shreve (1991), pág. 112-113.
} 
Por lo tanto

$$
\begin{aligned}
V(b, T) & =\int_{0}^{T} e^{-\alpha s} e^{\mu b-\frac{1}{2} \mu^{2} s} P^{0}\left(\tau_{b} \in d s\right) \\
& =\frac{e^{\mu b}}{e^{b \sqrt{\mu^{2}+2 \alpha}}} \int_{0}^{T} e^{b \sqrt{\mu^{2}+2 \alpha}-\frac{1}{2}\left(\mu^{2}+2 \alpha\right) s} P^{0}\left(\tau_{b} \in d s\right) \\
& =\frac{e^{\mu b}}{e^{b \sqrt{\mu^{2}+2 \alpha}}} P^{\sqrt{\mu^{2}+2 \alpha}}\left(\tau_{b} \leq T\right)
\end{aligned}
$$

Del modelo de Black y Cox recordemos que el tiempo de incumplimiento $\tau$ está dado por:

$$
\begin{aligned}
\tau & =\inf \left\{0 \leq t \leq T: \sigma W_{t}+\left(r-a-\frac{1}{2} \sigma^{2}-\gamma\right) t=\log \left(C / V_{0}\right)-\gamma T\right\} \\
& =\inf \left\{0 \leq t \leq T: W_{t}+\frac{\left(r-a-\frac{1}{2} \sigma^{2}-\gamma\right) t}{\sigma}=\frac{\log \left(C / V_{0}\right)-\gamma T}{\sigma}\right\}
\end{aligned}
$$

entonces si se toma

$$
\begin{aligned}
b & =\frac{\log \left(C / V_{0}\right)-\gamma T}{\sigma} \\
\mu & =\frac{r-a-\frac{1}{2} \sigma^{2}-\gamma}{\sigma}
\end{aligned}
$$

y si definimos $\tilde{\mu}=\sqrt{\mu^{2}+2 \alpha}$, se puede reescribir $P^{\tilde{\mu}}\left(\tau_{b} \leq T\right)$ como:

$$
P^{\tilde{\mu}}\left(\tau_{b} \leq T\right)=\phi\left(\frac{b-\tilde{\mu} t}{\sqrt{t}}\right)+e^{2 \tilde{\mu} b} \phi\left(\frac{b+\tilde{\mu} t}{\sqrt{t}}\right)
$$

y así

$$
V(b, T)=\frac{e^{\mu b}}{e^{b \sqrt{\mu^{2}+2 \alpha}}} P^{\tilde{\mu}}\left(\tau_{b} \leq T\right) .
$$




\section{Bibliografía}

[1] Ball, C. y Torous, W. 1996. Unit Roots and the estimation of Interest Rate Dynamics. Journal of Empirical Finance, 3:215-238.

[2] Björk, T. 2004. Arbitrage theory in continuos time. Segunda Edición. Oxford University Press.

[3] Black, F. y Cox, J. 1975. Valuating Corporate Securities: Some Effects of Bond Indenture Provisions. Journal of Finance, 31, 2, 351-367.

[4] Black, F. y Scholes, M. 1973. The pricing of options and corporate liabilities. Journal of Political Economy 81, 3, 673-654.

[5] Brennan, M., and E. Schwartz, 1980. Analyzing Convertible Bonds., Journal of Financial and Quantitative Analysis, 15, 907-929.

[6] Brigo, D. y Mercurio, F. 2006. Interest Rate Models-Theory and Practice. Segunda Edición. Springer.

[7] Cox, J.C., Ingersoll, J.E. y Ross, S.A. 1985. A Theory of Term Structure of Interest Rates. Econometrica, 53, 385-407.

[8] Das, S. y P. Tufano, 1995. Princing Credit Sensitive Debt When Interest Rates, Credit Ratings, and Credit Spreads Are Stochastic. Journal of Financial Engineering, 5, 161-198.

[9] Ekström, E. y Tysk, J. 2008. Convexity theory for the term structure equation. Finance Stochastic 12, 117-147. 
[10] Elizalde, A. 2012. Credit Risk Models I: Default Correlation in Intensity Models. Frank Fabozzis Encyclopedia of Financial Models, Chp. 22.

[11] Elizalde, A. 2012. Credit Risk Models I: Structural Models. Frank Fabozzis Encyclopedia of Financial Models, Chp. 23.

[12] Embrechts, P., A.J. McNeil y R. Frey. 2005. Quantitative Risk Management. Princeton Series in Finance, USA.

[13] Feller, W. 1951. Two Singular Diffusion Problems. Annals of Mathematics, Vol. 54, No. 1, pp. 173-182.

[14] Grasselli, M. R. y Hurd, T. R. 2010. Credit Risk Modeling. Working paper.

[15] Harrison, J. 1990. Brownian motion and stochastic flow systems. Malabar, FL: Robert E. Krieger.

[16] Heath, D., Jarrow, R. y Morton, A. 1992. Bond Princing and the Term Structure of Interest Rates: A New Methodology for Contigent Claims Valuation. Econometrica, Vol. 60, 77-105.

[17] Hull, J. 2009. Options, Futures and Others Derivatives. Octava Edición Englewood Cliffs, NJ: Prentice Hall.

[18] Jarrow, R., and S. Turnbull, 1995. Pricing Derivatives on Financial Securities Subject to Default Risk. Jorunal of Finance, 50, 53-86.

[19] Jarrow, R., D. Lando, and S. Turnbull, 1997. A Markov Model for the Term Structure of Credit Spreads. Review of Financial Studies, 10, 481-523.

[20] Jorda, P.G. 2010. Modelling Credit Risk through Intensity Models. Working paper.

[21] Karatzas, I. y Shreve, S. 1988. Brownian Motion and Stochastic Calculus. SpringerVerlag N.Y.

[22] Karatzas, I. y Shreve, S. 1991. Stochastic Calculus for Finance II. Springer-Verlag N.Y. 
[23] Kim, Y.-J. y Kunitomo, N. 1999. Pricing options under stochastic interest rates: a new approach. Asia-Pacific Financial Markets 6, 49-70.

[24] Lando D. 2004. Credit risk modeling, Theory and Applications. Princeton University Press.

[25] Lamberton, D., Lapeyre, B. 2008. Introduction to Stochastic Calculus Applied to Finance. Chapman \& Hall, 2a edición.

[26] Longstaff, F., and E. Schwartz, 1995. Valuing Risky Debt: A New Approach. Journal of Finance, 50, 789-820.

[27] Longstaff, F. 1990. The Valuation of Options on Coupon Bonds. Unpublished Working Paper, The Ohio State University, Columbus.

[28] Longstaff, F. 1990. The Valuation of Options on Yields. Journal of Financial Economics, vol. 26, n. 1, pp. 97-121.

[29] Mendez M. 2010. Aplicaciones del cálculo estocástico al estudio de la estructura temporal de tasa de interés. Tesis Maestría. UAM.

[30] Merton, R.C. 1974. On the pricing of Corporate Debt: The Risk Structure of Interest Rates. The Journal of Finance, 29.

[31] Sukhomlin, N., Santana, L. 2010. Problema de calibración de mercado y estructura implícita del modelo de bonos de Black-Cox. Revista de métodos cuantitativos para la economía y la empresa (10). 73-98.

[32] Téllez, C. 2010. Medición del riesgo en crédito: Implementación y cálculo del VaR y el CVaR en tres modelos de incumplimiento. Tesis Maestría. UAM.

[33] Xie, D. 2008 Parametric Estimation for Treasury Bills. Journal of Finance and Economics. Issue 17.

[34] Yong-Jin Kim 2001 Option Pricing under Stochastic Interest Rates: An Empirical Investigation. Asia-Pacific Financial Markets. 9, 23-44. 
[35] Zhou, C., 1997. A Jump-Diffusion approach to Modeling Credit Risk and Valuing Defaultable Securities. Board of Governors of the Federal Reserve System Working Paper 97-15.

\section{Medios electrónicos}

[36] Abel Elizalde. http://www.abelelizalde.com. Última fecha de consulta: 20 de Septiembre 2012.

[37] Banxico. http://www.banxico.org.mx. Última fecha de consulta: 30 de Septiembre 2012.

[38] Bloomberg. http://www.bloomberg.com. Última fecha de consulta: 31 de Agosto 2012.

[39] Grupo BMV. http://www.bmv.com.mx. Última fecha de consulta: 30 de Septiembre 2012 .

[40] Moody's. http://www.moodys.com. Última fecha de consulta: 25 de Septiembre 2012

[41] Standard \& Poor's. http://www.standardandpoors.com. Última fecha de consulta: 25 de Septiembre 2012 